Ann. Scient. Éc. Norm. Sup.

$4{ }^{\text {e }}$ série, t. 48,2015 , p. 409 à 451

\title{
THE GEOMETRIC SATAKE CORRESPONDENCE FOR RAMIFIED GROUPS
}

\author{
BY XINWEN ZHU
}

Abstract. - We prove the geometric Satake isomorphism for a reductive group defined over $F=k((\mathrm{t}))$, and split over a tamely ramified extension. As an application, we give a description of the nearby cycles on certain Shimura varieties via the Rapoport-Zink-Pappas local models.

RÉSumÉ. - Nous démontrons l'isomorphisme de Satake géométrique pour un groupe réductif défini sur $F=k((\mathrm{t}))$ et déployé sur une extension modérément ramifiée. Nous donnons comme application une description des cycles évanescents sur certaines variétés de Shimura via les modèles locaux de Rapoport-Zink-Pappas.

\section{Introduction}

The Satake isomorphism (for unramified groups) is the starting point of the Langlands duality. Let us first recall its statement. Let $F$ be a non-Archimedean local field with ring of integers $\theta$ and residue field $k$, and let $G$ be a connected unramified reductive group over $F$ (e.g., $G=\mathrm{GL}_{n}$ ). Let $A \subset G$ be a maximal split torus of $G$, and $W_{0}$ be the Weyl group of $(G, A)$. Let $K$ be a hyperspecial subgroup of $G(F)$ containing $A(\theta)$ (e.g., $\left.K=\mathrm{GL}_{n}(\theta)\right)$. Then the classical Satake isomorphism describes the spherical Hecke algebra Sph $=C_{c}(K \backslash G(F) / K)$, the algebra of compactly supported bi- $K$-invariant functions on $G(F)$ under convolution. Namely, there is an isomorphism of algebras

$$
\mathrm{Sph} \simeq \mathbb{C}\left[\mathbb{X}_{\bullet}(A)\right]^{W_{0}},
$$

where $\mathbb{X}_{\bullet}(A)$ is the coweight lattice of $A$, and $\mathbb{C}\left[\mathbb{X}_{\bullet}(A)\right]^{W_{0}}$ denotes the $W_{0}$-invariants of the group algebra of $\mathbb{X}_{\bullet}(A)$.

If $F$ has positive characteristic $p>0$, then the classical Satake correspondence has a vast enhancement. For simplicity, let us assume that $G$ is split over $F$ (for the general case, see Theorem A.12). Let us write $G=H \otimes_{k} F$ for some split group $H$ over $k$ so that $K=H(\theta)$. Let $\mathrm{Gr}_{H}=H(F) / H(\theta)$ be the affine Grassmannian of $H$. Choose $\ell$ a prime different from $p$, 
and let $\operatorname{Sat}_{H}$ be the category of $(K \otimes \bar{k})$-equivariant perverse sheaves with $\overline{\mathbb{Q}}_{\ell}$-coefficients on $\mathrm{Gr}_{H} \otimes \vec{k}$. Then this is a Tannakian category and there is an equivalence

$$
\operatorname{Sat}_{H} \simeq \operatorname{Rep}\left(G \frac{\vee}{\mathbb{Q}_{\ell}}\right)
$$

where $G_{\mathbb{Q}_{\ell}}^{\vee}$ is the dual group of $G$ and $\operatorname{Rep}\left(G_{\overline{\mathbb{Q}}_{\ell}}^{\vee}\right)$ is the tensor category of algebraic representations of $G_{\overline{\mathbb{Q}}_{\ell}}^{\vee}$ (cf. $\left.[10,19]\right)$.

There is also a version of Satake isomorphism for an arbitrary reductive group over $F$, as recently proved by Haines and Rostami (cf. [12]) ${ }^{(1)}$. Namely, let $\mathcal{B}(G)$ be the Bruhat-Tits building of $G$ and $v \in \mathscr{B}(G)$ be a special vertex. Let $K_{v} \subset G(F)$ be the special parahoric subgroup of $G(F)$ corresponding to $v$. Let $A$ be a maximal split $F$-torus of $G$ such that $K_{v} \supset A(\theta)$, let $M$ be the centralizer of $A$ in $G$ and $W_{0}=N_{G}(A) / M$ be the Weyl group as before. Let $M_{1}$ be the unique parahoric subgroup of $M(F)$, and $\Lambda_{M}=M(F) / M_{1}$, which is a finitely generated Abelian group. Then

$$
C_{c}\left(K_{v} \backslash G(F) / K_{v}\right) \simeq \mathbb{C}\left[\Lambda_{M}\right]^{W_{0}} .
$$

More explicitly, suppose that $G$ is quasi-split so that $M=T$ is a maximal torus. Then

$$
\Lambda_{M}=\left(\mathbb{X}_{\bullet}(T)_{I}\right)^{\sigma},
$$

where $I$ is the inertial group and $\sigma$ is the Frobenius, and $\left(\mathbb{X}_{\bullet}(T)_{I}\right)^{\sigma}$ denotes the $\sigma$-invariants of the $I$-coinvariants of the group $\mathbb{X} .(T)$.

The goal of this paper is to provide a geometric version of the above isomorphism when $F$ has positive characteristic $p$ and the group $G$ is quasi-split and splits over a tamely ramified extension. More precisely, let $k$ be an algebraically closed field and let $\ell \neq \operatorname{char} k$ be a prime. Let $G$ be a group over the local field $F=k((\mathrm{t}))$ (so that $G$ is quasi-split automatically), which is split over a tamely ramified extension. That is, there is a finite extension $\tilde{F} / F$ such that $G_{\tilde{F}}$ is split and char $k \nmid[\tilde{F}: F]$. Let $v \in \mathcal{B}(G)$ be a special vertex in the building of $G$ and let $\underline{G}_{v}$ be the parahoric group scheme over $\theta=k[[t]]$ (in the sense of Bruhat-Tits), determined by $v$. We write $L G$ for the loop space of $G$ and $K_{v}=L^{+} \underline{G}_{v}$ for the jet space of $\underline{G}_{v}$. By definition, for any $k$-algebra $R, L G(R)=G\left(R \hat{\otimes}_{k} F\right)$ and $K_{v}(R)=\underline{G}_{v}\left(R \hat{\otimes}_{k} \theta\right)$. Let

$$
\mathcal{F} \ell_{v}=L G / K_{v}
$$

be the (twisted) affine flag variety ${ }^{(2)}$, which is an ind-scheme over $k$. Let $\mathscr{P}_{v}=\mathscr{P}_{K_{v}}\left(\mathcal{F} \ell_{v}\right)$ be the category of $K_{v}$-equivariant perverse sheaf on $\mathcal{F} \ell_{v}$, with coefficients in $\overline{\mathbb{Q}}_{\ell}$. Let $H$ be a split Chevalley group over $\mathbb{Z}$ such that $G \otimes_{F} F^{s} \simeq H \otimes F^{s}$, where $F^{s}$ is a (fixed) separable closure of $F$. Then there is a natural action of $I=\operatorname{Gal}\left(F^{s} / F\right)$ on $H^{\vee}:=H_{\mathbb{Q}_{\ell}}^{\vee}$ (preserving a fixed pinning).

Theorem 0.1. - The category $\mathscr{P}_{v}$ has a natural tensor structure. In addition, as tensor categories, there is an equivalence

$$
\mathscr{R} \varnothing: \operatorname{Rep}\left(\left(H^{\vee}\right)^{I}\right) \simeq \mathscr{P}_{v},
$$

(1) There is another version, known earlier, as in [6].

(2) One would call $\mathcal{G} \ell_{v}$ the affine Grassmannian of $G$. However, we reserve the name "affine Grassmannian" of $G$ for another object, as defined in Definition A.2.

4 e SÉRIE - TOME $48-2015$ - No 2 
such that $\mathrm{H}^{*} \circ \mathscr{R} \&$ is isomorphic to the forgetful functor, where $\mathrm{H}^{*}$ is the hypercohomology functor.

This theorem can be regarded as a categorification of $(0.1)$ in the case when $k$ is algebraically closed and the group splits over a tamely ramified extension of $k((\mathrm{t}))$. For the description of $\left(H^{\vee}\right)^{I}$ when $H$ is absolutely simple and simply-connected, see $\S 4$.

Let us point out the following remarkable facts when the group is ramified. First, the group $\left(H^{\vee}\right)^{I}$ is not necessarily connected as is shown in Remark (4.4). Second, it is wellknown that if $G$ is unramified over $F$, then all the hyperspecial subgroups of $G$ are conjugate under $G_{\text {ad }}(F)([27, \S 2.5])$, where $G_{\text {ad }}$ is the adjoint group of $G$. However, this is no longer true for special parahoric of $G$ if $G$ is ramified. An example is given by the odd ramified unitary similitude group $\mathrm{GU}_{2 m+1}$. There are essentially two types of special parahorics of $\mathrm{GU}_{2 m+1}$, as given in (7.1). One of them has reductive quotient $\mathrm{GO}_{2 m+1}$ (denoted by $\underline{G}_{v_{0}}$ ), and the other has reductive quotient $\mathrm{GSp}_{2 m}$ (denoted $\underline{G}_{v_{1}}$ ). Accordingly, the geometry of the corresponding flag varieties $\mathcal{F} \ell_{v_{0}}$ and $\mathcal{F} \ell_{v_{1}}$ are very different, while $\mathscr{P}_{v_{0}} \simeq \mathscr{P}_{v_{1}}$. Indeed, their Schubert varieties (i.e., closures of $K_{v_{i}}$-orbits) are both parameterized by irreducible representations of $\mathrm{GO}_{2 m+1}$. Let $\mathcal{F} \ell_{v_{0} \bar{\mu}_{2 m, 1}}$ (resp. $\mathcal{F} \ell_{v_{1} \bar{\mu}_{2 m, 1}}$ ) be the Schubert variety in $\mathcal{F} \ell_{v_{0}}$ (resp. $\mathcal{F} \ell_{v_{1}}$ ) parameterized by the standard representation of $\mathrm{GO}_{2 m+1}$. Then it is shown in [31] that $\mathcal{F} \ell_{v_{0} \bar{\mu}_{2 m, 1}}$ is not Gorenstein, while in [25] that $\mathcal{F} \ell_{v_{1} \bar{\mu}_{2 m, 1}}$ is smooth. On the other hand, the intersection cohomology of both varieties gives the standard representation of $\mathrm{GO}_{2 m+1}$. In addition, the stalk cohomologies of both sheaves are the "same". See Theorem 0.3 below.

REMARK 0.1. - Instead of considering a special parahoric $K_{v}$ of $L G$, one can begin with the special maximal "compact" $K_{v}^{\prime}$, (i.e., $K_{v}^{\prime}=L^{+} \underline{G}_{v}^{\prime}$, where $\underline{G}_{v}^{\prime}$ is the stabilizer group scheme of $v$ as constructed by Bruhat-Tits), and consider the category of $K_{v}^{\prime}$-equivariant perverse sheaves on $L G / K_{v}^{\prime}$. However, from a geometric point of view, this is less natural since $K_{v}^{\prime}$ is not necessarily connected and the category of $K_{v}^{\prime}$-equivariant perverse sheaves is complicated. In fact, we do not know how to relate this category to the Langlands dual group yet. In addition, when we discuss the Langlands parameters in Section 6, it is also more "correct" to consider $K_{v}$ rather than $K_{v}^{\prime}$.

The idea of the proof of the theorem is as follows. Using Gaitsgory's nearby cycle functor construction as in $[8,31]$, we construct a functor

$$
\mathcal{Z}: \operatorname{Sat}_{H} \rightarrow \mathscr{P}_{v}
$$

which is a central functor in the sense of [2]. By standard arguments in the theory of Tannakian equivalence and the Mirkovic-Vilonen theorem, this already implies that $\mathscr{P}_{v} \simeq \operatorname{Rep}\left(\tilde{G}^{\vee}\right)$ for certain closed subgroup $\tilde{G}^{\vee} \subset H^{\vee}$. Then we identify $\tilde{G}^{\vee}$ with $\left(H^{\vee}\right)^{I}$ using the parametrization of the $K_{v}$-orbits on $\mathcal{F} \ell_{v}$.

REMARK 0.2. - (i) We believe that the same argument (maybe with small modifications) should work for groups split over wild ramified extensions. However, we have not checked this carefully.

(ii) Our approach is more inspired by [8] rather than [19]. However, it would be interesting to know whether there is the similar theory of MV-cycles in the ramified case. It seems that 
the geometry of semi-infinite orbits on $\mathcal{F} \ell_{v}$ is similar to the unramified case, except when $\mathcal{F} \ell_{v}$ corresponds to one type of special parahorics for odd unitary groups (the one denoted by $\underline{G}_{v_{1}}$ as above). We do not know what happens in this last case.

(iii) Our theorem and the method also share the similar features with the results of Nadler on geometric Satake for real groups [20].

When the group $G$ is quasi-split over the non-Archimedean local field $F=\mathbb{F}_{q}((\mathrm{t}))$ and $v$ is a special vertex of $\mathcal{B}(G, F)$, the affine flag variety $\mathcal{F} \ell_{v}$ is defined over $\mathbb{F}_{q}$. We assume that $v$ is very special, i.e., it remains special when we base change $G$ to $\overline{\mathbb{F}}_{q}((\mathrm{t}))$ (see $\S 6$ for more discussions of this notion). Then we can consider the category of $K_{v}$-equivariant semi-simple perverse sheaves on $\mathcal{F} \ell_{v}$, pure of weight zero, and denote it by $\mathscr{P}_{v}^{0}$. On the other hand, let $I$ be the inertial group of $F$ and $\sigma$ be the Frobenius of $\operatorname{Gal}\left(k / \mathbb{F}_{q}\right)$, where $k=\overline{\mathbb{F}}_{q}$. Then the action of $\operatorname{Gal}\left(F^{s} / F\right)$ on $H^{\vee}$ (via the pinned automorphisms) induces a canonical action of $\sigma$ on $\left(H^{\vee}\right)^{I}$, denoted by act ${ }^{\text {alg }}$. One can form the semidirect product $\left(H^{\vee}\right)^{I} \rtimes_{\text {actalg }} \operatorname{Gal}\left(k / \mathbb{F}_{q}\right)$, which can be regarded as a proalgebraic group over $\overline{\mathbb{Q}}_{\ell}$, and consider the category of algebraic representations of $\left(H^{\vee}\right)^{I} \rtimes_{\text {act }}{ }^{\text {alg }} \operatorname{Gal}\left(k / \mathbb{F}_{q}\right)$, denoted by $\operatorname{Rep}\left(\left(H^{\vee}\right)^{I} \rtimes_{\text {act }}\right.$ alg $\left.\operatorname{Gal}\left(k / \mathbb{F}_{q}\right)\right)$.

Theorem 0.2. - In this case, the functor $\mathcal{R} \phi$ in Theorem 0.1 can be extended to an equivalence

$$
\Re \varnothing: \operatorname{Rep}\left(\left(H^{\vee}\right)^{I} \rtimes_{\text {actalg }} \operatorname{Gal}\left(k / \mathbb{F}_{q}\right)\right) \simeq \mathscr{P}_{v}^{0},
$$

whose composition with $\mathrm{H}^{*}$ is isomorphic to the forgetful functor.

Let us mention that under this equivalence, the restriction to $\operatorname{Gal}\left(k / \mathbb{F}_{q}\right)$ of the representation $\left(H^{\vee}\right)^{I} \rtimes_{\text {actalg }} \operatorname{Gal}\left(k / \mathbb{F}_{q}\right)$ on $\mathrm{H}^{*}(\mathcal{F})$ for $\mathcal{F} \in \mathscr{D}_{v}^{0}$ is NOT the natural Galois action of $\operatorname{Gal}\left(k / \mathbb{F}_{q}\right)$ on $\mathrm{H}^{*}(\mathcal{F})$. However, their difference can be described explicitly. See Section 4 and appendix for more details.

Our next result is to use the ramified geometric Satake isomorphism to obtain the stalk cohomology of sheaves on $\mathcal{F} \ell_{v}$ (i.e., the corresponding Lusztig-Kato polynomial in ramified case), following an idea of Ginzburg (cf. [10]). Let us state the result precisely. The centralizer of $A$ in our case is a maximal torus of $G$, denoted by $T$. Then the $K$-orbits on $\mathcal{F} \ell_{v}$ are labeled by $\mathbb{X}_{\bullet}(T)_{I} / W_{0}, W_{0}$-orbits of the coinvariants of the cocharacter group of $T$. For $\bar{\mu} \in \mathbb{X}_{\bullet}(T)_{I}$, let $\dot{\mathcal{F}} \ell_{v \bar{\mu}}$ be the corresponding orbit. For a representation $V$ of $\left(H^{\vee}\right)^{I}$, let $V(\bar{\mu})$ be the weight space of $V$ for $\left(T^{\vee}\right)^{I}$. Let $X^{\vee} \in \operatorname{Lie}\left(H^{\vee}\right)^{I}$ be a certain principal nilpotent element (see Section 5 for the details), which induces a filtration $F_{i} V(\bar{\mu})=\left(\operatorname{ker} X^{\vee}\right)^{i+1} \cap V(\bar{\mu})$ on $V(\bar{\mu})$, called the Brylinski-Kostant filtration. Then we have

Theorem 0.3. - For $V \in \operatorname{Rep}\left(\left(H^{\vee}\right)^{I}\right)$, let $\mathscr{R} \phi(V) \in \mathscr{D}_{v}$ be the corresponding sheaf. Then

$$
\left.\operatorname{dim} \mathcal{H}^{2 i-(2 \rho, \bar{\mu})} \mathscr{R} \&(V)\right|_{\mathcal{F} \ell_{v \bar{\mu}}}=\operatorname{dim}_{\operatorname{gr}_{i}^{F}} V(\bar{\mu}) .
$$

Here $\mathcal{H}^{*}$ denotes the cohomology sheaves, and $2 \rho$ is the sum of positive roots of $H$, see Section 1 for the meaning of $(2 \rho, \bar{\mu})$.

One of our main motivations of this work is to apply these results to the calculation of the nearby cycles of certain ramified unitary Shimura varieties, via the Rapoport-Zink-Pappas local models. For example, we obtain the following theorem (see Section 7 for details). 
TheOrem 0.4. - Let $G=\mathrm{GU}(r, s)$ be a unitary similitude group associated to an imaginary quadratic extension $F / \mathbb{Q}$ and a Hermitian space $(W, \phi)$ over $F / \mathbb{Q}$. Let $p>2$ be a prime where $F / \mathbb{Q}$ is ramified and the Hermitian form is split. Let $K_{p}$ be a special parahoric subgroup of $G=G\left(\mathbb{Q}_{p}\right)$. Let $K=K_{p} K^{p} \subset G\left(\mathbb{Q}_{p}\right) G\left(\mathbb{A}_{f}^{p}\right)$ be a compact open subgroup with $K^{p}$ small enough. Let $\mathrm{Sh}_{K}$ be the associated Shimura variety over the reflex field $E$ and $\mathrm{Sh}_{K_{p}}$ be the integral model of $\mathrm{Sh}_{K}$ over $\theta_{E_{p}}$ (as defined in [23]). Then for $\ell \neq p$, the action of the inertial subgroup I of $\operatorname{Gal}\left(\overline{\mathbb{Q}}_{p} / F_{p}\right)$ on the nearby cycle $\Psi_{\mathrm{Sh}_{K_{p}} \otimes_{\vartheta_{E_{p}}} \vartheta_{F_{p}}}\left(\mathbb{Q}_{\ell}\right)$ is trivial.

By applying Theorem 0.3, it will not be hard to determine the traces of Frobenius on these sheaves explicitly, which will be the input of the Langlands-Kottwitz method to determine the local Zeta factors of $\mathrm{Sh}_{K}$. Instead, we characterize these traces of Frobenius in terms of Langlands parameters, which verifies a conjecture of Haines and Kottwitz in this case (see Proposition 7.4).

Remark 0.3. - (i) While the definition of the integral model of a PEL-type Shimura variety at an "unramified" prime $p$ (i.e., the group is unramified at $p$ and $K_{p}$ is hyperspecial) is well-known (cf. [15]), the definition of such a model at the ramified prime $p$ (even for $K_{p}$ special) is a subtle issue. In [21, 23], the integral models $\mathrm{Sh}_{K_{p}}$ are defined as certain closed subschemes of certain moduli problems of Abelian varieties. Except a few cases (e.g., $(r, s)=(n-1,1)$ and $n=r+s$ is small), there is no moduli description of $\mathrm{Sh}_{K_{p}}$ so far. In general, $\mathrm{Sh}_{K_{p}}$ are not smooth. Indeed, as shown in [21, 23], when $n=r+s$ is odd and $(r, s)=(n-1,1)$, for the special parahoric $K_{p}$ of $G\left(\mathbb{Q}_{p}\right)$ with reductive quotient $\mathrm{GO}_{n}, \mathrm{Sh}_{K_{p}}$ is not even semi-stable.

(ii) If $r \neq s$, then we know that $E=F$ and the above theorem gives a complete description of the monodromy on the nearby cycles of $\mathrm{Sh}_{K_{p}}$. If $r=s$, then $E=\mathbb{Q}$, and the complete description of the monodromy is more complicated. See Section 7 for details. In any case, the action of inertia on the nearby cycle is semi-simple.

(iii) We hope that there will be a "good" compactification of such Shimura varieties $\mathrm{Sh}_{K_{p}}$. Then the above theorem, together with the existence of such compactification, would imply that the monodromy of $H_{c}^{*}\left(\mathrm{Sh}_{K} \otimes_{E_{p}} F_{p}\right)$ is trivial.

(iv) The triviality of the monodromy as above would have the following surprising consequence for the Albanese of Picard modular surfaces. Namely, in the case when $(r, s)=(2,1)$, $F / \mathbb{Q}$ is ramified at $p>2$ and $K_{p}=G\left(\mathbb{Q}_{p}\right)$ is a special parahoric, the Albanese $\operatorname{Alb}\left(\mathrm{Sh}_{K_{p}}\right)$ of $\mathrm{Sh}_{K_{p}}$ is trivial. It will be interesting to find the "optimal" level structure at $p$ so that $\mathrm{Alb}\left(\mathrm{Sh}_{K_{p}}\right)$ can be possibly non-trivial. More detailed discussion will appear elsewhere.

Let us quickly describe the organization of the paper. We will prove Theorem 0.1 and Theorem 0.2 in $\S 1-4$. Then we prove Theorem 0.3 in $\S 5$.

In $\S 6$, we briefly discuss the Langlands parameters associated to a smooth representation of a quasi-split $p$-adic group, which has a vector fixed by a special parahoric. We call them "spherical" representations, and we will see that their Langlands parameters can be described easily. Again, the correct point of view is to consider the special parahoric rather than the special maximal compact. Then in $\S 7$, we apply the previous results to study the nearby cycles on certain unitary Shimura varieties. 
The paper contains an appendix, joint with T. Richarz, where we recover the full Langlands dual group via the Tannakian formalism. In particular, we give the geometric Satake correspondence for unramified groups. We hope that this formulation will be of independent interest. In addition, we observe that for a reductive group defined over $k$, the Tannakian formalism provides a natural action act ${ }^{\text {geom }}$ of $\operatorname{Gal}\left(k^{s} / k\right)$ on the dual group $G^{\vee}$, which differs from the usual pinned action act ${ }^{\text {alg }}$ of $\operatorname{Gal}\left(k^{s} / k\right)$ on $G^{\vee}$ by the twist of "the half sum of the positive roots". This gives a geometric explanation of the two natural normalizations of the Satake parameters.

Notations. - Let $k$ be a field. We denote by $k^{s}$ a separable closure of $k$.

For a (not necessarily connected) diagonalizable algebraic group $C$ defined over a field $F$, we denote by $\mathbb{X}^{\bullet}(C)$ its group of characters and by $\mathbb{X}_{\bullet}(C)$ its group of cocharacters over the separable closure $F^{s}$. The Galois group $I=\operatorname{Gal}\left(F^{s} / F\right)$ acts on $\mathbb{X}^{\bullet}(C)\left(\right.$ resp. $\left.\mathbb{X}_{\bullet}(C)\right)$ and the invariants (resp. coinvariants) are denoted by $\mathbb{X}^{\bullet}(C)^{I}$ (resp. $\left.\mathbb{X}^{\bullet}(C)_{I}, \mathbb{X}_{\bullet}(C)^{I}, \mathbb{X}_{\bullet}(C)_{I}\right)$. We will always use $\lambda, \mu, \ldots$ to denote elements in $\mathbb{X}^{\bullet}(C)$ or $\mathbb{X}_{\bullet}(C)$ and $\bar{\lambda}, \bar{\mu}$ to denote elements in $\mathbb{X}^{\bullet}(C)_{I}$ or $\mathbb{X}_{\bullet}(C)_{I}$. In general, let $I$ be a group acting on a set $S$. We denote $S^{I}$ to be the subset of fixed points.

If $G$ is an algebraic group defined over a field $E$, we denote by $\operatorname{Rep}(G)$ the category of finite dimensional representations of $G$ over $E$. If $G$ is connected reductive, we denote $G_{\mathrm{ad}}, G_{\mathrm{der}}, G_{\mathrm{sc}}$ to be its adjoint quotient, its derived group, and the simply-connected cover of its derived group.

Let $k$ be a field and $\theta=k[[t]], F=k((\mathrm{t})):=k[[t]]\left[t^{-1}\right]$. For an $\theta$-scheme $X$, we denote $L^{+} X$ to be the jet space over $k$ so that for any $k$-algebra $R, L^{+} X(R)=X(R[[t]])$. For an $F$-scheme $X$, we denote $L X$ to be its loop space so that $L X(R)=X(R((\mathrm{t})))$. If $X$ is defined over $k$, we write $L^{+} X$ for $L^{+}(X \otimes \theta)$ and $L X$ for $L(X \otimes F)$ if no confusion will arise.

For a variety $X$ over $k$, we denote $D(X)$ the usual (bounded) derived category of $\ell$-adic sheaves on $X(\ell \nmid \operatorname{char} k)$. If $X=\lim X_{i}$ is an ind-scheme of ind-finite type, $D(X)=\lim D\left(X_{i}\right)$ as usual. If there is an action of an algebraic group $G$ on $X$, the $G$-equivariant derived category is denoted by $D_{G}(X)$ (see [1] for the details). All the functors like $f_{*}, f_{!}, f^{*}, f^{!}$are understood in the derived sense unless otherwise specified.

Acknowledgement. - The author would like to thank D. Gaitsgory, T. Haines, Y. Liu, I. Mirković, G. Pappas, M. Rapoport, T. Richarz, E. Urban, Z. Yun for useful discussions. The author also thanks the hospitality of Tsinghua University, where part of the work has been done. The work of the author is supported by the NSF grant under DMS-1001280.

\section{Reminders on the affine flag variety associated to a special parahoric}

In this section, we collect basic facts about the affine flag varieties associated to a special parahoric of $G$. Another purpose of this section is to fix notations that are used in the later sections.

Let $k$ be an algebraically closed field and let $G$ be a group over the local field $F=k((\mathrm{t}))$, which is split over a tamely ramified extension. Let us choose $A$ to be a maximal $F$-split torus

4 e SÉRIE - TOME $48-2015$ - No 2 
of $G$ and $T$ be its centralizer. Then $T$ is a maximal torus of $G$ since $G$ is quasi-split. Let us choose a rational Borel subgroup $B \supset T$.

Let $H$ be a split Chevalley group over $\mathbb{Z}$ such that $H \otimes F^{s} \simeq G \otimes F^{s}$. We need to choose this isomorphism carefully. Let us fix a pinning $\left(H, B_{H}, T_{H}, X\right)$ of $H$ over $\mathbb{Z}$. Let us recall that this means that $B_{H}$ is a Borel subgroup of $H, T_{H}$ is a split maximal torus contained in $B_{H}$, and $X=\Sigma_{\tilde{a} \in \Delta_{H}} X_{\tilde{a}} \in \operatorname{Lie} B$, where $\Delta_{H}$ is the set of simple roots of $H, \tilde{U}_{\tilde{a}}$ is the root subgroup corresponding to $\tilde{a}$ and $X_{\tilde{a}}$ is a generator in the rank one free $\mathbb{Z}$-module Lie $\tilde{U}_{\tilde{a}}$. Let $\Xi$ be the group of pinned automorphisms of $\left(H, B_{H}, T_{H}, X\right)$, which is canonically isomorphic to the group of the automorphisms of the root datum $\left(\mathbb{X}^{\bullet}\left(T_{H}\right), \Delta_{H}, \mathbb{X}_{\bullet}\left(T_{H}\right), \Delta_{H}^{\vee}\right)$.

Let us choose an isomorphism $(G, B, T) \otimes_{F} \tilde{F} \simeq\left(H, B_{H}, T_{H}\right) \otimes_{\mathbb{Z}} \tilde{F}$, where $\tilde{F} / F$ is a cyclic extension such that $G \otimes \tilde{F}$ splits. This induces an isomorphism of the root data $\left(\mathbb{X}^{\bullet}\left(T_{H}\right), \Delta_{H}, \mathbb{X}_{\bullet}\left(T_{H}\right), \Delta_{H}^{\vee}\right) \simeq\left(\mathbb{X} \bullet(T), \Delta, \mathbb{X}_{\bullet}(T), \Delta^{\vee}\right)$. Now the action of $I=\operatorname{Gal}(\tilde{F} / F)$ on $G \otimes_{F} \tilde{F}$ induces a homomorphism $\psi: I \rightarrow \Xi$. Then we can always choose an isomorphism

$$
(G, B, T) \otimes_{F} \tilde{F} \simeq\left(H, B_{H}, T_{H}\right) \otimes_{\mathbb{Z}} \tilde{F}
$$

such that the action of $\gamma \in I$ on the left hand side corresponds to $\psi(\gamma) \otimes \gamma$. In the rest of the paper, we fix such an isomorphism.

Recall that the Kottwitz homomorphism $\kappa: T(F) \rightarrow \mathbb{X}_{\bullet}(T)_{I}$ (cf. $[16,11]$ ) induces an isomorphism

$$
\mathbb{X}_{\bullet}(T)_{I} \simeq T(F) / \underline{T}^{b, 0}(\theta),
$$

where $\underline{T}^{b, 0}$ is the unique parahoric group scheme of $T$ over $\theta$ (the connected Néron model). Our convention of Kottwitz homomorphism is that the action of $t \in T(F)$ on $A(G, A)$ (the apartment associated to $(G, A))$ is given by $v \mapsto v-\kappa(\mathrm{t})$. Let $W_{0}=W(G, A)$ be the relative Weyl group of $G$. It acts on $T$ and therefore on $\mathbb{X}_{\bullet}(T)_{I}$. In addition, its action on the torsion subgroup $\mathbb{X}_{\bullet}(T)_{I, \text { tor }} \subset \mathbb{X}_{\bullet}(T)_{I}$ is trivial.

The Borel subgroup $B$ determines a set of positive roots $\Phi^{+}=\Phi(G, A)^{+}$for $G$. There is a natural map $\mathbb{X}_{\bullet}(T)_{I} \rightarrow \mathbb{X}_{\bullet}(T)_{I} \otimes \mathbb{R} \simeq \mathbb{X}_{\bullet}(A)_{\mathbb{R}}$. We define the set of dominant elements in $\mathbb{X}_{\bullet}(T)_{I}$ to be

$$
\mathbb{X}_{\bullet}(T)_{I}^{+}=\left\{\bar{\mu} \mid(\bar{\mu}, a) \geq 0 \text { for } a \in \Phi^{+}\right\} .
$$

Then the natural map $\mathbb{X}_{\bullet}(T)_{I}^{+} \subset \mathbb{X}_{\bullet}(T)_{I} \rightarrow \mathbb{X}_{\bullet}(T)_{I} / W_{0}$ is bijective. Let us define an order $\preceq$ on $\mathbb{X}_{\bullet}(T)_{I}$ as follows. Let $Q_{H}$ be the coroot lattice for $H$. The action of $I$ on $Q_{H}$ will send the positive coroots of $H$ (determined by the chosen Borel) to positive coroots. Therefore, it makes sense to talk about positive elements in $\left(Q_{H}\right)_{I}$. Namely, an element in $\left(Q_{H}\right)_{I}$ is positive if its preimage in $Q_{H}$ is a sum of positive coroots (of $H$ ). Since $\left(Q_{H}\right)_{I} \subset \mathbb{X} \bullet(T)_{I}$, we can define for $\lambda, \mu \in \mathbb{X} \bullet(T)_{I}$,

$$
\bar{\lambda} \preceq \bar{\mu} \text { if } \bar{\mu}-\bar{\lambda} \text { is positive in }\left(Q_{H}\right)_{I} .
$$

Let $\underline{G}_{v}$ be a special parahoric group scheme of $G$ over $\theta=k[[t]]$ in the sense of BruhatTits (see [27] for a summary of the theory), such that the natural inclusion $A \subset G$ extends to $A_{\vartheta} \subset \underline{G}_{v}$ (i.e., the vertex $v$ in the (reduced) building of $G$ corresponding to $\underline{G}_{v}$ is contained in the apartment $A(G, A)$. For examples of such group schemes, we refer to Section 7. We write $K_{v}=L^{+} \underline{G}_{v}$, and consider the (twisted) affine flag variety

$$
\mathcal{F} \ell_{v}=L G / K_{v}
$$


This is an ind-projective scheme (cf. [22]). As is shown in loc. cit., when $G$ is semi-simple and simply-connected, $\mathcal{F} \ell_{v}$ is just a partial flag variety of certain (twisted) affine Kac-Moody group. The $K_{v}$-orbits on $\mathcal{F} \ell_{v}$ are parameterized by $\mathbb{X}_{\bullet}(T)_{I}^{+}$. For $\bar{\mu} \in \mathbb{X}_{\bullet}(T)_{I}^{+}$, let $s_{\bar{\mu}}$ denote the point in $\mathcal{F} \ell_{v}$ corresponding to $\bar{\mu}$. More precisely, this point is the image of $\bar{\mu}$ under the $\operatorname{map} \mathbb{X}_{\bullet}(T)_{I} \simeq T(F) / \underline{T}^{b, 0}(\theta) \rightarrow G(F) / K_{v}=\mathcal{F} \ell_{v}(\mathrm{k})$. Let $\mathcal{F} \ell_{v \bar{\mu}}$ be the corresponding Schubert variety, i.e., the closure of $K$-orbit through $s_{\bar{\mu}}$. Then

$$
\operatorname{dim} \mathcal{F} \ell_{v \bar{\mu}}=(2 \rho, \bar{\mu}),
$$

where $2 \rho$ is the sum of all positive roots (for $H$ ), and by definition $(2 \rho, \bar{\mu})=(2 \rho, \mu)$ for any lift $\mu$ of $\bar{\mu}$ to $\mathbb{X}_{\bullet}(T)$. In addition, $\mathscr{F} \ell_{v \bar{\lambda}} \subset \mathcal{F} \ell_{v \bar{\mu}}$ if and only if $\bar{\lambda} \preceq \bar{\mu}$. In this case, $\operatorname{dim} \mathcal{F} \ell_{v \bar{\mu}}-\operatorname{dim} \mathcal{F} \ell_{v \bar{\lambda}}$ is an even integer. For the proof of these facts, see [25, 31].

Let $\mathscr{P}_{v}=\mathscr{P}_{K_{v}}\left(\mathcal{F} \ell_{v}\right)$ be the category of $K_{v}$-equivariant perverse sheaves on $\mathcal{F} \ell_{v}$, with coefficients in $\overline{\mathbb{Q}}_{\ell}$. By the above facts, in each connected component of $\mathcal{F} \ell_{v}$, the dimensions of $K_{v}$-orbits have the constant parity. Therefore, we have

Lemma 1.1. - $\mathscr{P}_{v}$ is a semi-simple Abelian category.

Proof. - By the argument as in [8, Proposition 1], it is enough to show that the stalks of the intersection cohomology sheaves have the parity vanishing property. But this follows from the existence of Demazure resolutions of Schubert varieties in $\mathcal{F} \ell_{v}$ whose fibers have pavings by affine spaces (for example see [8, A.7]). More precisely, the existence of such resolutions were constructed in [22, Section 8] for twisted affine flag varieties, and the arguments as in [13] apply in this situation to show that the fibers have pavings by affine spaces.

In [31, 24], a natural $\mathbb{G}_{m}$-action on $\mathcal{F} \ell_{v}$ is constructed. In the Kac-Moody setting, it is just the action of the "rotation torus" on $\mathcal{F} \ell_{v}$. Each Schubert cell is invariant under this action.

Corollary 1.2. - Any $K_{v}$-equivariant perverse sheaf on $\mathcal{F} \ell_{v}$ is automatically $\mathbb{G}_{m}$-equivariant.

Proof. - Clearly, the intersection complex is $\mathbb{G}_{m}$-equivariant. Then the assertion follows from the semisimplicity of $\mathscr{P}_{v}$.

EXAmPLE 1.3. - In the special case when $G=H \otimes_{k} F$ and $\underline{G}_{v}=H \otimes_{k} \theta$ is hyperspecial, then $\mathcal{F} \ell_{v}$ is just the usual affine Grassmannian $\mathrm{Gr}_{H}$ of $H$, and $\mathscr{P}_{v}$ is the Satake category of $H$, i.e., the category of $L^{+} H$-equivariant perverse sheaves on $\mathrm{Gr}_{H}$. All the above facts are well-known.

\section{Construction of the functor $Z$}

We continue the notations as in the previous sections and let $\underline{G}_{v}$ be a special parahoric group scheme of $G$ over $\mathscr{\theta}$. In [31], a group scheme $\mathscr{G}$ over $\mathbb{A}_{k}^{1}$ is constructed such that

1. $\mathscr{G}_{\eta}$ is connected reductive, splits over a (tamely) ramified extension, where $\eta$ is the generic point of $\mathbb{A}_{k}^{1}$;

2. For some choice of isomorphism $F_{0} \simeq F, \mathscr{G}_{F_{0}} \simeq G$, where for a point $x \in \mathbb{A}_{k}^{1}$, $\theta_{x}$ denotes the completed local ring at $x$ and $F_{x}$ denotes the fractional field of $\theta_{x}$;

3. For any $y \neq 0, \mathscr{G}_{\vartheta_{y}}$ is hyperspecial, (non-canonically) isomorphic to $H \otimes \vartheta_{y}$; 
4. $\mathscr{G}_{\vartheta_{0}}=\underline{G}_{v}$ under the isomorphism $\mathscr{G}_{F_{0}} \simeq G$.

The construction is as follows. Regard $I$ as the Galois group of the cyclic cover $[e]: \mathbb{G}_{m} \rightarrow \mathbb{G}_{m}$ of degree $e$. Then the group $I$ acts $H \times \mathbb{G}_{m}$. Namely, it acts on $H$ via the pinned automorphism $\psi: I \rightarrow \Xi$, and on $\mathbb{G}_{m}$ via transport of structures. Then $\left.\mathscr{G}\right|_{\mathbb{G}_{m}}=\left(\operatorname{Res}_{\mathbb{G}_{m} / \mathbb{G}_{m}}\left(H \times \mathbb{G}_{m}\right)\right)^{I}$, and $\mathscr{G}$ is the extension of it to $\mathbb{A}^{1}$ so that $\mathscr{G}_{\vartheta_{0}}=\underline{G}_{v}$.

Let $\mathrm{Gr}_{\mathscr{g}}$ be the global affine Grassmannian of $\mathscr{G}$, which is an ind-scheme over $\mathbb{A}^{1}$ (see, for example $[24, \S 5]$ for the ind-representability of $\left.\operatorname{Gr}_{\mathscr{g}}\right)$. Recall that it classifies triples $(y, \mathcal{E}, \beta)$ where $y$ is a point on $\mathbb{A}_{k}^{1}, \mathcal{E}$ is a $\mathscr{G}$-torsor on $\mathbb{A}^{1}$ and $\beta$ is a trivialization of this $\mathscr{G}$-torsor away from $y$. Let $[e]: \mathbb{A}^{1} \rightarrow \mathbb{A}^{1}$ be the natural extension of the cyclic cover of $[e]: \mathbb{G}_{m} \rightarrow \mathbb{G}_{m}$. Let $\widetilde{\operatorname{Gr}}_{g}:=\operatorname{Gr}_{g} \times \mathbb{A}^{1},[e] \mathbb{A}^{1}$ be the base change. Then

$$
\left(\widetilde{\mathrm{Gr}}_{\mathscr{G}}\right)_{0} \simeq \mathcal{F} \ell_{v},\left.\quad \widetilde{\mathrm{Gr}}_{\mathscr{g}}\right|_{\mathbb{G}_{m}} \simeq \mathrm{Gr}_{H} \times \mathbb{G}_{m} .
$$

Since $I$ acts on $H$ via pinned automorphisms, it acts on $\mathrm{Gr}_{H}$, still denoted by $\psi$. The following lemma is clear from the above construction.

Lemma 2.1. - Under the isomorphism $\mathrm{Gr}_{g} \times_{\mathbb{G}_{m}} \mathbb{G}_{m} \simeq \mathrm{Gr}_{H} \times \mathbb{G}_{m}$, the action of $\gamma \in I$ on the left hand side (via the Galois action on the second factor) corresponds to the action of $\psi(\gamma) \times \gamma$ on the right hand side.

REMARK 2.2. - One should be warned that $\widetilde{\mathrm{Gr}}_{\mathscr{G}} \neq \mathrm{Gr}_{\tilde{\mathscr{G}}}$, where $\widetilde{\mathscr{G}}$ is the base change of $\mathscr{G}$ along $[e]: \mathbb{A}^{1} \rightarrow \mathbb{A}^{1}$.

Recall that we denote $\operatorname{Sat}_{H}$ to be the Satake category for $H$, i.e., the category of $L^{+} H$-equivariant perverse sheaves on $\operatorname{Gr}_{H}$, which is equivalent to $\operatorname{Rep}\left(H^{\vee}\right)$ via the geometric Satake correspondence. Let

$$
8: \operatorname{Rep}\left(H^{\vee}\right) \rightarrow \operatorname{Sat}_{H}
$$

be this equivalence. We define a functor

$$
\mathcal{Z}: \operatorname{Sat}_{H} \rightarrow \mathscr{P}_{v}
$$

by taking the nearby cycles. More precisely, let

$$
Z(\mathcal{F})=\Psi_{\widetilde{\mathrm{Gr}} \mathscr{g}}\left(\mathcal{F} \otimes \overline{\mathbb{Q}}_{\ell}[1]\right)
$$

where for an (ind)-scheme $\chi$ of (ind)-finite type over $\mathbb{A}^{1}, \Psi_{\chi}$ denotes the usual nearby cycle functor (see SGA 7, XIII for the definition of nearby cycles, and [8, A.2] for the explanation why the nearby cycles functors extend to ind-schemes of ind-finite type). Recall that the theory of nearby cycles provides an action of $\operatorname{Gal}\left(F^{s} / F\right)$ on the functor $Z$ via automorphisms, usually called the monodromy action.

Lemma 2.3. - The monodromy of $\mathcal{Z}(\mathcal{F})$ is trivial.

Proof. - This follows from the fact that there is a $\mathbb{G}_{m}$-action on $\widetilde{\mathrm{Gr}}_{\mathscr{g}}$ making the natural map $\widetilde{\mathrm{Gr}_{g}} \rightarrow \mathbb{A}^{1}$ a $\mathbb{G}_{m}$-equivariant morphism, where $\mathbb{G}_{m}$ acts on $\mathbb{A}^{1}$ via natural dilatations (cf. [31, 24]). In addition, the restriction of this $\mathbb{G}_{m}$-action on $\left(\widetilde{\mathrm{Gr}}_{\mathscr{G}}\right)_{0}=\mathcal{F} \ell_{v}$ coincides with the action of the "rotation torus" on $\mathcal{F} \ell_{v}$ as mentioned in Lemma 1.2.

Then for any $\mathcal{F} \in \operatorname{Sat}_{H}$, the sheaf $\mathcal{G} \otimes \overline{\mathbb{Q}}_{\ell}[1]$ is $\mathbb{G}_{m}$-equivariant so that the monodromy of the nearby cycle $Z(\mathcal{F})=\Psi_{\widetilde{\mathrm{Gr}}_{\mathscr{g}}}\left(\mathcal{F} \otimes \overline{\mathbb{Q}}_{\ell}\right)$ is opposite to the $\mathbb{G}_{m}$-monodromic action on $\mathcal{Z}(\mathcal{F})$ 
(see [28] for the definition of $\mathbb{G}_{m}$-monodromic sheaves and $\mathbb{G}_{m}$-monodromic actions, and in particular Proposition 7.1 of loc. cit. for this statement). By Corollary 1.2, $Z(\mathcal{F})$ is $\mathbb{G}_{m}$-equivariant which exactly means that the monodromy is trivial.

Remark 2.4. - A mixed characteristic analogue of this lemma also holds (Theorem 7.2).

Let $i_{0}:(\widetilde{\operatorname{Gr}} g)_{0} \rightarrow \widetilde{\operatorname{Gr}} g$ be the closed embedding of the special fiber and $j: \widetilde{\operatorname{Gr}}_{g} \backslash\left(\widetilde{\operatorname{Gr}}_{g}\right)_{0} \rightarrow \widetilde{\operatorname{Gr}}_{g}$ be the open complement.

COROLlary 2.5. - There is a canonical isomorphism $\mathcal{Z}(\mathcal{F}) \simeq i_{0}^{*} j_{! *}\left(\mathcal{F} \otimes \overline{\mathbb{Q}}_{\ell}\right)$.

Proof. - This is standard. Since the monodromy is trivial, from the distinguished triangle

$$
i_{0}^{*} j_{*}\left(\mathcal{F} \otimes \overline{\mathbb{Q}}_{\ell}\right) \rightarrow Z \mathcal{Z}(\mathcal{F}) \stackrel{0}{\rightarrow} Z(\mathcal{F}) \rightarrow
$$

we obtain that $i_{0}^{*} j_{*}\left(\mathscr{F} \otimes \overline{\mathbb{Q}}_{\ell}\right)$ lives in perverse cohomological degree 0 and 1 , and both cohomology sheaves are isomorphic to $\mathcal{Z}(\mathcal{F})$. But $i_{0}^{*} j_{! *}\left(\mathcal{F} \otimes \overline{\mathbb{Q}}_{\ell}\right)={ }^{p} \mathrm{H}^{0} i_{0}^{*} j_{*}\left(\mathcal{F} \otimes \overline{\mathbb{Q}}_{\ell}\right)$, where ${ }^{p} \mathrm{H}^{*}$ stands for the perverse cohomology.

In what follows, for $\mathcal{F} \in \mathrm{Sat}_{H}$, we denote $\mathcal{F}_{\mathbb{G}_{m}}=\mathcal{F} \otimes \overline{\mathbb{Q}}_{\ell}[1]$ over $\left.\widetilde{\mathrm{Gr}_{g}}\right|_{\mathbb{G}_{m}}$, and $\mathcal{F}_{\mathbb{A}^{1}}=j_{! *} \mathcal{F}_{\mathbb{G}_{m}}$.

Recall that the irreducible objects in $\operatorname{Sat}_{H}$ are the intersection cohomology sheaves $\phi\left(V_{\mu}\right)$ on $\mathrm{Gr}_{H}$ where $\mu \in \mathbb{X}_{\bullet}\left(T_{H}\right)^{+}=\mathbb{X}_{\bullet}(T)^{+}$. On the other hand, the irreducible objects in $\mathscr{P}_{v}$ are the intersection cohomology sheaves $\operatorname{IC}_{\bar{\mu}}$ on $\mathcal{F} \ell_{v}$, where $\bar{\mu} \in \mathbb{X}_{\bullet}(T)_{I}^{+}$. For $\bar{\mu} \in \mathbb{X} \bullet(T)_{I}^{+}$, let $j_{\bar{\mu}}: \stackrel{\mathscr{F}}{\ell_{v \bar{\mu}}} \rightarrow \mathcal{F} \ell_{v}$ be the corresponding locally closed embedding of $K_{v}$-orbits.

Lemma 2.6. - For any $\mu \in \mathbb{X}_{\bullet}(T)^{+}$, let $\bar{\mu}$ be its image in $\mathbb{X}_{\bullet}(T)_{I}^{+}$. Then $j_{\bar{\mu}}^{*} Z\left(\varnothing\left(V_{\mu}\right)\right) \simeq \overline{\mathbb{Q}}_{\ell}[(2 \rho, \mu)]$.

Proof. - Consider $s_{\mu} \times \mathbb{G}_{m} \subset \mathrm{Gr}_{H} \times\left.\mathbb{G}_{m} \subset \widetilde{\mathrm{Gr}_{\mathscr{g}}}\right|_{\mathbb{G}_{m}}$. Since $\widetilde{\mathrm{Gr}_{\mathscr{g}}}$ is ind-proper over $\mathbb{A}^{1}$, it extends to a section $\mathbb{A}^{1} \rightarrow \widetilde{\mathrm{Gr}_{g}}$, still denoted by $s_{\mu}$. By [31, Proposition 3.5], $s_{\mu}(0) \in \mathcal{F} \ell_{v}$ is just the point $s_{\bar{\mu}}$, where $\bar{\mu}$ is the image of $\mu$ under $\mathbb{X}_{\bullet}\left(T_{H}\right) \rightarrow \mathbb{X}_{\bullet}\left(T_{H}\right)_{I}$.

Recall that $\delta\left(V_{\mu}\right)$ is supported on $\overline{\mathrm{Gr}}_{\mu}$, the Schubert variety in $\mathrm{Gr}_{H}$ corresponding to $s_{\mu}$. Let $\widetilde{\mathrm{Gr}}_{\mu} \subset \widetilde{\mathrm{Gr}}_{\mathscr{g}}$ be the closure of $\overline{\mathrm{Gr}}_{\mu} \times\left.\mathbb{G}_{m} \subset \widetilde{\mathrm{Gr}}_{\mathscr{g}}\right|_{\mathbb{G}_{m}}$. Then by the above fact, $\mathcal{F} \ell_{v \bar{\mu}}$ is contained in the special fiber of $\widetilde{\mathrm{Gr}}_{\mu}$. In fact, it is proved in [31] that the special fiber of $\widetilde{\mathrm{Gr}}_{\mu}$ is $\mathcal{F} \ell_{v \bar{\mu}}$. In addition, it is shown in loc. cit. that the point $s_{\bar{\mu}}$ is smooth in $\widetilde{\mathrm{Gr}}_{\mu}$. The lemma then is clear.

The following key result is established in [8] for the split case and in [31, Theorem 7.3] in general. Let $D_{K_{v}}\left(\mathcal{F} \ell_{v}\right)$ be the $K_{v}$-equivariant derived category on $\mathcal{F} \ell_{v}$, and $\star: \mathscr{P}_{v} \times \mathscr{P}_{v} \rightarrow D_{K_{v}}\left(\mathcal{F} \ell_{v}\right)$ be the convolution product functor. For a precise definition of the convolution product, see for example [19,31].

Proposition 2.7. - For any $\mathcal{F}_{1} \in \mathrm{Sat}_{H}$ and $\mathcal{F}_{2} \in \mathscr{P}_{v}$, there is a canonical isomorphism $Z\left(\mathcal{F}_{1}\right) \star \mathcal{F}_{2} \simeq \mathcal{F}_{2} \star \mathcal{Z}\left(\mathcal{F}_{1}\right)$ and both are objects in $\mathscr{P}_{v}$.

A brief review of the construction of this canonical isomorphism is given in $\S 3$.

Corollary 2.8. - The convolution of $\mathscr{P}_{v}$ is bi-exact. Therefore, $\mathscr{P}_{v}$ is a monoidal category. 
Proof. - Observe that $\mathscr{P}_{v}$ is semi-simple (Lemma 1.1) and every irreducible object in $\mathscr{P}_{v}$ is a direct summand of some $\mathcal{Z}(\mathcal{F})$. Indeed, for $\bar{\mu} \in \mathbb{X}_{\bullet}(T)_{I}^{+}$, let $\mu$ be a lift of it in $\mathbb{X}_{\bullet}(T)^{+}$. Then by Lemma $2.6, \mathrm{IC}_{\mu}$ appears as a direct summand of $\mathcal{Z}\left(\phi\left(V_{\mu}\right)\right)$ with multiplicity one. As the convolution (for left and right) with $\mathcal{Z}\left(\phi\left(V_{\mu}\right)\right)$ is exact, the convolution with its direct summand is also exact. The first claim follows.

It is well-known that the convolution functor $D_{K_{v}}\left(\mathcal{F} \ell_{v}\right) \times D_{K_{v}}\left(\mathcal{F} \ell_{v}\right) \rightarrow D_{K_{v}}\left(\mathcal{F} \ell_{v}\right)$ is monoidal. Its restriction to $\mathscr{P}_{v} \times \mathscr{P}_{v}$ takes value in $\mathscr{P}_{v}$. As $\mathscr{P}_{v}$ is a full subcategory of $D_{K_{v}}\left(\mathcal{F} \ell_{v}\right)$, the associativity constraints are morphisms in $\mathscr{P}_{v}$. Therefore, $\mathscr{P}_{v}$ is a monoidal subcategory of $D_{K_{v}}\left(\mathcal{F} \ell_{v}\right)$.

Remark 2.9. - (i) According to [19, Remark 4.5], the exactness of the convolution product probably would imply that $L G \times{ }^{K} \mathcal{F} \ell_{v} \rightarrow \mathcal{F} \ell_{v}$ is (stratified) semi-small.

(ii) By the same argument, the convolution bi-functor $\star: \mathscr{P}\left(\mathscr{F} \ell_{v}\right) \times \mathscr{P}_{v} \rightarrow \mathscr{P}\left(\mathcal{F} \ell_{v}\right)$ is also exact, where $\mathscr{P}\left(\mathcal{F} \ell_{v}\right)$ is the category of perverse sheaves on $\mathcal{F} \ell_{v}$.

\section{3. $Z$ is a central functor}

In this section, we show that $Z$ is a central functor in the sense of [2]. By Lemma 2.6, together with some general nonsense, this already implies that $\mathscr{P}_{v}$ is equivalent to $\operatorname{Rep}\left(\tilde{G}^{\vee}\right)$ for some closed subgroup $\tilde{G}^{\vee} \subset H^{\vee}$. In the next section, we will identify $\tilde{G}^{\vee}$ explicitly. We will also determine a fiber functor of $\mathscr{P}_{v}$.

Theorem-Definition 3.1. - The functor $\mathcal{Z}:$ Sat $_{H} \rightarrow \mathscr{P}_{v}$ is naturally a monoidal functor.

Proof. - The proof is literally the same as the proof in [8, Theorem 1(c)]. We repeat the argument here in order to make the definition of this monoidal structure explicit.

Let $\operatorname{Gr}_{\mathscr{g}} \tilde{\times} \mathrm{Gr}_{\mathscr{g}}$ be the ind-scheme over $\mathbb{A}^{2}$ classifying

$$
\operatorname{Gr}_{\mathscr{g}} \tilde{\times} \operatorname{Gr}_{\mathscr{g}}(R)=\left\{\begin{array}{l|l}
\left(x, y, \mathcal{E}, \mathcal{E}^{\prime}, \beta, \beta^{\prime}\right) & \begin{array}{c}
x, y \in \mathbb{A}^{1}(R), \mathcal{E}, \mathcal{E}^{\prime} \text { are two } \mathscr{G} \text {-torsors } \\
\text { on } \mathbb{A}_{R}^{1}, \beta:\left.\left.\mathcal{E}\right|_{\mathbb{A}_{R}^{1}-\Gamma_{x}} \simeq \mathcal{E}^{0}\right|_{\mathbb{A}_{R}^{1}-\Gamma_{x}} \text { is a } \\
\text { trivialization, } \beta^{\prime}:\left.\left.\mathcal{E}^{\prime}\right|_{\mathbb{A}_{R}^{1}-\Gamma_{y}} \simeq \mathcal{E}\right|_{\mathbb{A}_{R}^{1}-\Gamma_{y}}
\end{array}
\end{array}\right\} .
$$

Let $\operatorname{Gr}_{\mathscr{G}} \tilde{\mathbb{A}^{1}} \operatorname{Gr}_{\mathscr{G}}$ denote the restriction of $\operatorname{Gr}_{\mathscr{G}} \tilde{\times} \mathrm{Gr}_{\mathscr{G}}$ along the diagonal $\Delta \rightarrow \mathbb{A}^{2}$. Then $\operatorname{Gr}_{\mathscr{G}} \tilde{\mathbb{A}^{1}} \mathrm{Gr}_{\mathscr{G}}$ is a kind of twisted product. Indeed, let $\mathscr{L}^{+} \mathscr{G}$ be the global jet group of $\mathscr{G}$, which classifies a point on $\mathbb{A}^{1}$ and a trivialization of the trivial $\mathscr{G}$-torsor over the formal neighborhood of this point (cf. [31, §3.1]). Then $\mathcal{L}^{+} \mathscr{G}$ naturally acts on $\mathrm{Gr}_{\mathscr{g}}$. In addition, there is a $\mathcal{L}^{+} \mathscr{G}$-torsor over $\operatorname{Gr}_{\mathscr{g}}$ classifying quadruples $(y, \mathcal{E}, \beta, \gamma)$, where the triple $(y, \mathcal{E}, \beta)$ is as in the definition of $\mathrm{Gr}_{\mathscr{g}}$ and $\gamma$ is a trivialization of $\mathcal{E}$ over the formal neighborhood of $y$ (this is indeed the global loop group $\mathscr{L} \mathscr{G}$ of $\mathscr{G}$ introduced in [31, §3.1]). Then $\operatorname{Gr}_{\mathscr{G}} \tilde{\mathbb{A}^{1}} \operatorname{Gr}_{\mathscr{G}} \simeq \mathscr{L} \mathscr{G}^{\mathcal{L}^{+} \mathscr{\mathscr { G }}} \mathrm{Gr}_{\mathscr{G}}$. Let us denote the base change of this isomorphism along

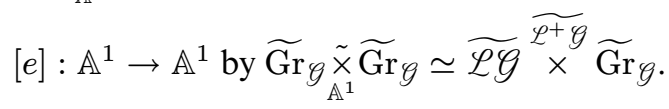


Let $\mathcal{F}_{1}$ and $\mathcal{F}_{2}$ be two objects in $\operatorname{Sat}_{H}$. We can form the twisted product $\left(\mathcal{F}_{1}\right)_{\mathbb{G}_{m}} \tilde{\times}\left(\mathcal{F}_{2}\right)_{\mathbb{G}_{m}}$

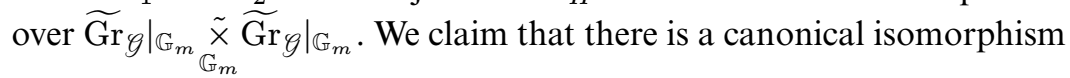

$$
\Psi_{\widetilde{\operatorname{Gr}}_{\mathscr{G}} \tilde{\mathbb{A}^{1}} \widetilde{\operatorname{Gr}_{\mathscr{g}}}}\left(\left(\mathcal{F}_{1}\right)_{\mathbb{G}_{m}} \tilde{\times}\left(\mathcal{F}_{2}\right)_{\mathbb{G}_{m}}\right) \simeq \mathcal{Z}\left(\mathcal{F}_{1}\right) \tilde{\times} \mathcal{Z}\left(\mathcal{F}_{2}\right) .
$$

Indeed, let $V_{i} \subset \widetilde{\mathrm{Gr}}_{\mathscr{g}}$ be the closure of the support of $\mathcal{F}_{i} \otimes \overline{\mathbb{Q}}_{\ell}[1]$ in $\left.\widetilde{\mathrm{Gr}}_{\mathscr{g}}\right|_{\mathbb{G}_{m}}$. Let $\mathscr{L}_{n}^{+} \mathscr{G}$ be the $n$th jet group such that the action of $\mathcal{L}^{+} \mathscr{G}$ on $V_{i}$ factors through $\mathscr{L}_{n}^{+} \mathscr{G}$. The corresponding $\mathscr{L}_{n}^{+} \mathscr{G}$ torsor over $\operatorname{Gr}_{\mathscr{G}}$ is denoted by $\mathscr{L}_{n} \mathscr{G}$. Let us denote by $\widetilde{\mathscr{L}_{n}^{+} \mathscr{G}}$ and $\widetilde{\mathcal{L}_{n} \mathscr{G}}$ their base changes along $[e]$. Then one can check the isomorphism after pullback along

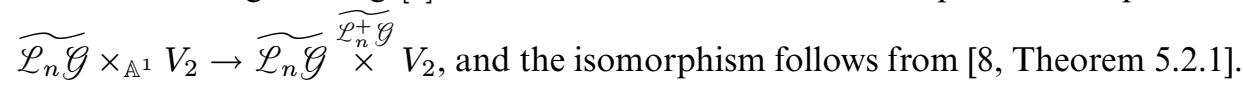

Now $\operatorname{Gr}_{\mathscr{G}} \tilde{\mathbb{A}^{1}} \operatorname{Gr}_{\mathscr{G}} \rightarrow \operatorname{Gr}_{\mathscr{g}},\left(y, \mathcal{E}, \mathcal{E}^{\prime}, \beta, \beta\right) \mapsto\left(y, \mathcal{E}^{\prime}, \beta \beta^{\prime}\right)$ is ind-proper and taking nearby cycles commutes with proper push-forward. Therefore we obtain the canonical isomorphism

$$
\mathcal{Z}\left(\mathcal{F}_{1} \star \mathcal{F}_{2}\right) \simeq Z\left(\mathcal{F}_{1}\right) \star Z Z Z\left(\mathcal{F}_{2}\right) \text {. }
$$

In addition, working over $\operatorname{Gr}_{\mathscr{g}} \underset{\mathbb{A}^{1}}{\tilde{\times}} \operatorname{Gr}_{\mathscr{g}} \underset{\mathbb{A}^{1}}{\tilde{\times}} \mathrm{Gr}_{\mathscr{g}}$, one can see that this isomorphism makes $Z$ a monoidal functor.

Let us recall the definition of central functors as in [2]. Namely, if $F: \mathscr{C} \rightarrow \mathscr{D}$ is a monoidal functor between two monoidal categories and assume that $\mathscr{C}$ is a symmetric monoidal category, then $F$ (together with the following data) is called central if

1. there is an isomorphism $c$ of the bi-functors $\mathscr{C} \times \mathscr{D} \rightarrow \mathscr{D},(X, Y) \mapsto F(X) \otimes Y$ and $(X, Y) \mapsto Y \otimes F(X)$, i.e., an isomorphism $c_{X, Y}: F(X) \otimes Y \simeq Y \otimes F(X)$ functorial in $X, Y$;

2. for $X, X^{\prime} \in \mathscr{C}$, the following diagram is commutative

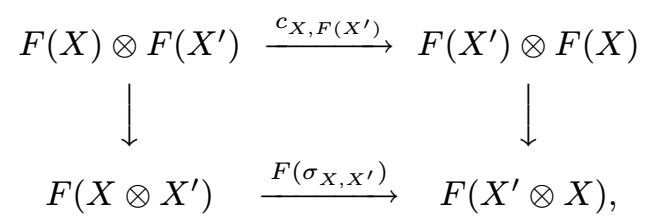

where $\sigma$ is the commutativity constraint of $\mathscr{C}$;

3. for $X \in \mathscr{C}$ and $Y, Y^{\prime} \in \mathscr{D}$, the following diagram is commutative

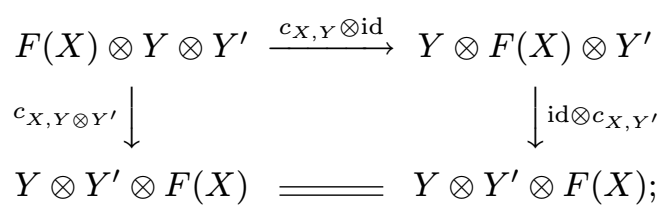

4. for $X, X^{\prime} \in \mathscr{C}, Y \in \mathscr{D}$, the following diagram is commutative

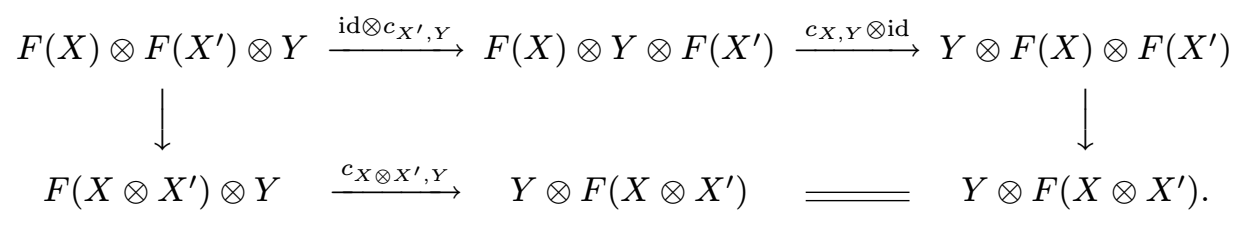

$4^{\mathrm{e}}$ SÉRIE - TOME $48-2015-\mathrm{N}^{\circ} 2$ 
Proposition 3.2. - The functor Z, together with the canonical isomorphisms provided in Proposition 2.7, is a central functor. There is an algebraic group $\tilde{G}^{\vee} \subset H^{\vee}$ together with an equivalence $S: \mathscr{P}_{v} \simeq \operatorname{Rep}\left(\tilde{G}^{\vee}\right)$ such that $S \circ Z \simeq \operatorname{Res}_{H^{\vee}}^{\tilde{G}^{\vee}}$ as tensor functors, where $\operatorname{Res}_{H^{\vee}}^{\tilde{G}^{\vee}}$ is the restriction functor from $\operatorname{Rep}\left(H^{\vee}\right)$ to $\operatorname{Rep}\left(\tilde{G}^{\vee}\right)$.

Proof. - Since every object in $\mathscr{P}_{v}$ appears as a direct summand of some object in the essential image of $\mathcal{Z}$, the second statement of the proposition is a direct consequence of the first statement and Proposition 1 of [2].

The first statement can be checked literally the same as in [9]. For this, let us briefly review the construction of the canonical isomorphisms in Proposition 2.7. Let us define the Beilinson-Drinfeld Grassmannian

$$
\operatorname{Gr}_{\mathscr{G}}^{B D}(R)=\left\{\begin{array}{l|l}
(y, \mathcal{E}, \beta) & \begin{array}{l}
y \in \mathbb{A}^{1}(R), \mathcal{E} \text { is a } \mathscr{G} \text {-torsor on } \mathbb{A}_{R}^{1}, \text { and } \\
\beta:\left.\left.\mathcal{E}\right|_{\left(\mathbb{G}_{m}\right)_{R}-\Gamma_{y}} \simeq \mathcal{E}^{0}\right|_{\left(\mathbb{G}_{m}\right)_{R}-\Gamma_{y}} \text { is a trivialization }
\end{array}
\end{array}\right\},
$$

where $\mathcal{E}^{0}$ denotes the trivial torsor. This is the restriction to $\{0\} \times \mathbb{A}^{1}$ (or to $\mathbb{A}^{1} \times\{0\}$ ) of the usual Beilinson-Drinfeld Grassmannian over $\mathbb{A}^{2}$ (e.g., the one considered in [19]). In particular, we have

$$
\left.\left.\operatorname{Gr}_{\mathscr{G}}^{B D}\right|_{\mathbb{G}_{m}} \simeq \operatorname{Gr}_{\mathscr{G}}\right|_{\mathbb{G}_{m}} \times\left(\operatorname{Gr}_{\mathscr{G}}\right)_{0}, \quad\left(\operatorname{Gr}_{\mathscr{G}}^{B D}\right)_{0} \simeq\left(\operatorname{Gr}_{\mathscr{G}}\right)_{0} \simeq \mathcal{F} \ell_{v}
$$

As before, we denote by $\widetilde{\mathrm{Gr}}_{\mathscr{g}}^{B D}$ the base change along $[e]: \mathbb{A}^{1} \rightarrow \mathbb{A}^{1}$. Over $\left.\widetilde{\mathrm{Gr}}_{\mathscr{g}}^{B D}\right|_{\mathbb{G}_{m}} \simeq$ $\left.\widetilde{\operatorname{Gr}}_{\mathscr{g}}\right|_{\mathbb{G}_{m}} \times\left(\operatorname{Gr}_{\mathscr{g}}\right)_{0}$, we can form the external product $\left(\mathcal{F}_{1}\right)_{\mathbb{G}_{m}} \otimes \mathcal{F}_{2}$. Then the isomorphism in the proposition is induced from the canonical isomorphisms

$$
\mathcal{Z}\left(\mathcal{F}_{1}\right) \star \mathcal{F}_{2} \simeq \Psi_{\widetilde{\mathrm{Gr}}_{\mathscr{g}}^{B D}}\left(\left(\mathcal{F}_{1}\right)_{\mathbb{G}_{m}} \otimes \mathcal{F}_{2}\right) \simeq \mathcal{F}_{2} \star \mathcal{Z}\left(\mathcal{F}_{1}\right)
$$

where the two isomorphisms are induced from the natural maps

$$
\left.\left.\operatorname{Gr}_{\mathscr{g}} \tilde{\times} \operatorname{Gr}_{\mathscr{g}}\right|_{\mathbb{A}^{1} \times\{0\}} \rightarrow \operatorname{Gr}_{\mathscr{g}}^{B D} \leftarrow \operatorname{Gr}_{\mathscr{g}} \tilde{\times} \operatorname{Gr}_{\mathscr{g}}\right|_{\{0\} \times \mathbb{A}^{1}} .
$$

See [31, Proposition 7.4] for more details.

Again, since the monodromy of $\Psi_{\widetilde{G r}_{\mathscr{G}}^{B D}}\left(\left(\mathcal{F}_{1}\right)_{\mathbb{G}_{m}} \otimes \mathcal{F}_{2}\right)$ is trivial, we have

$$
\mathcal{Z}\left(\mathcal{F}_{1}\right) \star \mathcal{F}_{2} \simeq i_{0}^{*} j_{! *}\left(\left(\mathcal{F}_{1}\right)_{\mathbb{G}_{m}} \otimes \mathcal{F}_{2}\right) \simeq \mathcal{F}_{2} \star \mathcal{Z}\left(\mathcal{F}_{1}\right),
$$

where $i_{0}, j$ are corresponding closed and open embedding.

Back to the proof of the proposition. The conditions (3) and (4) are checked the same way as in [9]. To check condition (2) is even simpler than loc. cit. Observe that the monodromy of all the nearby cycles involved is trivial. Then the nearby cycles can be expressed via intermediate extensions as in Corollary 2.5 and (3.3), rather than via the homotopy (co)limits of certain ind-pro system of sheaves as in loc. cit.

Now we would like to endow $\mathscr{P}_{v}$ with a fiber functor. We begin with the following general lemma.

Lemma 3.3. - Let $G_{1} \subset G_{2}$ be a closed embedding of affine algebraic groups over a field $E$ (of characteristic zero). Let $F: \operatorname{Rep}\left(G_{1}\right) \rightarrow \operatorname{Vect}_{E}$ be an E-linear exact and faithful functor. Assume that: (i) $F(X \otimes Y)$ and $F(X) \otimes F(Y)$ are (non-canonically) isomorphic; (ii) $F \circ \operatorname{Res}_{G_{2}}^{G_{1}}$ is a fiber functor of $\operatorname{Rep}\left(G_{2}\right)$. Then $F$ has a unique fiber functor structure which induces the fiber functor structure of $F \circ \operatorname{Res}_{G_{2}}^{G_{1}}$ as in (ii). 
REMARK 3.4. - We are not sure whether the first assumption is necessary.

Proof. - The uniqueness is clear. We write $R=\operatorname{Res}_{G_{2}}^{G_{1}}$ for simplicity. For any $X \in \operatorname{Rep}\left(G_{2}\right)$, let $\langle X\rangle$ denote the full subcategory of $\operatorname{Rep}\left(G_{2}\right)$ consisting of the objects that are isomorphic to subquotients of $X^{n}, n \in \mathbb{N}$, and $\langle R(X)\rangle$ denote the full subcategory of $\operatorname{Rep}\left(G_{1}\right)$ consisting of the objects that are isomorphic to subquotients of $R(X)^{n}, n \in \mathbb{N}$. Let us denote $\operatorname{End}\left(\left.F R\right|_{\langle X\rangle}\right)\left(\right.$ resp. $\left.\operatorname{End}\left(\left.F\right|_{\langle R(X)\rangle}\right)\right)$ the endomorphism algebra of the restriction of the functor $F R$ (resp. $F$ ) to $\langle X\rangle$ (resp. $\langle R(X)\rangle$ ). They are finite dimensional $E$-algebras and clearly, the $E$-algebra homomorphism $\operatorname{End}\left(\left.F\right|_{\langle R(X)\rangle}\right) \rightarrow \operatorname{End}\left(\left.F R\right|_{\langle X\rangle}\right)$ is injective. According to [7, Lemma 2.13], there are canonical equivalences and the following commutative diagram

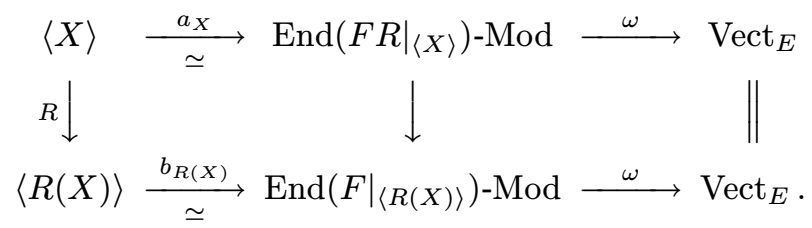

In addition, $\omega a_{X} \simeq F R$ and $\omega b_{R(X)} \simeq F$. Observe that if $\langle X\rangle$ is a subcategory of $\langle Y\rangle$. Then we have a natural algebra homomorphism $\operatorname{End}\left(\left.F R\right|_{\langle Y\rangle}\right) \rightarrow \operatorname{End}\left(\left.F R\right|_{\langle X\rangle}\right)$. Then $A=\underline{\lim }_{X \in \operatorname{Rep} G_{2}} \operatorname{End}\left(\left.F R\right|_{\langle X\rangle}\right)^{\vee}$ is a coalgebra. Similarly, we can define $B=\varliminf_{X \in \operatorname{Rep} G_{2}} \operatorname{End}\left(\left.F\right|_{\langle R(X)\rangle}\right)^{\vee}$. We have the surjective map of coalgebras $A \rightarrow B$, and

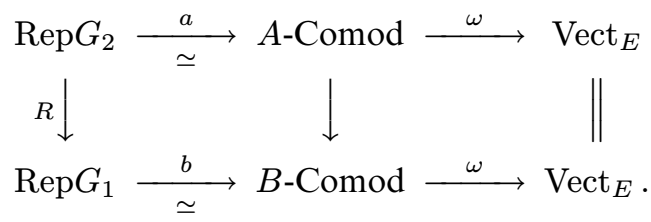

By the assumption (i) and [7, Proposition 2.16], the tensor structures on $\operatorname{Rep} G_{1}$ and $\operatorname{Rep} G_{2}$ induce $B \otimes B \rightarrow B$ and $A \otimes A \rightarrow A$ respectively. Since the restriction functor $R$ is a tensor functor, we have the commutative diagram

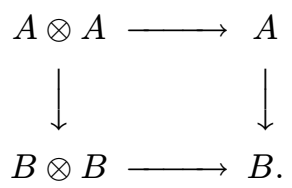

By assumption (ii), $\omega a \simeq F R$ is a fiber functor of $\operatorname{Rep} G_{2}$, and therefore we know that $A=\theta_{G_{2}}$ and the map $A \otimes A \rightarrow A$ is the usual multiplication. Since the map $A \rightarrow B$ is surjective, this implies that the map $B \otimes B \rightarrow B$ is also associative and commutative. By [7, Proposition 2.16] again, this implies that the functor $F$ is compatible with the associativity and the commutativity constraints. The lemma follows.

COROLlary 3.5. - The functor given by taking the cohomology $\mathrm{H}^{*}: \mathscr{P}_{v} \rightarrow \operatorname{Vect}_{\overline{\mathbb{Q}}_{\ell}}$ has a natural structure as a fiber functor.

Proof. - It is well-known (e.g., from the decomposition theorem) that there exists an isomorphism $\mathrm{H}^{*}\left(\mathcal{F} \ell_{v}, \mathcal{F}_{1} \star \mathcal{F}_{2}\right) \simeq \mathrm{H}^{*}\left(\mathcal{F}_{\ell_{v}}, \mathcal{F}_{1}\right) \otimes \mathrm{H}^{*}\left(\mathcal{F} \ell_{v}, \mathcal{F}_{2}\right)$ (non-canonically). Since taking nearby cycles commutes with proper push-forward, we have a canonical isomorphism 
$\mathrm{H}^{*} \circ Z \simeq \mathrm{H}^{*}$. Since $\mathrm{H}^{*}: \operatorname{Sat}_{H} \rightarrow \operatorname{Vect}_{\overline{\mathbb{Q}}_{\ell}}$ is a fiber functor, the assertion follows from the above lemma.

\section{Identification of the group $\tilde{G}^{\vee}$ with $\left(H^{\vee}\right)^{I}$}

We need to describe the group $\tilde{G}^{\vee}$ from the last section. To begin with, let us review how the geometric Satake correspondence (together with a choice of an ample line bundle on $\left.\mathrm{Gr}_{H}\right)$ gives rise to a pinned group $\left(H^{\vee}, B_{H}^{\vee}, T_{H}^{\vee}, X^{\vee}\right)$. First, once we choose $T_{H} \subset B_{H} \subset H$, the construction of [19] gives us $T_{H}^{\vee} \subset B_{H}^{\vee} \subset H^{\vee}$. Namely, let $U_{H} \subset B_{H}$ be its unipotent radical. For $\mu \in \mathbb{X}_{\bullet}\left(T_{H}\right)$, let $S_{\mu}$ be the semi-infinite orbit on $\operatorname{Gr}_{H}$ passing through $s_{\mu}$ as introduced in [19] (i.e., the $L U_{H}$-orbit passing through $s_{\mu}$ ). Let $S_{\leq \mu}=\cup_{\lambda \preceq \mu} S_{\lambda}$ and $S_{<\mu}=\cup_{\lambda \prec \mu} S_{\lambda}$. Then the fiber functor $\mathrm{H}^{*}: \mathrm{Sat}_{H} \rightarrow$ Vect $_{\mathbb{Q}_{\ell}}$ has a canonical filtration (called the $M V$ filtration) given by $\operatorname{ker}\left(\mathrm{H}^{*}\left(\mathrm{Gr}_{H},-\right) \rightarrow \mathrm{H}^{*}\left(S_{<\mu},-\right)\right)$. This defines a Borel $B_{H}^{\vee} \subset H^{\vee}$. In addition, it is proved that the filtration admits a canonical splitting, i.e., a canonical isomorphism $\mathrm{H}^{*}\left(\mathrm{Gr}_{H},-\right) \simeq \bigoplus_{\mu} \mathrm{H}_{c}^{*}\left(S_{\mu},-\right)$. This provides a maximal torus $T_{H}^{\vee} \subset B_{H}^{\vee}$. Let $\mathscr{L}$ be an ample line bundle on $\mathrm{Gr}_{H}$, and let $c(\mathscr{L}) \in \mathrm{H}^{2}\left(\mathrm{Gr}_{H}, \overline{\mathbb{Q}}_{\ell}\right)$ be its Chern class. Then it is shown in [10,29] that the cup product with this class realizes $c(\mathscr{L})$ as a principal nilpotent element in $X^{\vee} \in \mathfrak{h}^{\vee}=\operatorname{Lie} H^{\vee}$. In addition, by [29, Proposition 5.6], the quadruple $\left(H^{\vee}, B_{H}^{\vee}, T_{H}^{\vee}, X^{\vee}\right)$ is indeed a pinned reductive group.

REMARK 4.1. - One remark is in order. In [29], all the assertions are proved for the affine Grassmannian defined over $\mathbb{C}$. The only place where the complex topology is used, besides the issue of dealing with $\mathbb{Z}$-coefficients as in [19], is to define the coproduct on $\mathrm{H}^{*}\left(\mathrm{Gr}_{H}, \mathbb{Z}\right)$ by realizing $\mathrm{Gr}_{H}$ as being homotopic to the based loop space of a maximal compact subgroup of $H_{\mathbb{C}}$. However, one can provide a commutative and cocommutative Hopf algebra structure on $\mathrm{H}^{*}\left(\mathrm{Gr}_{H}, \mathbb{Z}\right)$ using the Beilinson-Drinfeld Grassmannian. More precisely, one can use the isomorphism (2.11) in loc. cit. to define the comultiplication map by the formula (2.12) in loc. cit. This map on the other hand can be realized as follows. There is the Beilinson-Drinfeld Grassmannian $\pi: \mathrm{Gr}_{2} \rightarrow \mathbb{A}^{2}$ whose fiber over a point in the diagonal $\Delta \subset \mathbb{A}^{2}$ is $\mathrm{Gr}_{H}$ and whose fiber over a point off the diagonal is $\mathrm{Gr}_{H} \times \mathrm{Gr}_{H}$ (cf. [19, Section 5]). Then $R^{i} \pi_{*} \mathbb{Q}_{\ell}$ is a constructible sheaf on $\mathbb{A}^{2}$, constant along the stratification $\mathbb{A}^{2}=\Delta \cup\left(\mathbb{A}^{2}-\Delta\right)$. Now the usual cospecialization map of constructible sheaves gives rise to the comultiplication. From this latter definition, the usual arguments for the commutativity constraints as in [19] show that this defined comultiplication is indeed cocommutative. The proof of [29, Lemma 5.1] that $c^{T_{H}}(\mathscr{L})$ is primitive under this Hopf algebra structure can be replaced by the following argument: as is well-known (e.g., see [30, 1.1.9]), if $\mathscr{L}$ is ample on $\mathrm{Gr}_{H}$, then there is an ample line bundle on $\mathrm{Gr}_{2}$, which away from the diagonal is $\mathscr{L} \otimes \mathscr{L}$ and on the diagonal is $\mathscr{L}$. Now the above arguments and all the remaining arguments of [29] apply to the situation when $\mathrm{Gr}_{H}$ is defined over arbitrary field $k$ and sheaves have $\overline{\mathbb{Q}}_{\ell}$-coefficients.

REMARK 4.2. - As explained in [19, Theorem 3.6], the above pinning $\left(H^{\vee}, B_{H}^{\vee}, T_{H}^{\vee}, X^{\vee}\right)$ is in fact independent of the choice of $T \subset B$. Another way to deduce this fact is as follows. The natural grading on the cohomological functor $\mathrm{H}^{*}$ defines a one-parameter subgroup $\mathbb{G}_{m} \rightarrow H^{\vee}$ and $T_{H}^{\vee}$ is just the centralizer of this subgroup, which is independent of the choice of $T \subset B$. On the other hand, $B_{H}^{\vee}$ is completely determined by $X^{\vee}$, which is also 
independent of the choice of $T \subset B$. In other words, there is a canonical morphism from the Lefschetz $\mathrm{SL}_{2}$ to $H^{\vee}$, which gives a principal $\mathrm{SL}_{2}$ in $H^{\vee}$, and the pinning is determined by this principal $\mathrm{SL}_{2}$.

Recall that we denote by $\psi$ the action of $I$ on $\mathrm{Gr}_{H}$. The action of $\gamma \in I$ will map $\overline{\mathrm{Gr}}_{\lambda}$ isomorphically to $\overline{\mathrm{Gr}}_{\gamma(\lambda)}$. Therefore the pushforward functor $\gamma_{*}: D\left(\mathrm{Gr}_{H}\right) \rightarrow D\left(\mathrm{Gr}_{H}\right)$ for $\gamma \in I$ naturally gives rise to $\gamma_{*}: \operatorname{Sat}_{H} \rightarrow \operatorname{Sat}_{H}$. In this way, $I$ acts on $\operatorname{Sat}_{H}$ via tensor automorphisms. Under the geometric Satake correspondence, $I$ acts on $H^{\vee}$ clearly as pinned automorphisms with respect to the pinning we mentioned above.

THEOREM 4.3. $-\tilde{G}^{\vee} \simeq\left(H^{\vee}\right)^{I}$.

REMARK 4.4. - Observe that $\left(H^{\vee}\right)^{I}$ is not necessarily a connected reductive group. For example: let $H^{\vee}=\mathrm{GL}_{2 n+1}$, let $J$ be the matrix with $1 s$ on the anti-diagonal and $0 s$ elsewhere. Let $\Gamma=\{1, \gamma\}$ and $\gamma$ act on $H^{\vee}$ via $g \mapsto J\left(g^{t}\right)^{-1} J$. Then $\left(H^{\vee}\right)^{I}=\mathrm{O}_{2 n+1}$.

Proof. - Since $\mathscr{P}_{v}$ is semi-simple, $\tilde{G}^{\vee}$ is a reductive subgroup of $H^{\vee}$. We first see that $\tilde{G}^{\vee} \subset\left(H^{\vee}\right)^{I}$. The following lemma is a direct consequence of [7, Corollary 2.9].

Lemma 4.5. - Let $f: H_{2} \rightarrow H_{1}$ be a homomorphism of algebraic groups and let $\omega^{f}$ denote the induced tensor functor $\operatorname{Rep}\left(H_{1}\right) \rightarrow \operatorname{Rep}\left(H_{2}\right)$ (if $f$ is a closed embedding then $\omega^{f}$ is the restriction functor $\left.\operatorname{Res}_{H_{1}}^{H_{2}}\right)$. Let $I \subset \operatorname{Aut}\left(H_{1}\right)$ so that it acts on $\operatorname{Rep}\left(H_{1}\right)$ via tensor automorphisms. If for any $\gamma \in I, \omega^{f} \circ \omega^{\gamma} \simeq \omega^{f}$, then $f$ factors through $f: H_{2} \rightarrow H_{1}^{I} \subset H_{1}$.

Now, $I$ acts on $\widetilde{\operatorname{Gr}_{\mathscr{C}}}=\operatorname{Gr}_{\mathscr{g}} \times_{\mathbb{A}^{1}} \mathbb{A}^{1}$ via the action on the second factor $\mathbb{A}^{1}$ by deck transformations. By Lemma 2.1, we have

$Z\left(\gamma_{*} \mathcal{F}\right)=\Psi_{\widetilde{\mathrm{Gr}}_{\mathscr{g}}}\left(\gamma_{*} \mathcal{F} \otimes \overline{\mathbb{Q}}_{\ell}[1]\right) \simeq \Psi_{\widetilde{\mathrm{Gr}}_{\mathscr{f}}}\left((\psi(\gamma) \times \gamma)_{*}\left(\mathcal{F} \otimes \overline{\mathbb{Q}}_{\ell}[1]\right)\right) \simeq \Psi_{\widetilde{\mathrm{Gr}}_{\mathscr{g}}}\left(\mathcal{F} \otimes \overline{\mathbb{Q}}_{\ell}[1]\right)=\mathcal{Z}(\mathcal{F})$.

In other words, we have the tensor isomorphism between $Z \circ \gamma_{*}$ and $Z$ for all $\gamma \in I$. From the above lemma, $\tilde{G}^{\vee} \subset\left(H^{\vee}\right)^{I}$.

Therefore, we have successive restriction functors

$$
\operatorname{Rep}\left(H^{\vee}\right) \rightarrow \operatorname{Rep}\left(\left(H^{\vee}\right)^{I}\right) \rightarrow \operatorname{Rep}\left(\tilde{G}^{\vee}\right) .
$$

To prove that $\tilde{G}^{\vee}=\left(H^{\vee}\right)^{I}$, it is enough to show that the above restriction induces an isomorphism of $K$-groups $K\left(\operatorname{Rep}\left(H^{\vee}\right)^{I}\right) \simeq K\left(\operatorname{Rep}\left(\tilde{G}^{\vee}\right)\right)$.

As the group $\left(H^{\vee}\right)^{I}$ may not be connected, we need to be careful to describe its representation ring.

Let $\left(H^{\vee}\right)^{I, 0}$ denote the neutral connected component of $\left(H^{\vee}\right)^{I}$. This is a connected reductive group with maximal torus $\left(T^{\vee}\right)^{I, 0}$, the neutral connected component of $\left(T^{\vee}\right)^{I}$. The key fact is the following lemma.

Lemma 4.6. - The natural map

$$
\left(T^{\vee}\right)^{I} /\left(T^{\vee}\right)^{I, 0} \rightarrow\left(H^{\vee}\right)^{I} /\left(H^{\vee}\right)^{I, 0}
$$

is an isomorphism.

$4^{\text {e }}$ SÉRIE - TOME $48-2015$ - No 2 
Proof. - We will not distinguish a group from its $E$-points. As we purely work with dual groups, we switch the notation $H^{\vee}$ to $H$, etc. in the proof. Let us choose $\gamma$ to be a generator of $I$. We need to show that $\pi_{0}\left(T^{I}\right)=\pi_{0}\left(H^{I}\right)$. Let $N$ be the normalizer of $T$ in $H$ and let $W=N / T$ be the Weyl group. Then $I$ acts on $W$ naturally. Let us define a right action of $W^{I}$ on $\mathrm{H}^{1}(I, T)$ as follows. Suppose $w$ is in $W^{I}$ and $c$ be a cohomology class; lift $w$ to $n \in N$ and lift $c$ to a cocycle $\varphi: I \rightarrow T$. Then we set $(c \cdot w)(\gamma)=\left[n^{-1} \varphi(\gamma) \gamma(\mathrm{n})\right]$. It is clear that this is independent of all choices. We will deduce Lemma 4.6 from the following fact.

Lemma 4.7. - Under the above definition, every element $w \in W^{I}$ acts on $\mathrm{H}^{1}(I, T)$ via a group automorphism. In addition, $\mathrm{H}^{1}(I, H)$ is the quotient of $\mathrm{H}^{1}(I, T)$ via the above action.

Proof. - Observe that the map $N^{I} \rightarrow W^{I}$ is surjective. Indeed, let $H_{\text {der }}$ be the derived group of $H$ and $H_{\mathrm{sc}}$ be the simply-connected cover of $H_{\mathrm{der}}$. We have corresponding groups $N_{\mathrm{der}}, N_{\mathrm{sc}}, T_{\mathrm{der}}, T_{\mathrm{sc}}$. We now apply the argument of [26] p. 55 (5) to $H_{\mathrm{sc}}$ and $\gamma$. Our assumption that $\gamma$ is pinned allows us to take $t=1$ in loc. cit. It follows that the natural map $N_{\mathrm{sc}}^{I} \rightarrow W^{I}$ is surjective; therefore the same is true for $N^{I} \rightarrow W^{I}$ (another argument of this surjectivity can be found in [3, Lemma 6.2]). By taking the lift $w$ to $n \in N^{I}$, it is clear that $w$ acts on $\mathrm{H}^{1}(I, T)$ via group automorphisms. The second statement was proved in [24].

Corollary 4.8. - The preimage of $1 \in \mathrm{H}^{1}(I, H)$ under $\mathrm{H}^{1}(I, T) \rightarrow \mathrm{H}^{1}(I, H)$ is 1 .

We continue to prove Lemma 4.6. First, if $H$ is simply-connected, then $H^{I}$ is connected as is shown in [26, Theorem 8.2]. On the other hand, $I$ acts on $T$ via permuting a basis of $\mathbb{X} \bullet(T)$. Therefore, $T^{I}$ is also connected. The lemma holds in this case. For general $H$, let $H_{\text {der }}$ be the derived group of $H$ and $H_{\mathrm{sc}}$ be the simply-connected cover of $H_{\mathrm{der}}$. Let $T_{\mathrm{der}}$ and $T_{\mathrm{sc}}$ be the corresponding preimages of $T$. Write

$$
1 \rightarrow Z \rightarrow H_{\mathrm{sc}} \rightarrow H_{\mathrm{der}} \rightarrow 1
$$

which then gives

$$
1 \rightarrow \pi_{0}\left(H_{\mathrm{der}}^{I}\right) \rightarrow \mathrm{H}^{1}(I, Z) \rightarrow \mathrm{H}^{1}\left(I, H_{\mathrm{sc}}\right) .
$$

Similarly, the sequence of maximal tori

$$
1 \rightarrow Z \rightarrow T_{\mathrm{sc}} \rightarrow T_{\mathrm{der}} \rightarrow 1
$$

gives

$$
1 \rightarrow \pi_{0}\left(T_{\mathrm{der}}^{I}\right) \rightarrow \mathrm{H}^{1}(I, Z) \rightarrow \mathrm{H}^{1}\left(I, T_{\mathrm{sc}}\right) .
$$

Comparing (4.1) and (4.2) and applying Corollary 4.8, we obtain that the natural map

$$
\pi_{0}\left(T_{\mathrm{der}}^{I}\right) \stackrel{\sim}{\longrightarrow} \pi_{0}\left(H_{\mathrm{der}}^{I}\right)
$$

is an isomorphism. Now consider

$$
1 \rightarrow H_{\mathrm{der}} \rightarrow H \rightarrow D \rightarrow 1
$$

which gives

$$
1 \rightarrow \pi_{0}\left(H_{\mathrm{der}}^{I}\right) \rightarrow \pi_{0}\left(H^{I}\right) \rightarrow \pi_{0}\left(D^{I}\right) \rightarrow \mathrm{H}^{1}\left(I, H_{\mathrm{der}}\right)
$$


Similarly, the sequence of maximal tori

$$
1 \rightarrow T_{\mathrm{der}} \rightarrow T \rightarrow D \rightarrow 1
$$

give

$$
1 \rightarrow \pi_{0}\left(T_{\mathrm{der}}^{I}\right) \rightarrow \pi_{0}\left(T^{I}\right) \rightarrow \pi_{0}\left(D^{I}\right) \rightarrow \mathrm{H}^{1}\left(I, T_{\mathrm{der}}\right) .
$$

Comparing (4.4) and (4.5) and using Corollary 4.8 again, we obtain that the natural map

$$
\pi_{0}\left(T^{I}\right) \stackrel{\sim}{\longrightarrow} \pi_{0}\left(H^{I}\right)
$$

is an isomorphism.

Remark 4.9. - Note that the above proof of Lemma 4.6 in fact applies to any cyclic subgroup $I$ of the group of pinned automorphisms of $H$. With a little more effort, one can prove the same statement for any solvable subgroup. Also note that Lemma 4.7 implies that if $I$ acts on $H$ via pinned automorphisms, then $\mathrm{H}^{1}(I, H)$ has a canonical Abelian group structure. This does not necessarily hold for arbitrary action of $I$ on $H$.

Recall that there is a natural partial order “ $\preceq$ ” on $\mathbb{X} \bullet(T)_{I}$ given by $(1.3)$. Let $\bar{\mu} \in \mathbb{X} \bullet(T)_{I}^{+}$. We say an irreducible representation $W$ of $\left(H^{\vee}\right)^{I}$ is of highest weight $\bar{\mu}$ if $\bar{\mu}$ appears as a weight under the weight decomposition of $W$ with respect to $\left(T^{\vee}\right)^{I}$ and any other weight appearing in this weight decomposition is $\preceq \bar{\mu}$. We claim that

Lemma 4.10. - (i) For $\bar{\mu} \in \mathbb{X}_{\bullet}(T)_{I}^{+}$, there is a unique (up to isomorphism) irreducible representation $W_{\bar{\mu}}$ of $\left(H^{\vee}\right)^{I}$ of highest weight $\bar{\mu}$. In addition, any irreducible representation of $\left(H^{\vee}\right)^{I}$ is of this form.

(ii) The multiplicity of the $\bar{\mu}$-weight in $W_{\bar{\mu}}$ is one.

Proof. - Indeed, let $\overline{\bar{\mu}}$ be the image of $\bar{\mu}$ in $\mathbb{X}_{\bullet}(T)_{I} / \mathbb{X}_{\bullet}(T)_{I, \text { tor }}$, and $W_{\overline{\bar{\mu}}}$ be the unique irreducible representation of $\left(H^{\vee}\right)^{I, 0}$ of highest weight $\overline{\bar{\mu}}$. Let $W$ be an irreducible represen-

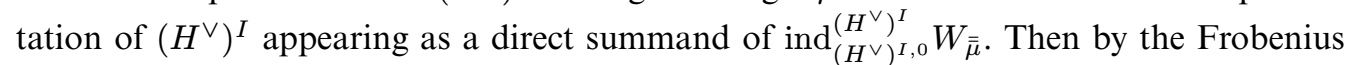

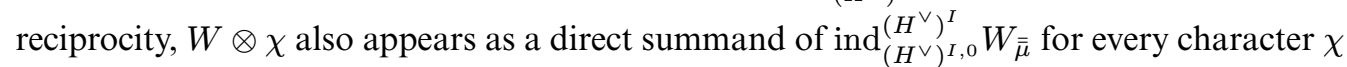
of $\left(H^{\vee}\right)^{I} /\left(H^{\vee}\right)^{I, 0}$, regarded as a representation of $\left(H^{\vee}\right)^{I}$. Then by Lemma 4.6 and counting the dimensions,

$$
\operatorname{ind}_{\left(H^{\vee}\right)^{I, 0}}^{\left(H^{\vee}\right)^{I}} \simeq \bigoplus_{\overline{\bar{\mu}}} \bigoplus_{\chi \in \operatorname{Rep}\left(\left(T^{\vee}\right)^{I} /\left(T^{\vee}\right)^{I, 0}\right)} W \otimes \chi,
$$

and the restriction $W$ to $\left(H^{\vee}\right)^{I, 0}$ is irreducible. Therefore the restriction $W$ to $\left(H^{\vee}\right)^{I, 0}$ is isomorphic to $W_{\overline{\bar{\mu}}}$. It is then clear that exactly one $(W \otimes \chi)$ appearing in the direct sum is an irreducible representation of $\left(H^{\vee}\right)^{I}$ of highest weight $\bar{\mu}$. This proves the existence. Then uniqueness is also clear because by the Frobenius reciprocity, every irreducible representation of $\left(H^{\vee}\right)^{I}$ appears as a direct summand in $\operatorname{ind}_{\left(H^{\vee}\right)^{I, 0}}^{\left(H^{\vee}\right)^{I}} W_{\overline{\bar{\mu}}}$ for some $\overline{\bar{\mu}} \in \mathbb{X}_{\bullet}(T)_{I} / \mathbb{X}_{\bullet}(T)_{I, \text { tor }}$. 
Now we finish the proof of the theorem. Let $\mu \in \mathbb{X}_{\bullet}(T)^{+}$be a lift of $\bar{\mu}$. Then, $\operatorname{Res}_{H^{\vee}}^{\left(H^{\vee}\right)^{I}}\left[V_{\mu}\right]=\left[W_{\bar{\mu}}\right]+\sum_{\bar{\lambda} \prec \bar{\mu}} c_{\bar{\lambda} \bar{\mu}}\left[W_{\bar{\lambda}}\right]$, where $[X]$ stands for the element in the $K$-group corresponding to $X$. Therefore,

$$
\operatorname{Res}_{H^{\vee}}^{\tilde{G}^{\vee}}\left[V_{\mu}\right]=\operatorname{Res}_{\left(H^{\vee}\right)^{I}}^{\tilde{G}^{\vee}}\left[W_{\bar{\mu}}\right]+\sum_{\bar{\lambda} \prec \bar{\mu}} c_{\bar{\lambda} \bar{\mu}} \operatorname{Res}_{\left(H^{\vee}\right)^{I}}^{\tilde{G}^{\vee}}\left[W_{\bar{\lambda}}\right] .
$$

On the other hand, for $\bar{\mu} \in \mathbb{X}_{\bullet}(T)_{I}^{+}$, the intersection cohomology sheaf $\mathrm{IC}_{\bar{\mu}} \in \mathscr{P}_{v}$ gives rise to an irreducible object $U_{\bar{\mu}}$ in $\operatorname{Rep}\left(\tilde{G}^{\vee}\right)$. By Lemma 2.6, we have

$$
\operatorname{Res}_{H^{\vee}}^{\tilde{G}^{\vee}}\left[V_{\mu}\right]=\left[U_{\bar{\mu}}\right]+\sum_{\bar{\lambda} \prec \bar{\mu}} d_{\bar{\lambda} \bar{\mu}}\left[U_{\bar{\lambda}}\right] .
$$

By induction on $\bar{\mu}$, one immediately obtains that

$$
\operatorname{Res}_{\left(H^{\vee}\right)^{I}}^{\tilde{G}^{\vee}}\left[W_{\bar{\mu}}\right]=U_{\bar{\mu}}+\sum_{\bar{\lambda} \prec \bar{\mu}} e_{\bar{\lambda} \bar{\mu}}\left[U_{\bar{\lambda}}\right] .
$$

Since $\left[W_{\bar{\mu}}\right]\left(\right.$ resp. $\left.\left[U_{\bar{\mu}}\right]\right)$ forms a $\mathbb{Z}$-basis of $K\left(\operatorname{Rep}\left(\left(H^{\vee}\right)^{I}\right)\right)\left(\operatorname{resp} . K\left(\operatorname{Rep}\left(\tilde{G}^{\vee}\right)\right)\right)$, this implies that $\operatorname{Res}_{\left(H^{\vee}\right)^{I}}^{\tilde{G}^{\vee}}$ is an isomorphism and therefore $\tilde{G}^{\vee}=\left(H^{\vee}\right)^{I}$.

The following table is a list of the group $\left(H^{\vee}\right)^{I}$ when $G$ over $k((\mathrm{t}))$ is absolutely simple and simply-connected, and non-split. Note that in this case, $I$ acts on the coweight lattice of $T_{H}$ by permutations of a basis and therefore $\mathbb{X}_{\bullet}(T)_{I}$ is torsion free. Therefore, by Lemma 4.6, $\left(H^{\vee}\right)^{I}$ is connected.

\begin{tabular}{|c|c|c|c|}
\hline$G$ & $H$ & $I$ & $\left(H^{\vee}\right)^{I}$ \\
\hline $\mathrm{SU}_{2 n-1}$ & $\mathrm{SL}_{2 n-1}, n \geq 2$ & $\mathbb{Z} / 2$ & $\mathrm{SO}_{2 n-1}$ \\
\hline $\mathrm{SU}_{2 n}$ & $\mathrm{SL}_{2 n}, n \geq 2$ & $\mathbb{Z} / 2$ & $\mathbf{P S p}_{2 n}$ \\
\hline${ }^{2} D_{n}$ & $\mathrm{Spin}_{2 n}, n \geq 4$ & $\mathbb{Z} / 2$ & $\mathrm{SO}_{2 n-1}$ \\
\hline${ }^{3} D_{4}$ & $\mathrm{Spin}_{8}$ & $\mathbb{Z} / 3$ & $G_{2}$ \\
\hline${ }^{2} E_{6}$ & $E_{6}$ & $\mathbb{Z} / 2$ & $F_{4}$ \\
\hline
\end{tabular}

Here $\mathbf{P S p} \mathrm{P}_{2 n}$ denotes the adjoint form of $\mathrm{Sp}_{2 n}$, and ${ }^{2} D_{n},{ }^{3} D_{4},{ }^{2} E_{6}$ are the quasi-split but nonsplit forms of $D_{n}$ and $E_{6}$.

Now, we switch to Theorem 0.2. Therefore, we will assume that $G$ is a quasi-split reductive group defined over the non-Archimedean local field $F=\mathbb{F}_{q}((\mathrm{t}))$ (we can in fact replace $\mathbb{F}_{q}$ by any other perfect field). Let $k=\overline{\mathbb{F}}_{q}$, and $\sigma$ be the Frobenius element in $\operatorname{Gal}\left(k / \mathbb{F}_{q}\right)$. Let $v \in \mathcal{B}(G, F)$ be a special vertex in the building which remains to be special when base change to $k((\mathrm{t}))$ (such a vertex is called very special, see $\S 6$ for more discussions). Let $\underline{G}_{v}$ be the special parahoric group scheme over $\mathbb{F}_{q}[[t]]$ corresponding to $v$ and $K_{v}=L^{+} \underline{G}_{v}$. Then the affine flag variety $\mathcal{F} \ell_{v}=L G / K_{v}$ is defined over $\mathbb{F}_{q}$ and when base change to $k, \mathcal{F} \ell_{v} \otimes k$ is the affine flag variety considered in the previous sections, and we have the Tannakian category $\mathscr{P}_{v}=\mathscr{P}_{K_{v} \otimes k}\left(\mathcal{F} \ell_{v} \otimes k\right)$ with a fiber functor $\mathrm{H}^{*}$.

As in Lemma A.5, there is an action of $\sigma$ on $\mathscr{P}_{v}$, and therefore an action of $\sigma$ on $\left(H^{\vee}\right)^{I}$. Following the notation as in the appendix, we denote this action by act ${ }^{\text {geom }}$. On the other hand, since there is a canonical pinning $\left(H^{\vee}, B_{H}^{\vee}, T_{H}^{\vee}, X^{\vee}\right)$, there is a canonical action of $\operatorname{Gal}\left(F^{s} / F\right)$ on $H^{\vee}$ by pinned automorphisms and therefore an action of $\sigma$ on $\left(H^{\vee}\right)^{I}$ by 
pinned automorphisms. We denote this action by act ${ }^{\text {alg }}$. As in the appendix, we denote cycl to be the cyclotomic character of $\operatorname{Gal}\left(k / \mathbb{F}_{q}\right)$, so that $\operatorname{cycl}(\sigma)=q$. Let

$$
\chi=\rho \circ \operatorname{cycl}: \operatorname{Gal}\left(k / \mathbb{F}_{q}\right) \rightarrow\left(H_{\mathrm{ad}}^{\vee}\right)^{I} .
$$

As in Proposition A.6, since the action of act ${ }^{\text {geom }}$ on $\left(H^{\vee}\right)^{I}$ fixes the cohomological grading and acts on $X^{\vee}$ via the cyclotomic character, we know that

$$
\operatorname{act}^{\text {geom }}=\operatorname{act}^{\text {alg }} \circ \mathrm{Ad}_{\chi} \text {, }
$$

and there is an isomorphism

$$
\left(H^{\vee}\right)^{I} \rtimes_{\text {act }}{ }^{\text {alg }} \operatorname{Gal}\left(k / \mathbb{F}_{q}\right) \rightarrow\left(H^{\vee}\right)^{I} \rtimes_{\text {actgeom }} \operatorname{Gal}\left(k / \mathbb{F}_{q}\right), \quad(g, \sigma) \mapsto\left(\operatorname{Ad}_{\chi(\sigma)^{-1}} g, \sigma\right) .
$$

Now regarding $\left(H^{\vee}\right)^{I} \rtimes_{\text {act alg }} \operatorname{Gal}\left(k / \mathbb{F}_{q}\right)$ as a pro-algebraic group over $\overline{\mathbb{Q}}_{\ell}$, as in the appendix, we have the category $\operatorname{Rep}\left(\left(H^{\vee}\right)^{I} \rtimes_{\text {act alg }} \operatorname{Gal}\left(k / \mathbb{F}_{q}\right)\right)$ of algebraic representations of $\left(H^{\vee}\right)^{I} \rtimes_{\text {actalg }} \operatorname{Gal}\left(k / \mathbb{F}_{q}\right)$. Now Theorem 0.2 follows from the same line as in the appendix.

\section{IC-stalks, $q$-analogy of the weight multiplicity, and the Lusztig-Kato polynomial}

Let $\bar{\mu} \in \mathbb{X}_{\bullet}(T)_{I}$ and $\mathcal{F} \in \mathscr{P}_{v}$. We determine the stalk cohomology $\mathcal{F}$ at the point $s_{\bar{\mu}}$. By abuse of notation, the inclusion map $s_{\bar{\mu}} \in \mathcal{F} \ell_{v}$ is still denoted by $s_{\bar{\mu}}$. It will be convenient to define

$$
\operatorname{Stalk}_{\bar{\mu}}(\mathcal{F})=s_{\bar{\mu}}^{*} \mathcal{F}[-(2 \rho, \bar{\mu})], \quad \operatorname{Costalk}_{\bar{\mu}}(\mathcal{F})=s_{\bar{\mu}} \mathcal{F}[(2 \rho, \bar{\mu})] .
$$

Let $X^{\vee}$ be the regular nilpotent element of $\operatorname{Lie}\left(H^{\vee}\right)^{I}$ given by the pinning. It defines an increasing filtration (the Brylinski-Kostant filtration) on any representation $V$ of $H^{\vee}$ or $\left(H^{\vee}\right)^{I}$,

$$
F_{i} V=\left(\operatorname{ker} X^{\vee}\right)^{i+1} .
$$

For $\bar{\mu} \in \mathbb{X}_{\bullet}(T)_{I}$, denote by $V(\bar{\mu})$ the $\bar{\mu}$-weight subspace of $V$, under the action of $\left(T_{H}^{\vee}\right)^{I}$. Then filtration (5.1) induces

$$
F_{i} V(\bar{\mu})=V(\bar{\mu}) \cap F_{i} V
$$

Let

$$
P_{\bar{\mu}}(V, q)=\sum \operatorname{gr}_{i}^{F} V(\bar{\mu}) q^{i}
$$

be the $q$-analogue weight multiplicity polynomial.

Theorem 5.1. - Let $\mathcal{F} \in \mathscr{P}_{v}$ and let $V=\mathrm{H}^{*}\left(\mathcal{F} \ell_{v}, \mathcal{F}\right)$ be the corresponding representation of $\left(H^{\vee}\right)^{I}$. Then

$$
P_{\bar{\mu}}(V, q)=\sum \operatorname{dim}^{-2 i}\left(\operatorname{Stalk}_{\bar{\mu}}(\mathcal{F})\right) q^{i}=\sum \operatorname{dim}^{2 i}\left(\operatorname{Costalk}_{\bar{\mu}}(\mathcal{F})\right) q^{i} .
$$

Observe that by the parity vanishing property of $\mathcal{F}, \operatorname{Stalk}_{\bar{\mu}} \mathcal{F}$ and $\operatorname{Costalk}_{\bar{\mu}}(\mathcal{F})$ only concentrate on even degrees.

In the split case, this is proved in [18, 4]. A more geometric proof is given by Ginzburg [10], which relies on the geometric Satake isomorphism and certain techniques of equivariant cohomology. We will follow Ginzburg's idea.

Let us give a quick review of equivariant cohomology (see [1] and [10, §8] for more details). Let $M$ be a variety with an action of a torus $A$. Let $\mathbb{B} A$ be the classifying space (stack) of $A$. Let $R_{A}=\mathrm{H}^{*}(\mathbb{B} A)$ and recall that $\operatorname{Spec} R_{A} \simeq \mathfrak{a}=\operatorname{Lie} A$. Let $t \in \mathfrak{a}$ be an element. We denote 
by $\kappa(\mathrm{t})$ the residue field of $t$ and let $\mathrm{H}_{t}:=\mathrm{H}_{A}^{*} \otimes_{R_{A}} \kappa(\mathrm{t})$. If $t=0$, this functor $\mathrm{H}_{0}$ inherits a canonical grading. For general $t$, this functor equips with a canonical filtration by

$$
\mathrm{H}_{t}^{\leq i}:=\operatorname{Im}\left(\sum_{j \leq i} \mathrm{H}_{A}^{j} \rightarrow \mathrm{H}_{t}\right)
$$

and there is a canonical isomorphism gr* $\mathrm{H}_{t} \simeq \mathrm{H}_{0}^{*}$. For every $\mathcal{F} \in D_{A}(M)$, there is a spectral sequence $E_{2}^{p, q}=\mathrm{H}^{p}\left(\mathbb{B} A, H^{q}(M, \mathcal{F})\right) \Rightarrow \mathrm{H}_{A}^{p+q}(M, \mathcal{F})$. If this spectral sequence degenerates at the $E_{2}$-term (which is always the case in the following discussion), then $\mathrm{H}_{0}^{*}(M, \mathcal{F}) \simeq$ $\mathrm{H}^{*}(M, \mathcal{F})$ and therefore we have a canonical isomorphism $\operatorname{gr~}_{t}(M, \mathcal{F}) \simeq \mathrm{H}^{*}(M, \mathcal{F})$.

Now assume that the action of $A$ on $M$ has only isolated fixed points $M^{A}$, and let $\eta \in \mathfrak{a}$ be the generic point. Then the localization theorem claims that there is an isomorphism

$$
\bigoplus_{x \in M^{A}} \mathrm{H}_{\eta}\left(i_{x}^{!} \mathcal{F}\right) \simeq \mathrm{H}_{\eta}(M, \mathcal{F}) \simeq \bigoplus_{x \in M^{A}} \mathrm{H}_{\eta}\left(i_{x}^{*} \mathcal{F}\right)
$$

where $i_{x}$ is the inclusion of the point $x$.

Next we review some results for split groups, which are essentially contained in [10]. However, our presentation here follows [29] closely. First, it is proved in [29, Lemma 2.2] that there is a canonical grading preserving isomorphism

$$
\mathrm{H}_{T_{H}}^{*} \simeq \mathrm{H}^{*} \otimes R_{T_{H}}: \mathrm{Sat}_{H} \rightarrow R_{T_{H}} \text {-Mod, }
$$

which endows $\mathrm{H}_{T_{H}}^{*}$ with a structure of tensor functors and defines a canonically trivialized $H^{\vee}$-torsor $\mathcal{E} \simeq H^{\vee} \times \mathfrak{t}_{H}$ on $\operatorname{Spec} R_{T_{H}}=\mathfrak{t}_{H}=:$ LieT $T_{H}$. In other words, the group scheme Aut ${ }^{\otimes} \mathrm{H}_{T_{H}}^{*}$ over $\mathfrak{t}_{H}$ of the tensor automorphism of this fiber functor, which a priori is an inner form of $H^{\vee}$, is canonically isomorphic to $H^{\vee} \times \mathfrak{t}_{H}$. In addition, the MV filtration and its canonical splitting extend in the equivariant setting [29, Lemma 2.2] and provide $T_{H}^{\vee} \times \mathfrak{t}_{H} \subset B_{H}^{\vee} \times \mathfrak{t}_{H} \subset H^{\vee} \times \mathfrak{t}_{H}$. Now, let $c^{T_{H}}(\mathscr{L}) \in \mathrm{H}_{T_{H}}^{2}\left(\mathrm{Gr}_{H}\right)$ denote the equivariant Chern class of $\mathscr{L}^{(3)}$. Then the action of $c^{T_{H}}(\mathscr{L})$ on $\mathrm{H}_{T_{H}}^{*}\left(\mathrm{Gr}_{H}, \mathcal{F}\right)$ for $\mathcal{F} \in \operatorname{Sat}_{H}$ can be identified with the action of an element

$$
e^{T_{H}} \in \Gamma\left(\mathfrak{t}_{H}, \operatorname{Lie}(\operatorname{ad} \mathcal{E})\right) .
$$

Since $\mathcal{E}$ is canonically trivialized, $e^{T_{H}}$ can be regarded as a map $\mathfrak{t}_{H} \rightarrow \mathfrak{h}^{\vee}$. Observe that $e^{T_{H}}$ is not the constant map $X^{\vee}$. In fact,

$$
e^{T_{H}}=X^{\vee}+h,
$$

where $h: \mathfrak{t}_{H} \rightarrow \mathfrak{t}_{H}^{\vee} \simeq\left(\mathfrak{t}_{H}\right)^{*}$ is given by a nondegenerate invariant bilinear form (cf. [29, Proposition 5.7]). In particular, $\left(H^{\vee}, B_{H}^{\vee}, T_{H}^{\vee}, e^{T_{H}}\right)$ is not a pinning over $\mathfrak{t}_{H}$.

The equivariant homology $\mathrm{H}_{*}^{T_{H}}\left(\mathrm{Gr}_{H}\right)$ is a commutative and cocommutative Hopf algebra and $J^{\vee}=\operatorname{SpecH}_{*}^{T_{H}}\left(\mathrm{Gr}_{H}\right)$ is a flat group scheme over $\mathfrak{t}_{H}$, acting on every $\mathrm{H}_{T_{H}}^{*}\left(\mathrm{Gr}_{H}, \mathcal{F}\right), \mathcal{F} \in \mathrm{Sat}_{H}$. By Tannakian formulism, this induces a map $\iota: J^{\vee} \rightarrow H^{\vee} \times \mathfrak{t}_{H}$. In [29], it is shown that this is a closed embedding, which identifies $J^{\vee}$ with the $\left(H^{\vee} \times \mathfrak{t}_{H}\right)^{e^{T_{H}}}$, the centralizer of $e^{T_{H}}$ in $H^{\vee} \times \mathfrak{t}_{H}$.

(3) By replacing $\mathscr{L}$ by a power of it, we can assume that $\mathscr{L}$ is $T_{H}$-equivariant. 
Let $\eta$ be the generic point of $\mathfrak{t}_{H}$. Then $J_{\eta}^{\vee}$ is indeed a torus in $H_{\eta}^{\vee}$ since $e^{T_{H}}(\eta) \in \mathfrak{h}_{\eta}^{\vee}$ is regular semisimple. Then localization theorem gives rise to an isomorphism of $J_{\eta}^{\vee}$-modules

$$
\bigoplus_{\mu \in \mathbb{X}_{\bullet}\left(T_{H}\right)} \mathrm{H}_{\eta}\left(s_{\mu}^{!} \mathcal{F}\right) \simeq \mathrm{H}_{\eta}\left(\mathrm{Gr}_{H}, \mathcal{F}\right)
$$

Following the idea of Ginzburg, we claim that this decomposition corresponds to the weight decomposition under $J_{\eta}^{\vee} \subset H_{\eta}^{\vee}$. First, let $B_{H}^{\vee} \rightarrow T_{H}^{\vee}$ be the natural projection. As is shown in [29], $J^{\vee} \subset B_{H}^{\vee} \times \mathfrak{t}_{H}$ and the composition $J^{\vee} \rightarrow B_{H}^{\vee} \times \mathfrak{t}_{H} \rightarrow T_{H}^{\vee} \times \mathfrak{t}_{H}$ is identified with the map (cf. Remark 3.4 of loc. cit.)

$$
R_{T_{H}}\left[\mathbb{X}_{\bullet}\left(T_{H}\right)\right] \simeq \bigoplus_{\mu \in \mathbb{X}_{\bullet}\left(T_{H}\right)} \mathrm{H}_{*}^{T_{H}}\left(s_{\mu}\right) \rightarrow \mathrm{H}_{*}^{T_{H}}\left(\mathrm{Gr}_{H}\right)
$$

Over $\eta$, this is an isomorphism and therefore we obtain a canonical isomorphism $J_{\eta}^{\vee} \simeq\left(T_{H}^{\vee}\right)_{\eta}$. In addition, the action of $J_{\eta}^{\vee}$ on $\mathrm{H}_{\eta, c}\left(S_{\mu}, \mathcal{F}\right)$ via $J_{\eta}^{\vee} \rightarrow\left(T_{H}^{\vee}\right)_{\eta}$ is identified with the natural action of $J_{\eta}^{\vee}$ on $\mathrm{H}_{\eta}\left(s_{\mu}^{!} \mathcal{F}\right) \simeq \mathrm{H}_{\eta, c}\left(S_{\mu}, \mathcal{F}\right)$. Therefore, we obtain the following proposition, originally proved by Ginzburg by another method.

Proposition 5.2. - Let $V \in \operatorname{Rep}\left(H^{\vee}\right)$ and $\phi(V) \in$ Sat $_{H}$ be the corresponding sheaf ( see (2.1)). Under the identification of the weight lattice of $J_{\eta}^{\vee}$ with $\mathbb{X}_{\bullet}\left(T_{H}^{\vee}\right)$ via the canonical isomorphism $J_{\eta}^{\vee} \rightarrow\left(T_{H}^{\vee}\right)_{\eta}$, the direct summand $\mathrm{H}_{\eta}\left(s_{\mu}^{!} \phi(V)\right) \subset \mathrm{H}_{\eta}\left(\mathrm{Gr}_{H}, \phi(V)\right)$ corresponds to the weight subspace $V(\mu) \subset V$ for $J_{\eta}^{\vee}$.

REMARK 5.3. - Let us observe that the localization isomorphism (5.5) holds over $\mathfrak{t}_{H}$ after we remove all root hyperplanes. This is because for every $T_{H}$-invariant finite dimensional closed subvariety $Z \subset \mathrm{Gr}_{H}$, there are only finitely many 1 -dimensional $T_{H}$-orbits in $Z$, and $T_{H}$ acts on these orbits via rotations determined by roots. Therefore, in all the discussions above, we can replace the generic point $\eta$ by any (closed) point in $\mathfrak{t}_{H} \backslash\left\{\tilde{a}=0, \tilde{a} \in \Phi_{H}\right\}$, where $\Phi_{H}$ is the set of roots of $H$.

Now let $t$ be a closed point on $\mathfrak{t}_{H}$ such that

$$
h(\mathrm{t})=2 \rho
$$

so that $e^{T_{H}}(\mathrm{t})=X^{\vee}+h(\mathrm{t})=X^{\vee}+2 \rho$. According to [29, Proposition 5.7], such point exists (unique up to adding an element in the center $\mathfrak{z}(\mathfrak{h})$ of $\mathfrak{h}$ ) and does not belong to any root hyperplanes. Therefore, the localization isomorphism (5.5) holds for $\mathrm{H}_{t}$ by the above remark. From now on, we will always choose the point $t$ satisfying (5.6).

Recall that under the geometric Satake isomorphism $\mathrm{H}^{*}: \operatorname{Sat}_{H} \simeq \operatorname{Rep}\left(H^{\vee}\right)$, the natural grading on the cohomology functor corresponds to the principal grading on representations of $H^{\vee}$. More precisely, consider the cocharacter $2 \rho: \mathbb{G}_{m} \rightarrow T_{H}^{\vee} \subset H^{\vee}$. Then the grading on the cohomology functor corresponds to the grading given by $2 \rho$ on the representations. This follows from the fact that $\mathrm{H}_{c}^{*}\left(S_{\mu}, \mathcal{F}\right)$ is nonzero only in degree $(2 \rho, \mu)$. Now it is clear from (5.4) that for the closed point $t \in \mathfrak{t}_{H}$, the filtration $\mathrm{H}_{t}^{\leq i}$ corresponds to the increasing filtration on the representations associated to the gradings given by $2 \rho$. For $i \in \mathbb{Z}$, let

$$
\mathbb{X}_{\bullet}\left(T_{H}\right)_{i}=\left\{\mu \in \mathbb{X}_{\bullet}\left(T_{H}\right) \mid(2 \rho, \mu)=i\right\} .
$$

$4^{\text {e }}$ SÉRIE - TOME $48-2015$ - No 2 
Let $V$ be a representation of $H^{\vee}$. Denote

$$
V(\mathrm{i})=\sum_{\mu \in \mathbb{X} \cdot\left(T_{H}\right)_{i}} V(\mu)
$$

where $V(\mu)$ is the $\mu$-weight space of $J_{t}^{\vee}$. Let us identify $\mathrm{H}_{t}\left(\mathrm{Gr}_{H}, \phi(V)\right)$ with $V$ canonically, so that $\mathrm{H}_{t}\left(s_{\mu}^{*} \phi(V)\right)$ is identified with $V(\mu)$ by Proposition 5.2. Then we have the following proposition.

Proposition 5.4. - Let $t$ be as in (5.6). Write $\mathrm{H}_{t}=\mathrm{H}_{t}\left(\mathrm{Gr}_{H}, \varnothing(V)\right)$ for simplicity. Then for any $m \in \mathbb{Z}$,

$$
\mathrm{H}_{t}^{\leq 2 i+m} \cap \bigoplus_{\mu \in \mathbb{X} \bullet(T)_{m}} \mathrm{H}_{t}\left(s_{\mu}^{!} \phi(V)\right)=\mathrm{H}_{t}^{\leq 2 i+1+m} \cap \bigoplus_{\mu \in \mathbb{X} \bullet(T)_{m}} \mathrm{H}_{t}\left(s_{\mu}^{!} \phi(V)\right)=F_{i} V \cap V(\mathrm{~m}),
$$

where $F_{i} V$ is defined as in (5.1).

Proof. - Let $n$ be the unique element in $U_{H}^{\vee}$ such that $\operatorname{Ad}_{n}\left(X^{\vee}+2 \rho\right)=2 \rho$. Then the canonical isomorphism $J_{t}^{\vee} \simeq T_{H}^{\vee}$ is given by $\operatorname{Ad}_{n}: J_{t}^{\vee} \rightarrow T_{H}^{\vee}$. The proposition clearly follows from the following purely representation theoretical lemma.

Lemma 5.5. - Let $V$ be a representation of $H^{\vee}$. Let

$$
V=\sum V^{1}(\mathrm{i}), \quad V=\sum V^{2}(\mathrm{i})
$$

be two gradings on $V$, given by the cocharacters $2 \rho: \mathbb{G}_{m} \rightarrow H^{\vee}$ and $\operatorname{Ad}_{n^{-1}} 2 \rho: \mathbb{G}_{m} \rightarrow H^{\vee}$ respectively. Let $F_{\bullet}^{1} V$ and $F_{\bullet}^{2} V$ be two filtrations on $V$ given by

$$
F_{i}^{1} V=\sum_{j \leq i} V^{1}(\mathrm{j}), \quad F_{i}^{2} V=\left(\operatorname{ker} X^{\vee}\right)^{i+1}
$$

Then for any $m \in \mathbb{Z}, V^{2}(\mathrm{~m}) \cap F_{2 i+m}^{1} V=V^{2}(\mathrm{~m}) \cap F_{2 i+1+m}^{1} V=V^{2}(\mathrm{~m}) \cap F_{i}^{2} V$.

Proof. - Let $Y^{\vee} \in \mathfrak{h}^{\vee}$ so that $\left\{X^{\vee}, 2 \rho, Y^{\vee}\right\}$ forms an $\mathfrak{s l}_{2}$-triple. Then the lemma is purely a statement about this $\mathfrak{s l}_{2}$ and can be checked easily by direct calculation.

This finishes the discussion for split groups. Now let $G$ be as before and $A$ be its maximal split torus. By the isomorphism (1.1), we can regard $A$ as a subtorus of $T_{H}$. Note that we can restrict everything discussed above to $A \subset T_{H}$. In particular, we can choose the point $t \in \mathfrak{a}$ such that $h(\mathrm{t})=2 \rho$. This is because $h: \mathfrak{t}_{H} \rightarrow \mathfrak{t}_{H}^{\vee}$ is equivariant under the automorphisms of the based root datum and $2 \rho$ is a fixed point under these automorphisms.

We begin to prove the theorem. Observe that it is enough to prove the theorem for objects in $\mathscr{P}_{v}$ of the form $Z(\mathcal{F})$, where $\mathscr{F} \in \operatorname{Sat}_{H}$. Indeed, both maps $\mathscr{P}_{v} \rightarrow \mathbb{Z}[q]$ given by $\mathcal{F} \mapsto P_{\bar{\mu}}\left(\mathrm{H}^{*}(\mathcal{F}), q\right)$ and $\mathcal{F} \mapsto \sum \operatorname{dim}^{2 i}\left(\operatorname{Costalk}_{\bar{\mu}}(\mathcal{F})\right) q^{i}$ factor through the Grothendieck group, and as observed in the proof of Theorem 0.1 in Section 4, the objects of the form $Z(\mathcal{F}), \mathcal{F} \in \operatorname{Sat}_{H}$ generate the Grothendieck group of $\mathscr{P}_{v}$.

Recall that the maximal torus $A \subset G$ extends naturally to a split torus over $\theta$ and $A_{\vartheta} \subset \underline{G}_{v}$. Therefore, we can regard $A$ as a subtorus of $K_{v}$, as $A$ is a natural subgroup of $L^{+} A_{\vartheta}$ consisting of "constant" elements. The set of fixed points of the action of $A$ on $\mathcal{F} \ell_{v}$ are exactly $\left\{s_{\bar{\mu}} \mid \bar{\mu} \in \mathbb{X}_{\bullet}(T)_{I}\right\}$. This will be clear if we regard $L G$ as a Kac-Moody group and 
$A$ as its maximal torus. We consider the $A$-equivariant cohomology $\mathrm{H}_{A}^{*}: \mathscr{P}_{v} \rightarrow R_{A}$-Mod. Since the nearby cycles commute with proper base change, we have a canonical isomorphism

$$
\mathrm{H}_{A}^{*} \circ Z \simeq \mathrm{H}_{A}^{*}: \operatorname{Sat}_{H} \rightarrow R_{A} \text {-Mod. }
$$

Indeed, when fixing a cohomological degree, we can replace $\mathbb{B} A$ by $\left(\mathbb{P}^{n}\right)^{\text {rk } A}$ for $n$ large enough, and consider the nearby cycle functors for the family $\widetilde{\mathrm{Gr}}_{\mathscr{G}} \times{ }^{A}\left(\mathbb{P}^{n}\right)^{\mathrm{rk} A}$. The claim then is clear.

REMARK 5.6. - One should be able to argue as defining the tensor structure of $\mathrm{H}^{*}$, that there is a canonical isomorphism

$$
\mathrm{H}_{A}^{*} \simeq \mathrm{H}^{*} \otimes R_{A}: \mathscr{P}_{v} \rightarrow R_{A} \text {-Mod, }
$$

which endows $\mathrm{H}_{A}^{*}$ with a fiber functor structure, and the corresponding $\left(H^{\vee}\right)^{I}$ torsor on $\operatorname{Spec} R_{A}=\mathfrak{a}=: \operatorname{Lie} A$ is canonically trivialized. However, we did not investigate this.

Let $p: \mathbb{X}_{\bullet}(T) \rightarrow \mathbb{X}_{\bullet}(T)_{I}$ be the projection. Let $\eta$ be the generic point of $\mathfrak{a}$. Now, the key observation is

Lemma 5.7. - Under the canonical isomorphism $\mathrm{H}_{\eta}\left(\mathcal{F} \ell_{v}, Z(\mathcal{F})\right) \simeq \mathrm{H}_{\eta}\left(\mathrm{Gr}_{H}, \mathcal{F}\right)$, the direct summand $\mathrm{H}_{\eta}^{*}\left(s_{\bar{\mu}}^{!} \mathcal{Z}(\mathcal{F})\right)$ corresponds to $\bigoplus_{\mu \in p^{-1}(\bar{\mu})} \mathrm{H}_{\eta}^{*}\left(s_{\mu}^{!} \mathcal{F}\right)$.

Proof. - Recall that if $f: \chi \rightarrow \mathcal{Y}$ is a morphism of varieties over $\mathbb{A}^{1}$, then there are always the natural maps $f^{*} \Psi_{y} \rightarrow \Psi_{\chi} f^{*}$ and $\Psi_{y} f_{*} \rightarrow f_{*} \Psi_{\chi}$ (see SGA 7 Exposé XIII, (2.1.7.1) (2.1.7.2)). In addition, these two maps fit into the following commutative diagram

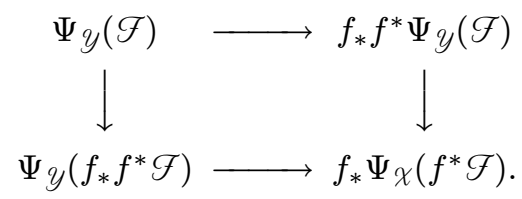

Now let $\mu \in \mathbb{X}_{\bullet}\left(T_{H}\right)$ and apply this remark to $s_{\mu}: \mathbb{A}^{1} \rightarrow \widetilde{\operatorname{Gr}}_{g}$ as defined in the proof of Lemma 2.6. By taking the cohomology $\mathrm{H}_{A}^{*}$, we obtain, for any $\mathcal{F} \in \mathrm{Sat}_{H}$, the following commutative diagram

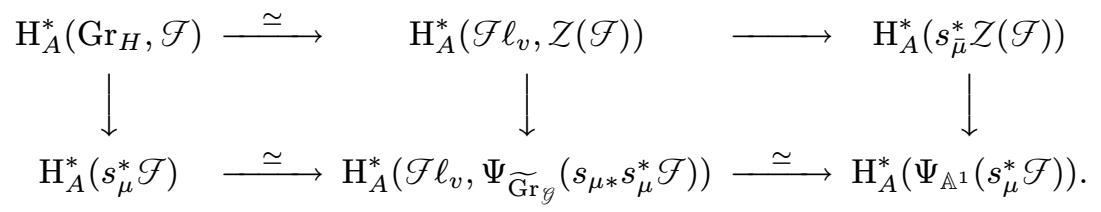

In other words, the composition $\mathrm{H}_{A}^{*}\left(\mathcal{F} \ell_{v}, Z(\mathcal{F})\right) \rightarrow \mathrm{H}_{A}^{*}\left(\mathrm{Gr}_{H}, \mathcal{F}\right) \rightarrow \mathrm{H}_{A}^{*}\left(s_{\mu}^{*} \mathcal{F}\right)$ factors as $\mathrm{H}_{A}^{*}\left(\mathcal{F} \ell_{v}, \mathcal{Z}(\mathcal{F})\right) \rightarrow \mathrm{H}_{A}^{*}\left(s_{\bar{\mu}}^{*} \mathcal{Z}(\mathcal{F})\right) \rightarrow \mathrm{H}_{A}^{*}\left(s_{\mu}^{*} \mathcal{F}\right)$. On the other hand, by the localization theorem, over the generic $\eta$ of $\mathfrak{a}$, we have

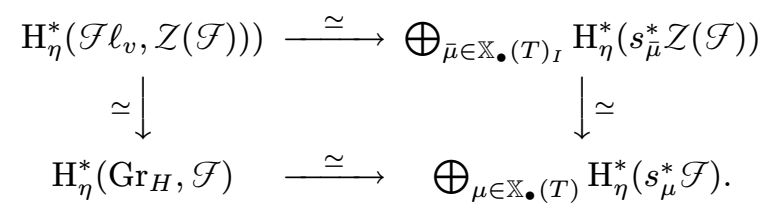

Observe that in the localization Theorem (5.3), for $x, y \in M^{A}$ and $x \neq y$, the composition $\mathrm{H}_{\eta}\left(i_{x}^{!} \mathcal{F}\right) \rightarrow \mathrm{H}_{\eta}\left(i_{y}^{*} \mathcal{F}\right)$ is zero. Therefore, the lemma follows from (5.7) and (5.8). 
As a result, by Proposition 5.2, if $\mathcal{F}=\varnothing(V)$, this direct summand can be further identified with $\bigoplus_{\mu \in p^{-1}(\bar{\mu})} V(\mu)$, the weight subspaces under $J_{\eta}^{\vee}$. In addition, by Proposition 5.4,

$$
\mathrm{H}_{t}^{\leq 2 i+(2 \rho, \bar{\mu}))} \cap \mathrm{H}_{t}\left(s_{\bar{\mu}}^{!} Z(\phi(V))\right)=\mathrm{H}_{t}^{\leq 2 i+1+(2 \rho, \bar{\mu}))} \cap \mathrm{H}_{t}\left(s_{\bar{\mu}} Z(\phi(V))\right)=F_{i} V \cap \bigoplus_{\mu \in p^{-1}(\bar{\mu})} V(\mu),
$$

where we write $\mathrm{H}_{t}=\mathrm{H}_{t}\left(\mathcal{F} \ell_{v}, Z(\varnothing(V))\right)$ for brevity. Therefore, to finish the proof of the theorem, it remains to show that

Lemma 5.8. - Let $\mathcal{F} \in \mathscr{P}_{v}$. Then the canonical map

$$
\mathrm{H}_{A}^{*}\left(s_{\bar{\mu}} \mathcal{F}\right) \rightarrow \mathrm{H}_{A}^{*}(\mathcal{F})
$$

is a splitting injective map of free $R_{A}$-modules. Therefore,

$$
\mathrm{H}_{\bar{t}}^{\leq i}\left(\mathcal{F} \ell_{v}, \mathcal{Z}(\phi(V))\right) \cap \mathrm{H}_{t}\left(s_{\bar{\mu}}^{!} \mathcal{Z}(\varnothing(V))\right)=\mathrm{H}_{\bar{t}}^{\leq i}\left(s_{\bar{\mu}}^{!} \mathcal{Z}(\phi(V))\right) .
$$

These are general facts about flag varieties for Kac-Moody groups. The basic geometric fact behind this proposition is that the "big open cell" of the flag variety contracts to a point under certain $\mathbb{G}_{m}$-action. Then the statement follows using an argument with weights (cf. [10]). We here reproduce the proof for completeness.

Proof. - Without loss of generality, we can assume that $\mathcal{F}$ is an intersection cohomology complex. First, we claim that it is enough to prove a dual statement: the map

$$
\mathrm{H}_{A}^{*}(\mathcal{F}) \rightarrow \mathrm{H}_{A}^{*}\left(s_{\bar{\mu}}^{*} \mathcal{F}\right)
$$

is surjective. To see this, recall that since each of $\mathrm{H}^{*}(\mathcal{F}), s_{\bar{\mu}}^{*} \mathcal{F}, s_{\bar{\mu}}^{!} \mathcal{F}$ concentrates in cohomological degrees of the same parity, the spectral sequence calculating the $A$-equivariant cohomology degenerates at the $E_{2}$-term, which implies that all $\mathrm{H}_{A}^{*}(\mathcal{F}), \mathrm{H}_{A}^{*}\left(s_{\bar{\mu}}^{*} \mathcal{F}\right), \mathrm{H}_{A}^{*}\left(s_{\bar{\mu}}^{!} \mathcal{F}\right)$ are finite free $R_{A}$-modules. Then taking $\operatorname{Hom}\left(-, R_{A}\right)$ interchanges (5.9) and (5.10).

Since $\mathcal{F} \ell_{v}$ is the flag variety of certain Kac-Moody group (cf. [22, Section 9.h]), for every $s_{\bar{\mu}}$, there is a $\mathbb{G}_{m}$-action on $\mathcal{F} \ell_{v}$, contracting an open neighborhood of $s_{\bar{\mu}}$ in $\mathcal{F} \ell_{v}$ to $s_{\bar{\mu}}$. In addition, this $\mathbb{G}_{m}$-action stabilizes every Schubert cell $\mathscr{S}^{\circ} \ell_{\bar{\lambda}}^{s}$, and commutes with the action of $A$ on $\mathcal{F} \ell_{v}$. Denote this open neighborhood by $j: U_{\bar{\mu}} \hookrightarrow \mathcal{F} \ell_{v}$. Then $U_{\bar{\mu}}$ is an inductive limit of affine spaces. Indeed, $U_{\overline{0}}$ is just the big open cell in the flag variety, and $U_{\bar{\mu}}$ is the translate $U_{\overline{0}}$ via $s_{\bar{\mu}}$ (lifted to an element in $T(F)$ ).

Now we can assume that our group is defined over $\mathbb{F}_{q}((\mathrm{t}))$ and splits over a totally ramified extension. All the discussion above remains unchanged in this setting. Recall that we denote $\mathscr{P}_{v}^{0}$ to be the semisimple $K_{v}$-equivariant perverse sheaves on $\mathcal{F} \ell_{v}$, pure of weight zero. It is well-known that for every object $\mathcal{F}$ in $\mathscr{P}_{v}, \mathrm{H}_{A}^{*}\left(s_{\bar{\mu}}^{*} \mathcal{F}\right)$ is pure of weight zero (i.e., $\mathrm{H}_{A}^{i}\left(s_{\bar{\mu}}^{!} \mathcal{F}\right)$ is pure of weight $i$ ), essentially due to the existence of Demazure resolutions. To show (5.10) is surjective, we decompose this map into

$$
\mathrm{H}_{A}^{*}(\mathcal{F}) \rightarrow \mathrm{H}_{A}^{*}\left(U_{\bar{\mu}}, j^{*} \mathcal{F}\right) \rightarrow \mathrm{H}_{A}^{*}\left(s_{\bar{\mu}}^{*} \mathcal{F}\right) .
$$

It is well-known that the second map is an isomorphism since $j^{*} \mathcal{F}$ is equivariant under this $\mathbb{G}_{m}$-action, which contracts $\left(U_{\bar{\mu}} \cap \operatorname{Supp} \mathcal{F}\right)$ to $s_{\bar{\mu}}$. In particular, $\mathrm{H}_{A}^{*}\left(U_{\bar{\mu}}, j^{*} \mathcal{F}\right)$ is pure of weight zero. Therefore, it is enough to show that the first map is surjective. Denote $i: Z=\mathcal{F} \ell_{v} \backslash U_{\bar{\mu}} \hookrightarrow \mathcal{F} \ell_{v}$ to be the complement. Then we have the distinguished triangle

$$
i_{*} i^{!} \mathcal{F} \rightarrow \mathcal{F} \rightarrow j_{*} j^{*} \mathcal{F} \rightarrow
$$


and therefore

$$
0 \rightarrow \mathrm{H}_{A}^{*}(i ! \mathcal{F}) \rightarrow \mathrm{H}_{A}^{*}(\mathcal{F}) \rightarrow \mathrm{H}_{A}^{*}\left(U_{\bar{\mu}}, j^{*} \mathcal{F}\right) \rightarrow 0 .
$$

The last map is surjective because the weights of $\mathrm{H}_{A}^{*}\left(i^{!} \mathscr{F}\right)$ are $\geq 0$.

\section{The Langlands parameter}

In this section, we briefly discuss the Langlands parameters for smooth "spherical" representations of a quasi-split $p$-adic group. The parameters themselves can be described easily, and they will be used when we discuss the Frobenius trace of nearby cycles for certain unitary Shimura varieties.

We will assume that $F$ is a non-Archimedean local field with finite residue field and that $G$ is a connected reductive group over $F$. First, we generalize the hyperspecial vertex of an unramified group as follows. Recall that by [27], the building of $G(F)$ can be embedded into the building of $G(L)$, where $L$ is the completion of a maximal unramified extension of $F$.

Definition 6.1. - A special vertex $v$ of $G$ is called geometrically special (or very special) if it remains special in $G_{L}$. The parahoric subgroup of $G$ corresponding to a geometrically special vertex is called a geometrically special (or very special) parahoric subgroup of $G$.

Clearly, if $G$ is an unramified group, then very special vertices of $G$ are the same as hyperspecial vertices of $G$.

Lemma 6.1. - A very special vertex of $G$ exists if and only if $G$ is quasi-split over $F$.

Proof. - Assume that $G$ is quasi-split. Then the existence of such points follows exactly by the same argument as in $[27,1.10 .2]$. We prove the converse. Let $v$ be a very special point. Choose a maximal $F$-split torus $A$ of $G$ such that the corresponding apartment $A(G, A, F)$ containing $v$. Let $S$ be a maximal $L$-split torus defined over $F$ and containing $A$. We identify the apartment $A\left(G_{L}, S_{L}, L\right)$ with $\mathbb{X}_{\bullet}(S) \otimes \mathbb{R}$ by $v$. As $v$ is special, there is a bijection between the finite Weyl chambers for $\left(G_{L}, S_{L}\right)$ and the affine Weyl chambers (or called alcove) with $v$ as a vertex, and this bijection is compatible with the action of $\operatorname{Gal}(L / F)$. To show that $G$ is quasi-split, it is enough to find an $L$-rational Borel containing $S$ stable under $\operatorname{Gal}(L / F)$, which is equivalent to finding a finite Weyl chamber in $\mathbb{X}_{\bullet}(S) \otimes \mathbb{R}$, stable under $\operatorname{Gal}(L / F)$. Therefore, it enough to show that among all alcoves with $v$ as a vertex, there is one stable under $\operatorname{Gal}(L / F)$. But as it is known, one of such alcoves intersects with $A(G, A, F)$ (since every reductive group over $F$ is residually quasi-split, see [27, §1.10]), which is stable under $\operatorname{Gal}(L / F)$.

In fact, by checking the classification of central isogeny classes of quasi-simple, absolutely simple reductive group over $F$ as in [27, §4], we find that if $G$ is quasi-split, then every special vertex of $G$ is very special except the following case: up to central isogeny, $G$ is an unramified odd unitary group. Then there are two special vertices in its relative local Dynkin diagram, only one of which is hyperspecial. To prove this assertion, one uses the following observation: Using the notation as in loc. cit., a vertex $v$ in $\Delta$ (the relative local Dynkin diagram of $G$ ) is very special if and only if the corresponding $\operatorname{Gal}(L / F)$-orbit $O(\mathrm{v}) \subset \Delta_{1}$ (the absolute local Dynkin diagram of $G$ ) consists of one point, which is special in $\Delta_{1}$.

4 e SÉRIE - TOME $48-2015$ - No 2 
Next we turn to representations.

Definition 6.2. - We call an irreducible smooth representation $V$ of $G$ spherical, if there is some $v \in V, v \neq 0$, which is fixed by some very special parahoric subgroup of $G$.

Remark 6.2. - Again, one could try to define spherical representations of $G$ as those with a vector fixed by some special maximal compact subgroup of $G$. However, from the point of view of Langlands parameters discussed below, this is not correct.

Clearly, if $G$ is unramified, spherical representations are those usually called unramified representations. For the unramified representations, the description the associated Langlands parameters is well-known (for example, see [3, Chapter II]). Let us explain the Langlands parameters of spherical representations for quasi-split ramified groups.

Following the notation in the previous sections, we denote by $H^{\vee}$ its dual group in the sense of Langlands defined over $\mathbb{C}$, i.e., the root datum is dual to the root datum of $G$. Let us equip $H^{\vee}$ with a pinning $\left(H^{\vee}, B^{\vee}, T^{\vee}, X^{\vee}\right)^{(4)}$. Then $\operatorname{Gal}\left(F^{s} / F\right)$ acts on $H^{\vee}$ via pinned automorphisms, which we denote by act ${ }^{\text {alg }}$, and we can form the Langlands dual group of $G$ as

$$
{ }^{L} G^{\text {alg }}=H^{\vee} \rtimes_{\text {act alg }} \operatorname{Gal}\left(F^{s} / F\right) .
$$

Let $W_{F}$ be the Weil group of $F$. A Langlands parameter is a continuous homomorphism (up to conjugation by $H^{\vee}$ ) $\rho: W_{F} \rightarrow{ }^{L} G^{\text {alg }}$, such that its composition with the canonical projection ${ }^{L} G^{\text {alg }} \rightarrow \operatorname{Gal}\left(F^{s} / F\right)$ is the natural inclusion $W_{F} \rightarrow \operatorname{Gal}\left(F^{s} / F\right)$ and $\rho\left(W_{F}\right)$ consists of semisimple elements of ${ }^{L} G^{\text {alg }}$ (see [3, 8.2] for the unexplained terminology).

We write $\rho(\gamma)=\left(\rho_{1}(\gamma), \gamma\right)$ for $\gamma \in W_{F}$, where $\rho_{1}$ is a map from $W_{F}$ to $H^{\vee}$.

Definition 6.3. - A "spherical" parameter (or Langlands-Satake parameter) is a Langlands parameter $\rho$ which can be conjugated to the form $\rho(\gamma)=(1, \gamma)$ for $\gamma$ in the inertial group $I$.

Let $\left(H^{\vee}\right)^{I}$ be the $I$-fixed point subgroup of $H^{\vee}$ (which could be non-connected according to Remark 4.4). Then $\operatorname{Gal}\left(F^{s} / F\right) / I$ acts on $\left(H^{\vee}\right)^{I}$ through a finite cyclic group $\langle\sigma\rangle$, where $\sigma \in \operatorname{Gal}\left(F^{s} / F\right) / I$ is the Frobenius element.

Lemma 6.3. - "Spherical" Langlands parameters $\rho: W_{F} \rightarrow{ }^{L} G^{\text {alg }}$ are in one-to-one correspondence to semi-simple elements in $\left(H^{\vee}\right)^{I} \times \sigma \subset\left(H^{\vee}\right)^{I} \rtimes_{\text {actalg }}\langle\sigma\rangle$ up to conjugacy by $\left(H^{\vee}\right)^{I}$.

We denote the set of semi-simple elements in $\left(H^{\vee}\right)^{I} \times \sigma$ by $\left(\left(H^{\vee}\right)^{I} \times \sigma\right)_{s s}$.

Proof. - First, observe that elements $h \in H^{\vee}$ satisfy that $(h, 1)(1, \gamma)\left(h^{-1}, 1\right)=(1, \gamma)$ for all $\gamma \in I$ if and only if $h \in\left(H^{\vee}\right)^{I}$.

Let $\Phi$ be a lift of the Frobenius element to $W_{F}$. Then $\rho$ is uniquely determined by $\rho_{1}(\Phi)$, which is a semi-simple element in $H^{\vee}$. Let $\gamma \in I$. Then $\left(1, \Phi \gamma \Phi^{-1}\right)=\rho\left(\Phi \gamma \Phi^{-1}\right)=$ $\left(\rho_{1}(\Phi), \Phi\right)(1, \gamma)\left(\rho_{1}\left(\Phi^{-1}\right), \Phi^{-1}\right)$ implies that $\rho_{1}(\Phi)$ is invariant under $I$. Conversely, if $g \in H^{\vee} \rtimes_{\text {actalg }} I$, then the formulas $\rho_{1}(I)=1, \rho_{1}(\Phi)=g$ define $\rho$.

(4) In fact, by the construction of the appendix, there is a canonical pinned of $H^{\vee}$ provided by the geometric Satake correspondence. 
Let $A$ be a maximal $F$-split torus and let $T$ be the centralizer of $A$, which is a maximal torus of $G$. Let $W_{0}=W(G, A)$ be the Weyl group. As explained in [11, Remark 9], we can identify $W_{0}$ with the $\sigma$ invariants of the Weyl group $W\left(\left(H^{\vee}\right)^{I},\left(T^{\vee}\right)^{I}\right)$ (observe that the latter group was denoted by $W_{0}$ in Section 4$)$, and let $N_{0}$ be the inverse image of $W_{0}$ inside the normalizer of $\left(T^{\vee}\right)^{I}$ in $\left(H^{\vee}\right)^{I}$. Let $\operatorname{Rep}\left(\left(H^{\vee}\right)^{I} \rtimes_{\text {actalg }}\langle\sigma\rangle\right)$ be a category of algebraic representations of $\left(H^{\vee}\right)^{I} \rtimes_{\text {act }}$ alg $\langle\sigma\rangle$.

For every $W \in \operatorname{Rep}\left(\left(H^{\vee}\right)^{I} \rtimes_{\text {act }}\right.$ alg $\left.\langle\sigma\rangle\right)$, by restriction of its character to $\left(H^{\vee}\right)^{I} \times \sigma$, we obtain a function $\operatorname{ch}_{W}$ on $\left(H^{\vee}\right)^{I} \times \sigma$. We denote by R the algebra of functions on $\left(H^{\vee}\right)^{I} \times \sigma$, generated by all $\mathrm{ch}_{W}$. We can adapt the proofs in [3, 6.4-6.7] to the group $\left(H^{\vee}\right)^{I} \rtimes\langle\sigma\rangle$, and obtain

Proposition 6.4. - (i) The natural map $\mathbb{X}_{\bullet}(T)_{I}^{\sigma} \subset \mathbb{X}_{\bullet}(T)_{I}$ induces an isomorphism

$$
\alpha:\left(T^{\vee}\right)^{I} \rtimes \sigma / \operatorname{Int} N_{0} \simeq \operatorname{Spec} \mathbb{C}\left[\mathbb{X}_{\bullet}(T)_{I}^{\sigma}\right]^{W_{0}} .
$$

(ii) The natural map $\left(T^{\vee}\right)^{I} \rtimes \sigma \rightarrow\left(H^{\vee}\right)^{I} \rtimes \sigma$ induces an isomorphism

$$
\beta:\left(T^{\vee}\right)^{I} \rtimes \sigma / \operatorname{Int} N_{0} \simeq\left(\left(H^{\vee}\right)^{I} \rtimes \sigma\right)_{s s} / \operatorname{Int}\left(\left(H^{\vee}\right)^{I}\right) .
$$

(iii) The composition $\beta \alpha^{-1}: \operatorname{Spec} \mathbb{C}\left[\mathbb{X} \bullet(T)_{I}^{\sigma}\right]^{W_{0}} \rightarrow\left(\left(H^{\vee}\right)^{I} \times \sigma\right)_{\text {ss }} / \operatorname{Int}\left(\left(H^{\vee}\right)^{I}\right)$ induces an isomorphism

$$
\mathbb{C}\left[\mathbb{X}_{\bullet}(T)_{I}^{\sigma}\right]^{W_{0}} \simeq \mathrm{R}
$$

as functions on $\left(\left(H^{\vee}\right)^{I} \times \sigma\right)_{s s} / \operatorname{Int}\left(H^{\vee}\right)^{I}$.

Therefore, the set of spherical Langlands parameters can be identified with the set of all characters of $R$. Namely if $\rho$ is a spherical parameter, then the corresponding character $\chi_{\rho}: R \rightarrow \mathbb{C}$ is given by

$$
\chi_{\rho}\left(\operatorname{ch}_{W}\right)=\operatorname{tr}(\rho(\Phi), W),
$$

where we assume (after conjugation) that $\rho(\Phi) \in\left(\left(H^{\vee}\right)^{I} \times \sigma\right)_{s s}$, and $\in\left(H^{\vee}\right)^{I} \rtimes\langle\sigma\rangle$.

Now let us explain how to attach to a spherical representation its spherical parameter. Let $\pi$ be a spherical representation of $G$, such that $\pi^{K_{v}} \neq 0$ for a very special parahoric subgroup $K_{v} \subset G(F)$. Therefore, $\pi$ determines a character $\chi_{\pi}$ of $C_{c}\left(K_{v} \backslash G(F) / K_{v}\right)$ by

$$
\chi_{\pi}(\mathrm{f})=\operatorname{tr}(\pi(\mathrm{f}))
$$

where we fix a measure on $G(F)$ so that the volume of $K_{v}$ is one.

Definition 6.4. - We define the spherical parameter associated to $\pi$ to be the unique Langlands parameter

$$
\operatorname{Sat}(\pi): W_{F} \rightarrow{ }^{L} G
$$

such that $\chi_{\mathrm{Sat}(\pi)}=\chi_{\pi}$ under the Satake isomorphism

$$
C_{c}\left(K_{v} \backslash G(F) / K_{v}\right) \simeq \mathrm{R} .
$$


As explained in Lemma A.13, in the case $F=\mathbb{F}_{q}((\mathrm{t}))$, the isomorphism (6.3) can be deduced from 0.2 under the sheaf-function dictionary. We come back to the notation as in $\S 4$, in particular $k=\overline{\mathbb{F}}_{q}$. Let $\bar{\mu} \in \mathbb{X}_{\bullet}(T)_{I}$. By abuse of notation, we denote the corresponding Schubert variety in $\mathcal{F} \ell_{v} \otimes k$ by $\mathcal{F} \ell_{v \bar{\mu}} \otimes k$. If $\bar{\mu}$ is defined $\mathbb{F}_{q}$, i.e., $\bar{\mu} \in\left(\mathbb{X}_{\bullet}(T)_{I}\right)^{\sigma}$, then $\mathcal{F} \ell_{v \bar{\mu}} \otimes k$ is also defined over $\mathbb{F}_{q}$ and we denote the corresponding Schubert variety in $\mathcal{F} \ell_{v}$ by $\mathcal{F} \ell_{v \bar{\mu}}$. In this case the intersection cohomology sheaf $\mathrm{IC}_{\bar{\mu}}$ is naturally in $\mathscr{P}_{v}^{0}$, and $\mathrm{H}^{*}\left(\mathrm{IC}_{\bar{\mu}}\right)$ is a representation of $\left(H^{\vee}\right)^{I} \rtimes_{\text {act }}$ alg $\operatorname{Gal}\left(k / \mathbb{F}_{q}\right)$. When restricted to $\left(H^{\vee}\right)^{I}$, it is the highest representation $W_{\bar{\mu}}$. By abuse of notation, this algebraic representation of $\left(H^{\vee}\right)^{I} \rtimes_{\text {actalg }} \operatorname{Gal}\left(k / \mathbb{F}_{q}\right)$ is still denoted by $W_{\bar{\mu}}$. Let $A_{\bar{\mu}} \in C_{c}\left(K_{v} \backslash G(F) / K_{v}\right)$ be the associated function under Grothendieck's sheaf-function dictionary. Combining (6.1) and (6.2), we have

$$
\operatorname{tr}\left(\pi\left(A_{\mu}\right)\right)=\operatorname{tr}\left(\operatorname{Sat}(\pi)(\Phi), W_{\bar{\mu}}\right)
$$

\section{Applications to the nearby cycles on certain Shimura varieties}

One of the main motivations of this work is to calculate the nearby cycles for certain unitary Shimura varieties. This is achieved by the so-called Rapoport-Zink-Pappas local models.

Let $F / \mathbb{Q}$ be a quadratic imaginary field; we fix an embedding $F \subset \mathbb{C}$. Let $(W, \phi)$ be a Hermitian space over $F / \mathbb{Q}$, of dimension $n=\operatorname{dim} W \geq 3$. Let $G=\mathrm{GU}(W, \phi)$ be the group of unitary similitudes defined by

$$
G(R)=\left\{g \in \mathrm{GL}_{F}\left(W \otimes_{\mathbb{Q}} R\right) \mid \phi(g v, g w)=c(\mathrm{~g}) \phi(v, w), c(\mathrm{~g}) \in R^{\times}\right\} .
$$

Assume that $\left(W_{\mathbb{R}}, \phi_{\mathbb{R}}\right) \simeq\left(\mathbb{C}^{n}, H\right)$, where $H$ is the standard Hermitian matrix on $\mathbb{C}^{n}$ of signature $(r, s)$, i.e., $H=\operatorname{diag}\left\{(-1)^{(\mathrm{s})}, 1^{(\mathrm{r})}\right\}$ is the diagonal matrix with -1 repeated at the first $s$ places and 1 repeated at the remaining $r$ places ${ }^{(5)}$. Without loss of generality, we can assume that $s \leq r$. Let $h: \operatorname{Res}_{\mathbb{C} / \mathbb{R}} \mathbb{G}_{m} \rightarrow G_{\mathbb{R}}$ be the homomorphism given by $h(\mathrm{z})=\operatorname{diag}\left\{z^{(\mathrm{s})}, \bar{z}^{(\mathrm{r})}\right\}$. Let $K \subset G\left(\mathbb{A}_{f}\right)$ be an open compact subgroup, small enough (i.e., $K$ is contained in some principal congruence subgroup for some $N \geq 3$ ). Then associated to the data $(G,\{h\}, K)$, one can define a Shimura variety $\operatorname{Sh}(G, K)$ over a number field $E$, where $E=\mathbb{Q}$ if $r=s$, and $E=F$ if $r \neq s$. Let us recall that $h$ also determines a conjugacy class of one parameter subgroups of $G_{\mathbb{C}}$ (the Shimura cocharacter), defined over $E$. In our case, $G_{\mathbb{C}} \simeq \mathrm{GL}_{n} \times \mathbb{G}_{m}$ and the one parameter subgroups are conjugate to $\mu_{r, s}(\mathrm{z})=\left(\operatorname{diag}\left\{z^{(\mathrm{s})}, 1^{(\mathrm{r})}\right\}, z\right)$.

Let us fix a prime $p>2$ and assume that $F / \mathbb{Q}$ is ramified at $p$. We denote $F_{p}$ (resp. $E_{p}$ ) the completion of $F$ (resp. $E$ ) at the unique place over $p$. In addition, we assume that $(W, \phi)$ is a split Hermitian form at $p$. In other words, $(W, \phi)_{\mathbb{Q}_{p}} \simeq\left(F_{p}^{n}, J\right)$, where $J$ is the split Hermitian matrix on $F_{p}^{n}$ with all its anti-diagonal entries 1, and 0 elsewhere. Observe that this assumption automatically holds if $n$ is odd. Then $G_{\mathbb{Q}_{p}}$ is quasi-split. We will assume that $K=K_{p} K^{p} \subset G\left(\mathbb{Q}_{p}\right) G\left(\mathbb{A}_{f}^{p}\right)$ and $K_{p}$ is a special parahoric of $G_{\mathbb{Q}_{p}}$, which is automatically very special in the sense of (6.1). Let us make this more concretely.

(5) The corresponding Hermitian form is $H(z, w)=\underline{\bar{z}}^{t} H \underline{w}$ for $\underline{z}, \underline{w} \in \mathbb{C}^{n}$. 
Let $\pi$ be a uniformizer of $F_{p}$ so that $\pi^{2}=a p$ with $a$ a Teichmüller lifting of $\mathbb{F}_{p}^{\times}$. Let $\left\{e_{1}, \ldots, e_{n}\right\}$ be a basis of $F_{p}^{n}$ so that $\phi\left(e_{i}, e_{j}\right)$ is given by $J$. Let

$$
\Lambda_{i}=\operatorname{Span}_{\vartheta_{F_{p}}}\left\{\pi^{-1} e_{1}, \ldots, \pi^{-1} e_{i}, e_{i+1}, \ldots, e_{n}\right\} .
$$

If $n=2 m+1$, we consider two integral models for $G_{\mathbb{Q}_{p}}$,

$$
\underline{G}_{v_{0}}=\left\{g \in G, g \Lambda_{0}=\Lambda_{0}\right\}, \quad \underline{G}_{v_{1}}=\left\{g \in G, g \Lambda_{m}=\Lambda_{m}\right\} .
$$

If $n=2 m$, we consider the integral model

$$
\underline{G}_{v}=\left\{g \in G, g \Lambda_{m}=\Lambda_{m}\right\} .
$$

As explained in [23, Section 1.2], these $\underline{G}_{v}$ are special parahoric group schemes and essentially all special parahoric group schemes of $G$ are conjugate to these ones.

Let $K_{p}=\underline{G}_{v}\left(\mathbb{Z}_{p}\right)$. In this case, the Shimura variety $\operatorname{Sh}(G, K)$ has a well-defined model over $\vartheta_{E_{p}}$, as in [23]. Let us denote the integral model by $\mathrm{Sh}_{K_{p}}$. In addition, there is the socalled local model diagram

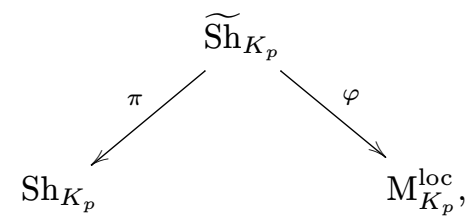

where the scheme $\mathrm{M}_{K_{p}}^{\text {loc }}$, which is called the local model of $\mathrm{Sh}_{K_{p}}$, is projective over $\theta_{E_{p}}$ with an action of $\underline{G}_{v} \otimes_{\mathbb{Z}_{p}} \theta_{E_{p}}$, and is étale locally isomorphic to $\mathrm{Sh}_{K_{p}}$. In addition, $\pi: \widetilde{\operatorname{Sh}}_{K_{p}} \rightarrow \operatorname{Sh}_{K_{p}}$ is a $\underline{G}_{v} \otimes_{\mathbb{Z}_{p}} \theta_{E_{p}}$-torsor, and $\varphi: \widetilde{\operatorname{Sh}}_{K_{p}} \rightarrow \mathrm{M}_{K_{p}}^{\text {loc }} \underline{G}_{v} \otimes_{\mathbb{Z}_{p}} \theta_{E_{p}}$-equivariant and is formally smooth (cf. [23] for details).

We are interested in the nearby cycle $\Psi_{\mathrm{Sh}_{K_{p}}}\left(\mathbb{Q}_{\ell}\right)$, which is an $\ell$-adic complex on $\mathrm{Sh}_{K_{p}} \otimes_{\vartheta_{E_{p}}} \overline{\mathbb{F}}_{p}$, on which $\Gamma=\operatorname{Gal}\left(\overline{\mathbb{Q}}_{p} / E\right)$ acts continuously, compatibly with the action of $\Gamma$ on $\mathrm{M}_{K_{p}}^{\text {loc }} \otimes \overline{\mathbb{F}}_{p}$ through $\Gamma \rightarrow \operatorname{Gal}\left(\overline{\mathbb{F}}_{p} / \mathbb{F}_{p}\right)$. From the local model diagram, we have

$$
\pi^{*} \Psi_{\mathrm{Sh}_{K_{p}}}\left(\mathbb{Q}_{\ell}\right) \simeq \varphi^{*} \Psi_{\mathrm{M}_{K_{p}}^{\mathrm{loc}}}\left(\mathbb{Q}_{\ell}\right) .
$$

Therefore, it is essentially enough to determine $\Psi_{\mathrm{M}_{K_{p}}^{\text {loc }}}\left(\mathbb{Q}_{\ell}\right)$. For this purpose, we need to recall the geometry of $\mathrm{M}_{K_{p}}^{\text {loc }}$.

First, let $F^{\prime}=\mathbb{F}_{p}((\mathrm{u}))$ be a ramified quadratic extension of $\mathbb{F}_{p}((\mathrm{t}))$ with $u^{2}=a t$, where $a \in \mathbb{F}_{p}^{\times}$as before. Let $W^{\prime}=F^{\prime} e_{1}+\cdots+F^{\prime} e_{n}$ and $\phi^{\prime}$ be a split Hermitian form on $W^{\prime}$ given by $\phi^{\prime}\left(e_{i}, e_{n+1-j}\right)=\delta_{i j}$. Let $G^{\prime}$ be the corresponding unitary similitude group over $\mathbb{F}_{p}((\mathrm{t}))$. The parahoric group scheme $\underline{G}_{v}$ of $G$ over $\mathbb{Z}_{p}$ has an obvious counterpart $\underline{G}_{v}^{\prime}$ over $\mathbb{F}_{p}[[t]]$. Namely, consider

$$
\Lambda_{i}^{\prime}=\operatorname{Span}_{\vartheta_{F^{\prime}}}\left\{\pi^{-1} e_{1}, \ldots, \pi^{-1} e_{i}, e_{i+1}, \ldots, e_{n}\right\} .
$$

If $\underline{G}_{v}=G_{\mathbb{Q}_{p}} \cap \operatorname{Aut}\left(\Lambda_{i}\right)$, then $\underline{G}_{v}^{\prime}=G^{\prime} \cap \operatorname{Aut}\left(\Lambda_{i}^{\prime}\right)$. Observe that there is an isomorphism $\underline{G}_{v} \otimes \mathbb{F}_{p} \simeq \underline{G}_{v}^{\prime} \otimes \mathbb{F}_{p}$ (given by the obvious identification of $(W, \phi)_{\mathbb{F}_{p}} \simeq\left(W^{\prime}, \phi^{\prime}\right)_{\mathbb{F}_{p}}$ ). In addition, the Shimura cocharacter $\mu_{r, s}$ makes sense as a cocharacter of $G^{\prime} \otimes\left(\mathbb{F}_{p}((\mathrm{t}))\right)^{s}$. Let $\mathcal{F} \ell_{v}=L G^{\prime} / L^{+} \underline{G}_{v}^{\prime}$ be the associated affine flag variety considered before.

Now, we give the description of $\mathrm{M}_{K_{p}}^{\text {loc }}$. The following statements can be extracted from $[23,25,24]$. 
Proposition 7.1. - (i) The generic fiber of $\mathrm{M}_{K_{p}}^{\mathrm{loc}}$ is isomorphic to $\mathscr{P}_{\mu_{r, s}}$, where $\mathscr{P}_{\mu_{r, s}}$ is the variety of maximal parabolic subgroups of $G_{E_{p}}$ of the type given by $\mu_{r, s}$.

(ii) The special fiber is isomorphic to $\mathcal{F} \ell_{v \bar{\mu}_{r, s}}$ in an equivariant way. More precisely, the $L^{+} \underline{G}_{v}^{\prime}$-action on $\mathcal{F} \ell_{v \bar{\mu}_{r, s}}$ factors through an action of $\underline{G}_{v}^{\prime} \otimes \mathbb{F}_{p}$, and there is an isomorphism

$$
\mathrm{M}_{K_{p}}^{\mathrm{loc}} \simeq \mathcal{F} \ell_{v \bar{\mu}_{r, s}}
$$

intertwining the $\underline{G}_{v} \otimes \mathbb{F}_{p}$ action on the left and this $\underline{G}_{v}^{\prime} \otimes \mathbb{F}_{p}$-action on the right.

(iii) The generic point of the special fiber $\mathrm{M}_{K_{p}}^{\mathrm{loc}} \otimes \mathbb{F}_{p}$ is smooth in $\mathrm{M}_{K_{p}}^{\text {loc }}$.

Having described the geometry of $\mathrm{M}_{K_{p}}^{\text {loc }}$, let us state the main theorem of this section. First, let $\mathscr{P}_{v}$ be either

1. the category of $L^{+} \underline{G}_{v}^{\prime}$-equivariant Weil perverse sheaves on $\mathcal{F} \ell_{v}$, constant along each $L^{+} \underline{G}_{v}^{\prime}$-orbit; or

2. the category of $L^{+} \underline{G}_{v}^{\prime} \otimes \overline{\mathbb{F}}_{p}$-equivariant perverse sheaves on $\mathcal{F} \ell_{v} \otimes \overline{\mathbb{F}}_{p}$.

Likewise, we understand $\mathrm{IC}_{\bar{\mu}_{r, s}}$ either as a pure perverse sheaf of weight zero, or just a geometric perverse sheaf. By Theorem 0.1, we have:

1. if $n=2 m+1$ is odd,

$$
\mathscr{R} \varnothing: \operatorname{Rep}\left(\mathrm{GO}_{2 m+1}\right) \simeq \mathscr{P}_{v}
$$

2. if $n=2 m$ is even,

$$
\mathscr{R} \varnothing: \operatorname{Rep}\left(\mathrm{GSp}_{2 m}\right) \simeq \mathscr{P}_{v}
$$

Next, let $V$ be the standard representation of $\mathrm{GL}_{n}$ and $V_{r, s}=\wedge^{s} V$ to be its $s$ th wedge power. We extend $V_{r, s}$ to a representation of $\mathrm{GL}_{n} \times \mathbb{G}_{m}$, on which $\mathbb{G}_{m}$ acts via the homotheties.

TheOREM 7.2. - Regard $V_{r, s}$ as a representation of $\mathrm{GO}_{n} \subset \mathrm{GL}_{n} \times \mathbb{G}_{m}$ if $n$ is odd, or of $\mathrm{GSp}_{n} \subset \mathrm{GL}_{n} \times \mathbb{G}_{m}$ if $n$ is even by restriction. Then:

1. Denote by $\Psi^{\text {geom }}$ the underlying complex of sheaves of $\Psi_{\mathrm{M}_{K_{p}}^{\text {loc }}}[r s]\left(\frac{r s}{2}\right)$ on $\mathrm{M}_{K_{p}}^{\text {loc }} \otimes \overline{\mathbb{F}}_{p}$. Then

$$
\Psi^{\text {geom }} \simeq \mathscr{R} \phi\left(V_{r, s}\right) \simeq \begin{cases}\mathrm{IC}_{\bar{\mu}_{r, s}} & n \text { odd }, \\ \sum_{s^{\prime} \geq 0, s-s^{\prime} \in 2 \mathbb{Z}_{\geq 0}} \mathrm{IC}_{\bar{\mu}_{n-s^{\prime}, s^{\prime}}} & \text { n even } .\end{cases}
$$

2. Let $r \neq s$. Then the action of the inertial subgroup $I \subset \Gamma$ on $\Psi_{\mathrm{M}_{K p}^{\mathrm{loc}}}$ is trivial so that $\Psi_{\mathrm{M}_{K_{p}}^{\text {loc }}}$ admits a structure as a Weil sheaf on $\mathcal{F} \ell_{v}$. In addition, as Weil sheaves,

$$
\Psi_{\mathrm{M}_{K_{p}}^{\text {loc }}}[r s]\left(\frac{r s}{2}\right) \simeq \mathscr{R} \phi\left(V_{r, s}\right)
$$

3. Let $r=s=m$ where $n=2 m$. By (1)

$$
\Psi^{\text {geom }} \simeq \sum_{m^{\prime} \geq 0, m-m^{\prime} \in 2 \mathbb{Z} \geq 0} \mathrm{IC}_{\bar{\mu}_{n-m^{\prime}, m^{\prime}}} .
$$

The action of the inertial subgroup $I$ on $\Psi_{\mathrm{M}_{K_{p}}^{\text {loc }}}$ factors through $I \rightarrow \operatorname{Gal}\left(F_{p} / \mathbb{Q}_{p}\right) \simeq \mathbb{Z} / 2$. In addition, the action of $\mathbb{Z} / 2$ on $\mathrm{IC}_{\bar{\mu}_{n-m^{\prime}, m^{\prime}}}$ is trivial if $4 \mid m-m^{\prime}$ and is through the non-trivial character if $4 \nmid m-m^{\prime}$. As Weil sheaves,

$$
\left(\Psi_{\mathrm{M}_{K_{p}}^{\mathrm{Ioc}}}\right)^{I}[r s]\left(\frac{r s}{2}\right) \simeq \sum_{m^{\prime} \geq 0, m-m^{\prime} \in 4 \mathbb{Z}_{\geq 0}} \mathrm{IC}_{\bar{\mu}_{n-m^{\prime}, m^{\prime}}},
$$


where $\left(\Psi_{\mathrm{M}_{K_{p}}^{\mathrm{loc}}}^{\mathrm{loc}}\right)^{I}$ denotes the inertial invariants of $\Psi_{\mathrm{M}_{K_{p}}^{\mathrm{loc}}}$.

Proof. - Observe that $\Psi^{\text {geom }}$ is an object in $\mathscr{P}_{v}$. This follows from Proposition 7.1 (ii). Observe that $\mathrm{IC}_{\bar{\mu}_{r, s}}$ is a direct summand of $\Psi^{\text {geom }}$. This follows from the fact that $\mathrm{M}_{K_{p}}^{\text {loc }}$ is flat over $\vartheta_{E_{p}}$ with special fiber isomorphic to $\mathcal{F} \ell_{v \bar{\mu}_{r, s}}$.

The partially flag variety $\mathscr{P}_{\mu_{r, s}}$ is a Schubert variety in the affine Grassmanian of $G_{E_{p}}$ over $E_{p}$, (see (A.2)). Therefore, $\mathrm{H}^{*}\left(\mathscr{P}_{\mu_{r, s}}\right)$ is a natural representation of $\mathrm{GL}_{n} \times \mathbb{G}_{m}$ (the dual group of $G_{E_{p}}$ ), which is indeed just $V_{r, s}$. Since nearby cycles commute with proper pushforward, we have

$$
V_{r, s} \simeq \mathrm{H}^{*}\left(\mathscr{D}_{\mu_{r, s}}\right) \simeq \mathrm{H}^{*}\left(\Psi^{\text {geom }}\right) .
$$

Part (1) of the theorem would follow if we can show that this isomorphism is an isomorphism of $\mathrm{GO}_{n}$ or $\mathrm{GSp}_{n}$-modules. This is indeed the case, and can be shown using the constructions in [24]. In fact, as a further application of the main results of this paper, we will prove the corresponding Part (1) for all ramified groups in loc. cit. Here for the ramified unitary groups, we give a more direct (and easier) argument, without showing that (7.3) is an isomorphism of $\mathrm{GO}_{n}$ or $\mathrm{GSp}_{n}$-modules. (However, we do need the existence of this natural isomorphism as graded vector spaces.) In fact, we will deduce the theorem from the following description of the restriction of $V_{r, s}$ as a representation of $\left(\mathrm{GL}_{n} \times \mathbb{G}_{m}\right)^{I}$. Note that if $n$ is odd, then $V_{r, s}$ remains irreducible as a representation of $\mathrm{GO}_{n}=\left(\mathrm{GL}_{n} \times \mathbb{G}_{m}\right)^{I}$, denoted by $W_{\bar{\mu}_{r, s}}$. If $n$ is even, the symplectic form induces a surjective map $V_{r, s} \rightarrow V_{r+2, s-2}$ and the kernel is the irreducible representation of $\mathrm{GSp}_{n}=\left(\mathrm{GL}_{n} \times \mathbb{G}_{m}\right)^{I}$ of highest weight $\bar{\mu}_{r, s}$, denoted by $W_{\bar{\mu}_{r, s}}$. Finally, if $(r, s)=(m, m), V_{m, m}$ canonically extends to a representation of $\left(\mathrm{GL}_{n} \times \mathbb{G}_{m}\right) \rtimes I$ (e.g., see Corollary A.11).

Lemma 7.3. - (i) As $\left(\mathrm{GL}_{n} \times \mathbb{G}_{m}\right)^{I}$-modules,

$$
V_{r, s} \simeq \begin{cases}W_{\bar{\mu}_{r, s}} & n \text { odd }, \\ \sum_{s^{\prime} \geq 0, s-s^{\prime} \in 2 \mathbb{Z}_{\geq 0}} W_{\bar{\mu}_{n-s^{\prime}, s^{\prime}}} & n \text { even } .\end{cases}
$$

(ii) The representation $V_{m, m}$ of $\left(\mathrm{GL}_{n} \times \mathbb{G}_{m}\right) \rtimes I$, when restricted to $\mathrm{GSp}_{n} \rtimes I=\mathrm{GSp}_{n} \times I$, decomposes as

$$
V_{m, m}=\sum_{m^{\prime} \geq 0, m-m^{\prime} \in 2 \mathbb{Z}_{\geq 0}} W_{\bar{\mu}_{n-m^{\prime}, m^{\prime}}} \otimes \chi_{m^{\prime}},
$$

where $\chi_{m^{\prime}}$ is the trivial character of $\operatorname{Gal}\left(F_{p} / \mathbb{Q}_{p}\right)$ if $4 \mid m-m^{\prime}$, and is the non-trivial character if $4 \nmid m-m^{\prime}$.

Proof. - (i) is clear and we prove (ii). Clearly, there are some characters $\chi_{m^{\prime}}$ of $\operatorname{Gal}\left(F_{p} / \mathbb{Q}_{p}\right)$ such that the decomposition (7.4) holds. We need to identify these characters.

First, it is clear that $\chi_{m}=1$. This is because the lowest weight space of $V_{\bar{\mu}_{m, m}}$ is the same as the lowest weight space of $V_{m, m}$, which in turn is the same as $\mathrm{H}^{0}\left(\mathscr{P}_{\mu_{m, m}}\right)$ as $I$-modules. But the action of $I$ on $\mathrm{H}^{0}\left(\mathscr{P}_{\mu_{m, m}}\right)$ is trivial. This shows that $\chi_{m}=1$.

Now pick up $g \in I$ whose projection to $\operatorname{Gal}\left(F_{p} / \mathbb{Q}_{p}\right) \simeq \mathbb{Z} / 2$ is non-trivial. To identify other $\chi_{m^{\prime}}$, let us write the weight lattice of $\mathrm{GL}_{n} \times \mathbb{G}_{m}$ in a standard way to be $\mathbb{X}^{\bullet}=\bigoplus \mathbb{Z} \varepsilon_{i} \bigoplus \mathbb{Z} \varepsilon$ and the set of simple roots to be $\left\{\varepsilon_{i}-\varepsilon_{i+1}, 1 \leq i \leq n-1\right\}$. Then 
the action of $g$ on $\mathbb{X} \bullet$ will send $\varepsilon_{i}$ to $-\varepsilon_{n+1-i}$ and $\varepsilon$ to $\varepsilon+\varepsilon_{1}+\cdots+\varepsilon_{n}$. The weight lattice of $\mathrm{GSp}_{n}$ is $\mathbb{X} \bullet /\left\{\varepsilon_{i}+\varepsilon_{n+1-i}=0, i=1, \ldots, m\right\}$.

Let $\left\{v_{1}, \ldots, v_{n}\right\}$ be a standard basis of $V$ so that $v_{i}$ is a weight vector of $\mathrm{GL}_{n}$ of weight $\varepsilon_{i}$ as usual. Then a basis of $V_{m, m}$ is given by $\left\{v_{i_{1}} \wedge \cdots \wedge v_{i_{m}} \mid 1 \leq i_{1}<\cdots<i_{m} \leq n\right\}$. We divide this set of basis into two subsets $A$ and $B$. A base vector $v_{i_{1}} \wedge \cdots \wedge v_{i_{m}}$ belongs to the subset $A$ if $\{i, n+1-i\} \nsubseteq\left\{i_{1}, \ldots, i_{m}\right\}$ for any $1 \leq i \leq m$. All remaining base vectors belong to $B$. It is clear that $\operatorname{Span}\{v \mid v \in A\} \subset V_{\bar{\mu}_{m, m}}$ and therefore, the action of $g$ fixes each $v \in A$ since $\chi_{m}=1$. On the other hand, it is easy to see from the description of the action of $g$ on $\mathbb{X}^{\bullet}$, that for $v \in B, g v$ will be a multiple of some $w \in B, w \neq v$. From this, we deduce that for any $t$ in the maximal torus of $\mathrm{GSp}_{n}$,

$$
\operatorname{tr}\left(g t, V_{m, m}\right)=\varepsilon(\mathrm{t}) \sum \varepsilon_{1}(\mathrm{t})^{ \pm 1} \cdots \varepsilon_{m}(\mathrm{t})^{ \pm 1} .
$$

On the other hand, according to (7.4), we have

$$
\operatorname{tr}\left(g t, V_{m, m}\right)=\sum_{m^{\prime} \geq 0, m-m^{\prime} \in 2 \mathbb{Z}_{\geq 0}} \chi_{m^{\prime}}(\mathrm{g}) \operatorname{ch}\left(W_{\bar{\mu}_{n-m^{\prime}, m^{\prime}}}\right)(\mathrm{t}),
$$

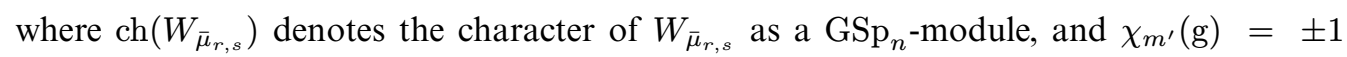
according to whether $\chi_{m^{\prime}}$ is trivial or not. Now it is easy to see that the above two identities force $\chi_{m^{\prime}}=1$ if $4 \mid m-m^{\prime}$ and $\chi_{m^{\prime}} \neq 1$ if $4 \nmid m-m^{\prime}$. Indeed, let $T$ be an indeterminant and write

$$
\varepsilon\left(1+\varepsilon_{1} T\right) \cdots\left(1+\varepsilon_{m} T\right)\left(1+\varepsilon_{1}^{-1} T\right) \cdots\left(1+\varepsilon_{m}^{-1} T\right)=\sum a_{k} T^{k},
$$

then $a_{m+i}=a_{m-i}$ and $\operatorname{ch}\left(W_{\bar{\mu}_{r, s}}\right)=a_{s}-a_{s-2}=a_{r}-a_{r+2}$. Put $T=\sqrt{-1}$, the left hand side becomes $(\sqrt{-1})^{m} \varepsilon\left(\varepsilon_{1}+\varepsilon_{1}^{-1}\right) \cdots\left(\varepsilon_{m}+\varepsilon_{m}^{-1}\right)$, which is exactly (7.5), and the right hand side is $(\sqrt{-1})^{m}\left(a_{m}-2 a_{m-2}+2 a_{m-4}-\cdots\right)$. The lemma is proved.

Now we prove the theorem. We first assume that $n$ is odd. As $V_{r, s}$ remains irreducible as a representation of $\mathrm{GO}_{n}$. Therefore, $\mathcal{R} \&\left(V_{r, s}\right) \simeq \mathrm{IC}_{\bar{\mu}_{r, s}}$ and we have

$$
\operatorname{dim} V_{r, s}=\operatorname{dim} \mathrm{H}^{*}\left(\mathrm{IC}_{\bar{\mu}_{r, s}}\right) \leq \operatorname{dim} \mathrm{H}^{*}\left(\Psi^{\text {geom }}\right)=\operatorname{dim} \mathrm{H}^{*}\left(\mathscr{D}_{\mu_{r, s}}\right)=\operatorname{dim} V_{r, s} .
$$

Therefore, $\Psi^{\text {geom }}=\mathrm{IC}_{\bar{\mu}_{r, s}}$. As $\mathrm{IC}_{\bar{\mu}_{r, s}}$ is irreducible, the inertial group $I$ acts on $\Psi_{\mathrm{M}_{K_{p}}^{\text {loc }}}$ via some character. Observe that the action of $I$ on $\mathrm{H}^{0}\left(\Psi_{\mathrm{M}_{K_{p}}^{\text {loc }}}\right) \simeq \mathrm{H}^{0}\left(\mathscr{P}_{\mu_{r, s}}\right) \simeq \mathbb{Q}_{\ell}$ is via the same character, again due to the fact that nearby cycles commute with proper push-forward. Therefore the action of $I$ on $\Psi_{\mathrm{M}_{K_{p}}^{\text {loc }}}$ is trivial. Therefore, $\Psi_{\mathrm{M}_{K_{p}}^{\mathrm{loc}}}[r s]\left(\frac{r s}{2}\right) \simeq \mathrm{IC}_{\bar{\mu}_{r, s}} \otimes \mathscr{L}$ for some rank one local system $\mathcal{L}$ on Spec $\mathbb{F}_{p}$. By comparing the action of Frobenius on $\mathrm{H}^{*}\left(\mathscr{D}_{\mu_{r, s}}\right)$ and on $\mathrm{H}^{*}\left(\mathrm{IC}_{\bar{\mu}_{r, s}}\right)$, we obtain the theorem in this case.

Now we assume that $n$ is even. Recall that under the (ramified) geometric Satake isomorphism, the cohomological grading corresponds to the grading by $2 \rho: \mathbb{G}_{m} \rightarrow \mathrm{GSp}_{n} \subset \mathrm{GL}_{n} \times \mathbb{G}_{m}$. Therefore, (7.3) is an isomorphism of the representations of $2 \rho\left(\mathbb{G}_{m}\right) \subset \mathrm{GSp}_{n}$. We claim that this already implies Part (1) of the theorem. Indeed, for a representation $V$ of $\mathrm{GSp}_{n}$, we denote $V(\mathrm{i})$ to be the eigenspace of $2 \rho$ of eigenvalue $i$. Let us write

$$
\Psi^{\text {geom }}=\sum_{m^{\prime} \geq 0, m-m^{\prime} \in 2 \mathbb{Z}_{\leq 0}} c_{m^{\prime}} \mathrm{IC}_{\bar{\mu}_{n-m^{\prime}, m^{\prime}}}
$$


we need to show that $c_{m^{\prime}}=1$. First as in Lemma 2.6, $c_{s}=1$ by Proposition 7.1 (iii). Next, we show that $c_{s-2}=1$. Observe that the gradings on $\mathrm{H}^{*}\left(\mathrm{IC}_{\bar{\mu}_{r, s}}\right)$ range from $r s$ to $-r s$. From

$$
\begin{aligned}
& \operatorname{dim} \mathrm{H}^{(s-2)(r+2)}\left(\Psi^{\text {geom }}\right)=c_{s-2}+\operatorname{dim} \mathrm{H}^{(s-2)(r+2)}\left(\mathrm{IC}_{\bar{\mu}_{r, s}}\right), \\
& \operatorname{dim} V_{r, s}((s-2)(r+2))=1+\operatorname{dim}\left(W_{\bar{\mu}_{r, s}}((s-2)(r+2))\right),
\end{aligned}
$$

we conclude that $c_{s-2}=1$. Now by induction, $c_{m^{\prime}}=1$ for all $m^{\prime} \geq 0, m-m^{\prime} \in 2 \mathbb{Z}_{\geq 0}$. This shows that

$$
\Psi^{\text {geom }} \simeq \mathscr{R} \&\left(V_{r, s}\right) .
$$

Next, we determine the action of the inertial group $I$ on $\Psi_{\mathrm{M}_{K_{p}}^{\text {loc }}}$. First, assume that $r \neq s$. To show that the action of $I$ on $\Psi_{\mathrm{M}_{K_{p}}^{\text {loc }}}$ is trivial, we again observe that $I$ acts on each irreducible direct summand of $\Psi_{\mathrm{M}_{K_{p}}^{\text {loc }}}$ via a certain character. On the other hand, the group $G_{E_{p}}$ is split. Therefore, the action of $I$ on $\mathrm{H}^{*}\left(\mathscr{P}_{\mu_{r, s}}\right)$ is trivial, and therefore is trivial on $\Psi_{\mathrm{M}_{K_{p}}^{\text {loc }}}$. Again, comparing the action of the Frobenius, we conclude the result in this case.

Finally, let us assume that $r=s=m$, where $n=2 m$. Then $E_{p}=\mathbb{Q}_{p}$ and $G_{\mathbb{Q}_{p}}$ is not split. In addition the action of $I$ on $\mathrm{H}^{*}\left(\mathscr{P}_{\mu_{m, m}}\right)$ is not trivial. Indeed, as $\mathscr{P}_{\mu_{m, m}}$ is defined over $\mathbb{Q}_{p}$, according to the appendix, $V_{\mu_{m, m}}=\mathrm{H}^{*}\left(\mathscr{P}_{\mu_{m, m}}\right)$ is a natural representation of ${ }^{L} G^{\text {geom }}=\left(\mathrm{GL}_{n} \times \mathbb{G}_{m}\right) \rtimes_{\text {actgeom }} \operatorname{Gal}\left(\overline{\mathbb{Q}}_{p} / \mathbb{Q}_{p}\right)$, so that the natural action of $\operatorname{Gal}\left(\overline{\mathbb{Q}}_{p} / \mathbb{Q}_{p}\right)$ on $\mathrm{H}^{*}\left(\mathscr{P}_{\mu_{m, m}}\right)$ is given by the restriction of this representation to $\mathrm{Gal}\left(\overline{\mathbb{Q}}_{p} / \mathbb{Q}_{p}\right)$. This semidirect product $\left(\mathrm{GL}_{n} \times \mathbb{G}_{m}\right) \rtimes_{\text {actgeom }} \mathrm{Gal}\left(\overline{\mathbb{Q}}_{p} / \mathbb{Q}_{p}\right)$ is not the Langlands dual group ${ }^{L} G_{\mathbb{Q}_{p}}^{\text {alg }}$ of $G_{\mathbb{Q}_{p}}$. But if we form both semi-product using $I \subset \operatorname{Gal}\left(\overline{\mathbb{Q}}_{p} / \mathbb{Q}_{p}\right)$, they become the same because the cyclotomic character is trivial on $I$. On the other hand, since $G$ is split over $F_{p}$, the action of $I$ factors through $I \rightarrow \operatorname{Gal}\left(F_{p} / \mathbb{Q}_{p}\right) \simeq \mathbb{Z} / 2$. We know that

$$
\Psi^{\text {geom }}=\sum_{m^{\prime} \geq 0, m-m^{\prime} \in 2 \mathbb{Z} \geq 0} \mathrm{IC}_{\bar{\mu}_{n-m^{\prime}, m^{\prime}}} .
$$

As argued in the case $r \neq s$, the action of $I$ on $\Psi_{\mathrm{M}_{K_{p}}^{\text {loc }}}$ also factors through $I \rightarrow \operatorname{Gal}\left(F_{p} / \mathbb{Q}_{p}\right)$. Assume that the action of $I$ on $\mathrm{IC}_{\bar{\mu}_{n-m^{\prime}, m^{\prime}}}$ is through the character $\chi_{m^{\prime}}^{\prime}$. We need to show that $\chi_{m^{\prime}}^{\prime}=\chi_{m^{\prime}}$, where $\chi_{m^{\prime}}$ is as in Lemma 7.3. Since $\mathrm{H}^{*}\left(\Psi_{\mathrm{M}_{K_{p}}^{\text {loc }}}\right) \simeq \mathrm{H}^{*}\left(\mathscr{P}_{\mu_{m, m}}\right) \simeq V_{m, m}$ as $\left(2 \rho\left(\mathbb{G}_{m}\right) \times I\right)$-modules, by taking the $I$-invariants, we obtain that

$$
\sum_{\chi_{m^{\prime}}^{\prime}=1} \mathrm{H}^{*}\left(\mathrm{IC}_{\bar{\mu}_{n-m^{\prime}, m^{\prime}}}\right)=\sum_{\chi_{m^{\prime}}=1} W_{\bar{\mu}_{n-m^{\prime}, m^{\prime}}} .
$$

Again, as argued before by considering the gradings, it is easy to see that this forces $\chi_{m^{\prime}}^{\prime}=\chi_{m^{\prime}}$. Finally, by comparing the action of Frobenius on $\mathrm{H}^{*}\left(\Psi_{\mathrm{M}_{K_{p}}^{\mathrm{loc}}}^{I}\right)$ and on $\mathrm{H}^{*}\left(\mathscr{P}_{\mu_{m, m}}\right)^{I}$, we conclude the theorem.

Combining Theorem 7.2 and Theorem 5.1, it is not hard to obtain the explicit formula of the trace of Frobenius of $\Psi_{\mathrm{M}_{K_{p}}^{\mathrm{loc}}}$, which will be the input of the Langlands-Kottwitz method of calculating the local Zeta function of the Shimura varieties. Instead of writing down the explicit formula, let us characterize this function in terms of its trace on "unramified" representations of $G(F)$ (which clearly determines this function uniquely). The characterization verifies a conjecture of Haines and Kottwitz in this case. 
Proposition 7.4. - Let $z_{r, s}$ be the function on $G^{\prime}(F)$ associated to $\Psi_{\mathrm{M}_{K_{p}}^{\text {loc }}}^{I}$ under the Grothendieck sheaf-function dictionary, and let $V_{r, s}$ be the representation of ${ }^{L} G_{E_{p}}^{\text {alg }}$ attached to $\mu_{r, s} \in \mathbb{X}_{\bullet}(T)$ as above (or in Corollary A.11). For $\pi$ an "unramified" representation of $G^{\prime}(F)$, with the Langlands parameter $\operatorname{Sat}(\pi)$ as defined in (6.4), we have

$$
\operatorname{tr}\left(\pi\left(z_{r, s}\right)\right)=\operatorname{tr}\left(\operatorname{Sat}(\pi)(\Phi), V_{r, s}^{I}\right) .
$$

The proof is a direct consequence of Theorem 7.2 and (6.4).

REMARK 7.5. - In the Langlands-Kottwitz methods of calculating the Zeta factors of Shimura varieties, one needs some mysterious test functions $z_{\mu}$ to be put into the trace formula. Assuming the Local Langlands, Haines and Kottwitz give a conjectural characterization of this test function $z_{\mu}$ in the general setting (i.e., arbitrary group and arbitrary level structure). In the special case when the group is quasi-split and the level structure is special parahoric, in which case the Langlands parameters is clear (as in Section 6), their characterization is reduced to the above proposition. Therefore, this proposition is the first example of their conjecture in the case when the group is ramified at $p$. In [24], we will show that the same characterization holds for arbitrary (tamely ramified) quasi-split groups with special parahoric level structure.

Finally, let us make Theorem 7.2 more explicit for some special cases.

Corollary 7.6. - Let $(r, s)=(n-1,1)$. Then the inertial group acts on $\Psi_{\mathrm{Sh}_{K_{p}}}$ trivially. In addition, as Weil sheaves, $\Psi_{\mathrm{Sh}_{K_{p}}} \simeq \mathbb{Q}_{\ell}$. In particular, for every $x \in \operatorname{Sh}_{K_{p}}\left(\mathbb{F}_{p^{n}}\right)$, $\operatorname{tr}\left(\operatorname{Frob}_{x}, \Psi_{K_{p}}\right)=1$.

Proof. - The first statement follows from Theorem 7.2 and the local model diagram. We need to show that $\mathrm{IC}_{\bar{\mu}_{n-1,1}} \simeq \overline{\mathbb{Q}}_{\ell}[n-1]\left(\frac{n-1}{2}\right)$. However, according to Theorem 5.1, we know that $\mathrm{IC}_{\bar{\mu}_{n-1,1}}[1-n]\left(\frac{1-n}{2}\right)$ is a sheaf (for the standard $t$-structure) rather than a complex, with each stalk isomorphic to $\overline{\mathbb{Q}}_{\ell}$. As $\operatorname{IC}_{\bar{\mu}_{n-1,1}}[1-n]\left(\frac{1-n}{2}\right)$ is indecomposable as object in $D\left(\mathcal{F} \ell_{v}\right)$, this forces $\operatorname{IC}_{\bar{\mu}_{n-1,1}}[1-n]\left(\frac{1-n}{2}\right) \simeq \overline{\mathbb{Q}}_{\ell}$. Now, since $\pi^{*} \Psi_{K_{p}} \simeq \varphi^{*} \operatorname{IC}_{\bar{\mu}_{n-1,1}}[1-n]\left(\frac{1-n}{2}\right)$ and $\pi$ has geometrically connected fibers, the corollary follows.

Remark 7.7. - Concerning the part of the Frobenius trace, this corollary has been proven in [17, 23, 25]. Indeed, for the case $n$ is odd, and the special parahoric is $\underline{G}_{v_{0}}$, this is a main result of [17]. In this case, $\mathrm{Sh}_{K_{p}}$ is not semi-stable. For the case $n$ is odd and the parahoric is $\underline{G}_{v_{1}}$, it is shown in [25] that $\mathrm{Sh}_{K_{p}}$ is smooth. For the case $n$ is even, it is shown in [23] that $\mathrm{Sh}_{K_{p}}$ is smooth.

Next, we consider the case $(r, s)=(2,2)$. Recall that the local model diagram can be written as a morphism

$$
\mathrm{Sh}_{K_{p}} \rightarrow\left[\underline{G}_{v} \backslash \mathrm{M}_{K_{p}}^{\mathrm{loc}}\right]
$$

where $\left[\underline{G}_{v} \backslash \mathrm{M}_{K_{p}}^{\text {loc }}\right]$ denotes the stack quotient. Therefore, the Schubert stratification on $\mathrm{M}_{K_{p}}^{\text {loc }} \otimes \overline{\mathbb{F}}_{p}$ induces a stratification on $\mathrm{Sh}_{K_{p}} \otimes \overline{\mathbb{F}}_{p}$, called the Kottwitz-Rapoport (KR) stratification. In the case $(r, s)=(2,2)$, the stratification has two strata $\mathrm{Sh}_{K_{p}, b}$ and $\mathrm{Sh}_{K_{p}, s}$. The smaller one $\mathrm{Sh}_{K_{p}, s}$ is zero-dimensional. Similarly to the previous case, we have 
COROLlary 7.8. - Let $(r, s)=(2,2)$. Then $\Psi_{\mathrm{Sh}_{K_{p}}}=\Psi_{\mathrm{Sh}_{K_{p}}}^{1}+\Psi_{\mathrm{Sh}_{K_{p}}}^{2}$. The inertial action on $\Psi_{\mathrm{Sh}_{K_{p}}}^{1}$ is trivial and as Weil sheaves, $\Psi_{\mathrm{Sh}_{K_{p}}}^{1} \simeq \mathbb{Q}_{\ell}$. The vanishing cycle $\Phi_{\mathrm{Sh}_{K_{p}}}\left(\mathbb{Q}_{\ell}\right) \simeq \Psi_{K_{p}}^{2}$. When we forget the action of $\operatorname{Gal}\left(\overline{\mathbb{Q}}_{p} / \mathbb{Q}_{p}\right), \Psi_{\mathrm{Sh}_{K_{p}}}^{2}=\sum_{x \in \operatorname{Sh}_{K_{p}, s}} \delta_{x}[-4]$, where $\delta_{x}$ is the delta sheaf supported at $x$. In addition, the inertial action on $\delta_{x}$ factors through a non-trivial quadratic character. In particular, for every $x \in \operatorname{Sh}_{K_{p}}\left(\mathbb{F}_{p^{n}}\right), \operatorname{tr}\left(\operatorname{Frob}_{x}, \Psi_{\mathrm{Sh}_{K_{p}}}^{I}\right)=1$.

\section{Appendix}

\section{Construction of the full Langlands dual group via the geometric Satake correspondence by Timo Richarz \& Xinwen Zhu}

In the main body of the paper, we considered a reductive group $G$ over $F=k((\mathrm{t}))$ ( $k$ algebraically closed), split over a tamely ramified extension, and recovered $\left(H^{\vee}\right)^{\operatorname{Gal}\left(F^{s} / F\right)}$ by the Tannakian formalism from a certain category of perverse sheaves associated to $G$, where $H^{\vee}$ is the dual group of $G$, on which $\operatorname{Gal}\left(F^{s} / F\right)$ acts via pinned automorphisms. In this appendix, we take a different point of view to recover the full Langlands dual group ${ }^{L} G=H^{\vee} \rtimes \operatorname{Gal}\left(F^{s} / F\right)$ of $G$ by the Tannakian formalism. The construction is easy but we cannot find it in the literature. Most proofs will be omitted or rather sketched since they are very simple.

Let us begin with a review of certain general nonsense of Tannakian formalism. A similar discussion appears in [14, Appendix 2]. Let $(\mathscr{C}, \omega)$ be a neutralized Tannakian category over a field $E$ of characteristic zero with fiber functor $\omega$. We define a monoidal category $\operatorname{Aut}^{\otimes}(\mathscr{C}, \omega)$ as follows: objects are pairs $(\sigma, \alpha)$, where $\sigma: \mathscr{C} \rightarrow \mathscr{C}$ is a tensor automorphism and $\alpha: \omega \circ \sigma \simeq \omega$ is a natural isomorphism of tensor functors; morphisms between $(\sigma, \alpha)$ and $\left(\sigma^{\prime}, \alpha^{\prime}\right)$ are natural tensor isomorphisms between $\sigma$ and $\sigma^{\prime}$ that are compatible with $\alpha, \alpha^{\prime}$ in an obvious way. The monoidal structure is given by compositions. Since $\omega$ is faithful, $\operatorname{Aut}^{\otimes}(\mathscr{C}, \omega)$ is (equivalent to) a set, and in fact is a group. For example, $(\sigma, \alpha)=$ id means that there is an isomorphism $\varepsilon: \sigma \simeq$ id of tensor functors such that $\omega \varepsilon=\alpha$ (such $\varepsilon$ will be unique).

Let $H=$ Aut $_{\mathscr{C}}^{\otimes} \omega$, the Tannakian group defined by $(\mathscr{C}, \omega)$. Let $\operatorname{Aut}(H)$ be the group of automorphisms of $H$ and $\operatorname{Out}(H)$ be the group of outer automorphisms of $H$.

Lemma A.1. - There is a canonical action of $\operatorname{Aut}^{\otimes}(\mathscr{C}, \omega)$ on $H$ by automorphisms. In addition, the map $\operatorname{Aut}^{\otimes}(\mathscr{C}, \omega) \rightarrow \operatorname{Aut}(H)$ induces $\left[\operatorname{Aut}^{\otimes}(\mathscr{C})\right] \rightarrow \operatorname{Out}(H)$, where $\left[\operatorname{Aut}^{\otimes}(\mathscr{C})\right]$ is the group of isomorphism classes of tensor automorphisms of $\mathscr{C}$.

The action of $(\sigma, \alpha)$ on $H$ is given as follows. Let $R$ be an $E$-algebra and $h: \omega_{R} \simeq \omega_{R}$ be an $R$-point of $H$. Then $(\sigma, \alpha) h$ is the following composition

$$
\omega_{R} \stackrel{\alpha}{\leftarrow} \omega_{R} \circ \sigma \stackrel{h \circ \text { id }}{\rightarrow} \omega_{R} \circ \sigma \stackrel{\alpha}{\rightarrow} \omega_{R} .
$$

Remark A.2. - As is shown [14], $\operatorname{Aut}^{\otimes}(\mathscr{C}, \omega)$ can be upgraded into a fppf sheaf on the category of affine schemes over $E$, and as $f p p f$ sheaves $\operatorname{Aut}^{\otimes}(\mathscr{C}, \omega) \simeq \operatorname{Aut}(H)$. We do not need this fact. 
Let $\Gamma$ be an abstract group. We define an action of $\Gamma$ on $(\mathscr{C}, \omega)$ to be a group homomorphism act $: \Gamma \rightarrow \operatorname{Aut}^{\otimes}(\mathscr{C}, \omega)$. Assume that $\Gamma$ acts on $(\mathscr{C}, \omega)$. We can then define $\mathscr{C}^{\Gamma}$, the category of $\Gamma$-equivariant objects in $\mathscr{C}$ as follows: objects are $\left(X,\left\{c_{\gamma}\right\}_{\gamma \in \Gamma}\right)$, where $X$ is an object in $\mathscr{C}$ and $c_{\gamma}: \operatorname{act}_{\gamma}(X) \simeq X$ is an isomorphism, satisfying the natural cocycle condition, i.e., $c_{\gamma^{\prime}} \circ \operatorname{act}_{\gamma^{\prime}}\left(c_{\gamma}\right)=c_{\gamma^{\prime} \gamma}$; the morphisms between $\left(X,\left\{c_{\gamma}\right\}_{\gamma \in \Gamma}\right)$ and $\left(X^{\prime},\left\{c_{\gamma}^{\prime}\right\}_{\gamma \in \Gamma}\right)$ are morphisms between $X$ and $X^{\prime}$, compatible with $c_{\gamma}, c_{\gamma}^{\prime}$ in an obvious way.

Lemma A.3. - Let $\Gamma$ be a group acting on $(\mathscr{C}, \omega)$. Then the category $\mathscr{C}^{\Gamma}$ is a neutral Tannakian category, with fiber functor $\omega$. In addition, if $\Gamma$ is a finite group, regarded as an algebraic E-group, then the Tannakian group $\mathrm{Aut}_{\mathscr{C}^{\Gamma}}^{\otimes} \omega$ is canonically isomorphic to $H \rtimes \Gamma$.

Proof. - The monoidal structure on $\mathscr{C}^{\Gamma}$ is defined as

$$
\left(X,\left\{c_{\gamma}\right\}_{\gamma \in \Gamma}\right) \otimes\left(X^{\prime},\left\{c_{\gamma}^{\prime}\right\}_{\gamma \in \Gamma}\right)=\left(X^{\prime \prime},\left\{c_{\gamma}^{\prime \prime}\right\}_{\gamma \in \Gamma}\right),
$$

where $X^{\prime \prime}=X \otimes X^{\prime}$ and $c_{\gamma}^{\prime \prime}: \operatorname{act}_{\gamma}\left(X^{\prime \prime}\right) \rightarrow X^{\prime \prime}$ is the composition

$$
\operatorname{act}_{\gamma}\left(X \otimes X^{\prime}\right) \simeq \operatorname{act}_{\gamma}(X) \otimes \operatorname{act}_{\gamma}\left(X^{\prime}\right) \stackrel{c_{\gamma} \otimes c_{\gamma}^{\prime}}{\longrightarrow} X \otimes X^{\prime} .
$$

This gives $\mathscr{C}^{\Gamma}$ the structure of a Tannakian category. Now assume that $\Gamma$ is finite, and hence $H \rtimes \Gamma$ is an affine group scheme. By [7, Prop. 2.8], it is enough to show that,

$$
\operatorname{Rep}(H)^{\Gamma} \stackrel{\simeq}{\longrightarrow} \operatorname{Rep}(H \rtimes \Gamma)
$$

as tensor categories compatible with the forgetful functors. Let $\left((V, \rho),\left\{c_{\gamma}\right\}_{\gamma \in \Gamma}\right) \in \operatorname{Rep}(H)^{\Gamma}$. Then we define $(V, \tilde{\rho}) \in \operatorname{Rep}(H \rtimes \Gamma)$, for any $k$-algebra $R(h, \gamma) \in(H \rtimes \Gamma)(R)$, by

$$
(h, \gamma) \longmapsto \rho(\mathrm{h}) \circ \alpha_{g, R}(V) \circ \omega_{R} \circ c_{\gamma}^{-1} \in \mathrm{GL}(V \otimes R),
$$

where $\alpha_{g, R}: \omega_{R} \circ \sigma_{g} \simeq \omega_{R}$ is induced by the action of $\Gamma$ as above. Using the cocycle relation one checks that this is indeed a representation, and that the map defines the desired equivalence.

Remark A.4. - If $\Gamma$ is not finite, then the category $\left(\mathscr{C}^{\Gamma}, \omega\right)$ is still Tannakian, but $\tilde{H}=$ Aut $_{\mathscr{C}^{\Gamma}}^{\otimes} \omega$ is no longer $H \rtimes \Gamma$ since the latter cannot be regarded as an affine group scheme (sometimes $\tilde{H}$ is called the algebraic envelop of $H \rtimes \Gamma$ ). However, there is always a group homomorphism

$$
H(E) \rtimes \Gamma \rightarrow \tilde{H}(E) .
$$

Although this is not an isomorphism in general, we may still regard $\omega(X)$ for $X \in \mathscr{C}^{\Gamma}$ as a representation of $H(E) \rtimes \Gamma$.

Now, we assume that $G$ is a connected reductive group over any field $k$. We switch the notation to use $G^{\vee}$ to denote the reductive group over $E=\overline{\mathbb{Q}}_{\ell}$ dual to $G$ in the sense of Langlands, i.e., the root datum of $G^{\vee}$ is dual to the root datum of $G_{k^{s}}$. Up to the choice of a pinning $\left(G^{\vee}, B^{\vee}, T^{\vee}, X^{\vee}\right)$ of $G^{\vee}$, we have an action of $\Gamma_{k}=\operatorname{Gal}\left(k^{s} / k\right)$ on $G^{\vee}$ via

$$
\Gamma_{k} \rightarrow \operatorname{Out}\left(G_{k^{s}}\right) \simeq \operatorname{Out}\left(G^{\vee}\right) \simeq \operatorname{Aut}\left(G^{\vee}, B^{\vee}, T^{\vee}, X^{\vee}\right) \subset \operatorname{Aut}\left(G^{\vee}\right) .
$$

Then the Langlands dual group ${ }^{L} G$ is defined to be $G^{\vee} \rtimes \Gamma_{k}$. Our goal is to recover this group via the above Tannakian formalism. 
Let $L^{+} G$ be the jet group of $G \otimes k[[t]]$ and $L G$ be the loop group of $G \otimes k((\mathrm{t}))$. Recall that by definition, for every $k$-algebra $R, L^{+} G(R)=G(R[[t]])$ and $L G(R)=G(R((\mathrm{t})))$. Let

$$
\operatorname{Gr}_{G}=L G / L^{+} G
$$

be the affine Grassmannian of $G$ over $k$. Let $\mathrm{Gr}_{G} \otimes k^{s}$ be its base change to the separable closure of $k$. From [31, Lemma 3.3], formation of the affine Grassmannian commutes with étale base change, we have $\mathrm{Gr}_{G} \otimes k^{s} \simeq \mathrm{Gr}_{G_{k^{s}}}$. Since $G_{k^{s}}$ is split, we can consider the usual Satake category Sat on $\mathrm{Gr}_{G_{k} s}$, i.e., the category of $\left(L^{+} G \otimes k^{s}\right)$-equivariant perverse sheaves on $\operatorname{Gr}_{G} \otimes k^{s}$, which is equivalent to $\operatorname{Rep}\left(G_{\overline{\mathbb{Q}}_{\ell}}^{\vee}\right)$ via the geometric Satake correspondence of [19]. Note that the Galois group $\Gamma_{k}$ acts on $\mathrm{Gr}_{G} \otimes k^{s}$. For $\gamma \in \Gamma_{k}$, the pullback functor $\gamma^{*}: D\left(\mathrm{Gr}_{G} \otimes k^{s}\right) \rightarrow D\left(\mathrm{Gr}_{G} \otimes k^{s}\right)$ clearly restricts to a functor $\gamma^{*}:$ Sat $\rightarrow$ Sat. In addition, there is a canonical isomorphism $\alpha_{\gamma}: \mathrm{H}^{*}\left(\gamma^{*} \mathcal{F}\right) \simeq \mathrm{H}^{*}(\mathcal{F})$.

Lemma A.5. - The assignment $\gamma \mapsto\left(\gamma^{*}, \alpha_{\gamma}\right)$ defines an action of $\Gamma_{k}$ on $\left(\mathrm{Sat}, \mathrm{H}^{*}\right)$.

According to Lemma A.1, there is a canonical action of $\Gamma_{k}$ on $G^{\vee}$, denoted by act ${ }^{\text {geom }}$. And we can form

$$
{ }^{L} G^{\text {geom }}:=G^{\vee} \rtimes_{\text {actgeom }} \Gamma_{k},
$$

which we will call the geometric Langlands dual group.

Now, our goal is to understand the relation between ${ }^{L} G^{\text {geom }}$ and the usual Langlands dual group ${ }^{L} G$. Recall that in Section 4, we explained that once we choose an ample line bundle on $\mathrm{Gr}_{G}$, the geometric Satake isomorphism provides $G^{\vee}$ with a canonical pinning $\left(G^{\vee}, B^{\vee}, T^{\vee}, X^{\vee}\right)$. Therefore, there is an action of $\Gamma_{k}$ on $G^{\vee}$ via (A.1), denoted by act ${ }^{\text {alg }}$. Then we can form the usual Langlands dual group by ${ }^{L} G^{\text {alg }}=G^{\vee} \rtimes_{\text {actalg }} \Gamma_{k}$. It turns out that the difference between act ${ }^{\text {geom }}$ and act ${ }^{\text {alg }}$ can be described explicitly.

Let

$$
\operatorname{cycl}: \Gamma_{k} \rightarrow \mathbb{Z}_{\ell}^{\times}
$$

be the cyclotomic character of $\Gamma_{k}$ defined by the action of $\Gamma_{k}$ on the $\ell^{\infty}$-roots of unity of $k^{s}$. Let $G_{\mathrm{ad}}^{\vee}$ be the adjoint group of $G^{\vee}$. Let $\rho$ be the half sum of positive coroots of $G^{\vee}$, which gives rise to a one-parameter group $\rho: \mathbb{G}_{m} \rightarrow G_{\mathrm{ad}}^{\vee}$. We define a map

$$
\chi: \Gamma_{k} \stackrel{\text { cycl }}{\rightarrow} \mathbb{Z}_{\ell}^{\times} \stackrel{\rho}{\rightarrow} G_{\mathrm{ad}}^{\vee}\left(\overline{\mathbb{Q}}_{\ell}\right),
$$

which gives a map $\operatorname{Ad}_{\chi}: \Gamma_{k} \rightarrow \operatorname{Aut}\left(G^{\vee}\right)$ to the inner automorphism of $G^{\vee}$.

Proposition A.6. - Let $\left(G^{\vee}, B^{\vee}, T^{\vee}, X^{\vee}\right)$ be the pinning defined by the geometric Satake isomorphism as in $\S 4$. We have act ${ }^{\text {geom }}=\operatorname{act}^{\text {alg }} \circ \mathrm{Ad}_{\chi}$.

Proof. - Observe that the action of $\Gamma_{k}$ preserves the cohomological grading. In addition, $\Gamma_{k}$ acts on $X^{\vee}$ through cycl since $X^{\vee}$ is the Chern class of the chosen ample line bundle on $\operatorname{Gr}_{G}$. Therefore, $\operatorname{Ad}_{\chi^{-1}}$ ○ act ${ }^{\text {geom }}$ preserves $\left(G^{\vee}, B^{\vee}, T^{\vee}, X^{\vee}\right)$. But since $\operatorname{Ad}_{\chi^{-1}} \circ$ act ${ }^{\text {geom }}$ and act ${ }^{\text {alg }}$ act on the based root datum $(\mathbb{X} \bullet(T), \Delta)$ by the same way, these two actions must coincide. 
REMARK A.7. - (i) An interesting corollary of the above proposition is that the act ${ }^{\text {geom }}$ of $\Gamma_{k}$ on $G^{\vee}$ only depends on the quasi-split form of $G$, since the same is true for act ${ }^{\text {alg }}$.

(ii) The existence of these two actions act ${ }^{\text {geom }}$ and act ${ }^{\text {alg }}$ is a geometric source of the two natural normalizations for the Satake parameters. In addition, these two actions are also parallel to the notions of $C$-algebraic and $L$-algebraic, as recently introduced by Buzzard and Gee [5].

COROLlary A.8. - We have ${ }^{L} G^{\text {alg }} \stackrel{\simeq}{\longrightarrow} G^{\text {geom }}$ given by

$$
(g, \gamma) \mapsto\left(\operatorname{Ad}_{\chi\left(\gamma^{-1}\right)}(\mathrm{g}), \gamma\right)
$$

Following [3, 2.6], we define the notation of the algebraic representations of ${ }^{L} G^{\text {alg }}$ as follows. For every $k^{\prime} \subset k^{s}$ finite over $k$ such that $G_{k^{\prime}}$ is split, one can form the "finite form" of Langlands dual group $G^{\vee} \rtimes_{\text {actalg }} \operatorname{Gal}\left(k^{\prime} / k\right)$, which can be regarded as an algebraic group over $\overline{\mathbb{Q}}_{\ell}$. Therefore, we may consider ${ }^{L} G^{\text {alg }}=\varliminf_{\lim } G^{\vee} \rtimes_{\text {act alg }} \operatorname{Gal}\left(k^{\prime} / k\right)$ as a pro-algebraic group over $\overline{\mathbb{Q}}_{\ell}$, Then it makes sense to talk about the category of algebraic representations $\operatorname{Rep}\left({ }^{L} G^{\text {alg }}\right)$ of ${ }^{L} G^{\text {alg }}$, which is the inductive limit of $\operatorname{Rep}\left(G^{\vee} \rtimes_{\text {actalg }} \operatorname{Gal}\left(k^{\prime} / k\right)\right)$.

Now, we consider certain categories of perverse sheaves. First, according to the above discussions, we call the action of $\Gamma_{k}$ on Sat via $\gamma \mapsto\left(\gamma^{*}, \alpha_{\gamma}\right)$ as in Lemma A.5 the geometric action. We can also define an algebraic action of $\Gamma_{k}$ on Sat as $\gamma \mapsto\left(\gamma^{*}, \chi(\gamma)^{-1} \alpha_{\gamma} \chi(\gamma)\right)$. Clearly, there is a canonical isomorphism between $\mathrm{Sat}^{\Gamma_{k}, \text { geom }}$ and $\mathrm{Sat}^{\Gamma_{k} \text {,alg }}$ sending $\left(\mathcal{F},\left\{c_{\gamma}\right\}_{\gamma \in \Gamma_{k}}\right)$ to $\left(\mathcal{F},\left\{c_{\gamma}\right\}_{\gamma \in \Gamma_{k}}\right)$. The reason we distinguish them is due to the following observation: since the algebraic action of $\Gamma_{k}$ on $G^{\vee}$ factors through $\operatorname{Gal}\left(k^{\prime} / k\right)$ if $G_{k^{\prime}}$ is split, the algebraic action of $\Gamma_{k}$ on Sat also factors through $\operatorname{Gal}\left(k^{\prime} / k\right)$, and therefore it makes sense to talk about $\mathrm{Sat}^{\mathrm{Gal}\left(k^{\prime} / k\right), \text { alg }}$, which is naturally a full category of $\mathrm{Sat}^{\Gamma_{k}, \text { alg }}$. In addition,

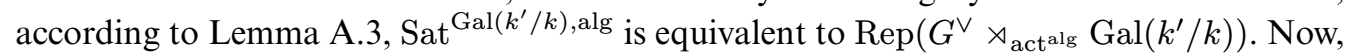
let $\mathrm{Sat}^{\Gamma_{k}, \text { alg, } f} \subset \mathrm{Sat}^{\Gamma_{k}, \text { alg }}$ be the full subcategory, which is the union of all $\mathrm{Sat}^{\operatorname{Gal}\left(k^{\prime} / k\right), \text { alg }}$. We obtain that

$$
\mathrm{H}^{*}: \mathrm{Sat}^{\Gamma_{k}, \text { alg }, f} \simeq \operatorname{Rep}\left({ }^{L} G^{\text {alg }}\right) .
$$

Our next goal then is to identify $\mathrm{Sat}^{\Gamma_{k}, \text { alg, } f}$ as a subcategory of $\mathrm{Sat}^{\Gamma_{k}, \text { geom }}$.

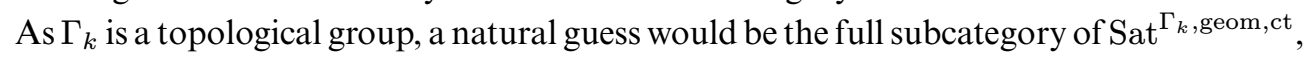
consisting of objects on which $\Gamma_{k}$ acts continuously. Equivalent, let $\mathscr{P}_{L^{+} G}\left(\mathrm{Gr}_{G}\right)$ be the category of perverse sheaves on $\mathrm{Gr}_{G}$. Then the pullback functor to $\mathrm{Gr}_{G} \otimes k^{s}$ induces $\mathscr{P}_{L^{+} G}\left(\mathrm{Gr}_{G}\right) \simeq \mathrm{Sat}^{\Gamma_{k}, \text { geom }, \mathrm{ct}}$. However, it is not the case that $\mathrm{Sat}^{\Gamma_{k}, \text { geom }, \mathrm{ct}}=\mathrm{Sat}^{\Gamma_{k}, \text { alg }, f}$.

Definition A.1. - (i) Let $X$ be a smooth variety over $k$, we define a constant sheaf to be a direct sum of $\left(\overline{\mathbb{Q}}_{\ell}[1]\left(\frac{1}{2}\right)\right)^{\otimes \operatorname{dim} X}$.

(ii) We define $\mathscr{P}_{L^{+}{ }_{G}}^{f}\left(\mathrm{Gr}_{G}\right)$ to be the full subcategory of $\mathscr{P}_{L^{+} G}\left(\mathrm{Gr}_{G}\right)$, consisting of those sheaves $\mathcal{F}$, such that there exists some $k^{\prime} \supset k$ such that $\mathcal{F} \otimes k^{\prime}$ is constant along each $\left(L^{+} G \otimes k^{\prime}\right)$-orbit.

REMARK A.9. - (i) The toy model is when $G=\{e\}$ is the trivial group. Then $\mathscr{P}_{L^{+}}{ }_{G}\left(\mathrm{Gr}_{G}\right)$ is the category $\Gamma_{k}$-Mod of continuous representations of $\Gamma_{k}$ while $\mathscr{P}_{L^{+} G}^{f}\left(\operatorname{Gr}_{G}\right)$ is the subcategory $\Gamma_{k}$-Mod ${ }^{f}$ consisting of representations of finite quotients of $\Gamma_{k}$. In particular, if $k$ is a finite field, we can identify the latter as the category of semi-simple $\Gamma_{k}$-modules, pure of weight zero. 
(ii) Observe that every object in $\mathscr{P}_{L^{+} G}\left(\mathrm{Gr}_{G}\right)$ is of the form $\oplus_{i} \mathrm{IC}_{i} \otimes \mathscr{L}_{i}$, where $\mathrm{IC}_{i}$ is an intersection cohomology sheaf on $\mathrm{Gr}_{G}$, and $\mathscr{L}_{i}$ is a representation of $\Gamma_{k}$. Therefore, $\mathscr{F} \in \mathscr{P}_{L^{+} G}^{f}\left(\mathrm{Gr}_{G}\right)$ if and only if all $\mathscr{L}_{i} \in \Gamma_{k}$ - $\operatorname{Mod}^{f}$. In particular, if $k=\mathbb{F}_{q}$ is a finite field, the category $\mathscr{D}_{L^{+} G}^{f}\left(\mathrm{Gr}_{G}\right)$ is equivalent to the category of semi-simple $L^{+} G$-equivariant perverse sheaves on $\mathrm{Gr}_{G}$, pure of weight zero.

Proposition A.10. - Under the canonical isomorphism $\mathrm{Sat}^{\Gamma_{k}, \text { geom }}=\mathrm{Sat}^{\Gamma_{k}, \text { alg }}$, we have the identification

$$
\mathscr{P}_{L^{+} G}^{f}\left(\mathrm{Gr}_{G}\right)=\mathrm{Sat}^{\Gamma_{k}, \mathrm{alg}, f} .
$$

Therefore, we obtain an equivalence of tensor categories

$$
\mathrm{H}^{*}: \mathscr{D}_{L^{+} G}^{f}\left(\operatorname{Gr}_{G}\right) \rightarrow \operatorname{Rep}\left({ }^{L} G^{\mathrm{alg}}\right) .
$$

Proof. - Let $k^{\prime} \supset k$ such that $G_{k^{\prime}}$ splits. We denote $\mathscr{P}_{L^{+} G, k^{\prime}}\left(\mathrm{Gr}_{G}\right)$ the full subcategory of $\mathscr{P}_{L^{+} G}^{f}\left(\mathrm{Gr}_{G}\right)$ consisting of those $\mathcal{F}$ such that $\mathscr{F} \otimes k^{\prime}$ is constant along each $\left(L^{+} G \otimes k^{\prime}\right)$-orbit. Then under

$$
\mathscr{P}_{L^{+} G}\left(\mathrm{Gr}_{G}\right) \rightarrow \mathrm{Sat}^{\Gamma_{k}, \text { geom }} \simeq \mathrm{Sat}^{\Gamma_{k}, \text { alg }},
$$

$\mathscr{P}_{L^{+} G, k^{\prime}}\left(\mathrm{Gr}_{G}\right)$ maps to Sat ${ }^{\mathrm{Gal}\left(k^{\prime} / k\right), \text { alg }}$. To see this, one reduces to the case when $G$ is split over $k$ and $k^{\prime}=k$. In this case $\mathscr{P}_{L^{+} G, k}\left(\mathrm{Gr}_{G}\right) \simeq$ Sat and this statement is clear.

For every $\mathcal{F} \in \mathscr{D}_{L^{+} G}^{f}\left(\mathrm{Gr}_{G}\right)$, let us describe $\mathrm{H}^{*}(\mathcal{F})$ as a representation of ${ }^{L} G^{\text {alg }}$ more explicitly. First, as an object in Sat ${ }^{\Gamma_{k}}$,geom , it is a natural representation of ${ }^{L} G^{\text {geom }}$, on which $G^{\vee}$ acts via the usual geometric Satake isomorphism, and $\Gamma_{k}$ acts via the natural Galois action. Then the action of ${ }^{L} G^{\text {alg }}$ is via (A.8).

In particular, if $\overline{\mathrm{Gr}}_{\mu}$ is a Schubert variety in $\mathrm{Gr}_{G}$ defined over $k$ (i.e., the conjugacy class of the one-parameter subgroup determined by $\mu$ is defined over $k$ ), then $\mathrm{IC}_{\overline{\mathrm{Gr}}_{\mu}}$ is an object in $\mathscr{D}_{L^{+} G}^{f}\left(\mathrm{Gr}_{G}\right)$. Therefore, $\mathrm{H}^{*}\left(\mathrm{IC}_{\overline{\mathrm{Gr}}_{\mu}}\right)$ is a representation of $G^{\vee} \rtimes_{\text {actalg }} \operatorname{Gal}\left(k^{\prime} / k\right)$, where $k^{\prime}$ is the splitting field of $G$. We thus obtain

Corollary A.11. - Let $V_{\mu}$ be a representation of $G^{\vee}$ of highest weight $\mu$. If the conjugacy class of the one parameter subgroup $\mu: \mathbb{G}_{m} \rightarrow G_{k^{s}}$ is defined over $k$, then $V_{\mu}$ can be extended canonically to a representation of $G^{\vee} \rtimes_{\text {actalg }} \operatorname{Gal}\left(k^{\prime} / k\right)$, where $k^{\prime}$ is the splitting field of $G$.

Now we specialize to the case that $k=\mathbb{F}_{q}$ is a finite field, so that $G \otimes k[[t]]$ is a hyperspecial group scheme for the unramified group $G_{k((\mathrm{t}))}$. Note that ${ }^{L} G_{k(\mathrm{t}))}^{\mathrm{alg}}={ }^{L} G^{\text {alg }}$. We therefore obtain the geometric Satake isomorphism for unramified groups.

Theorem A.12. - Let $\mathscr{P}_{L^{+} G}^{f}\left(G r_{G}\right)$ be the category of semi-simple, $L^{+} G$-equivariant perverse sheaves on $\mathrm{Gr}_{G}$, pure of weight zero. Then we have an equivalence of tensor categories

$$
\mathrm{H}^{*}: \mathscr{P}_{L^{+} G}^{f}\left(\mathrm{Gr}_{G}\right) \simeq \operatorname{Rep}\left({ }^{L} G^{\text {alg }}\right) .
$$

4 e SÉRIE - TOME $48-2015$ - No 2 
Let $\sigma$ be the Frobenius element in $\Gamma_{k}$. Denote by $\mathscr{H}_{G}$ the Grothendieck ring of $\mathscr{P}_{L^{+} G}^{f}\left(\mathrm{Gr}_{G}\right)$, tensored with $\overline{\mathbb{Q}}_{\ell}$, and by $\mathscr{R}_{L}$ the algebra associated to $\operatorname{Rep}\left({ }^{L} G^{\text {alg }}\right)$. Theorem A.12 gives an isomorphism of algebras

$$
\Phi: \mathscr{H}_{G} \rightarrow \mathscr{R}_{L} \text {. }
$$

Let $\mathrm{H}_{G}$ be the spherical Hecke algebra of compactly supported bi- $G\left(\mathbb{F}_{q}[[t]]\right)$-invariant functions. Let $\mathrm{R}_{L_{G}}$ be the algebra of $\overline{\mathbb{Q}}_{\ell}$-valued functions on $\left(G^{\vee} \times \sigma\right)_{s s}$, generated by the characters of elements in $\operatorname{Rep}\left({ }^{L} G^{\text {alg }}\right)$. Here, $\left(G^{\vee} \times \sigma\right)_{s s}$ is the set of semi-simple elements in $G^{\vee} \times \sigma$, as defined in [3, Section 6]. We have a surjective map of algebras $\operatorname{Tr}: \mathcal{H}_{G} \rightarrow \mathrm{H}_{G}$ (resp. Ch: $\mathscr{R}_{L_{G}} \rightarrow \mathrm{R}_{L_{G}}$ ) given by the trace of Frobenius (resp. by sending a representation to its character).

Lemma A.13. - The isomorphism (A.3) induces a unique isomorphism

$$
\phi: \mathrm{H}_{G} \rightarrow \mathrm{R}_{L_{G}}
$$

such that $\mathrm{Ch} \circ \Phi=\phi \circ \operatorname{Tr}$.

Proof. - Uniqueness is clear and we show the existence. For an object $X$ in either category, we denote by $[X]$ its class in the Grothendieck ring. Then it is easy to see that the kernel of the map $\operatorname{Tr}: \mathscr{R}_{L_{G}} \rightarrow \mathrm{R}_{L_{G}}$ is the ideal generated by elements of the form $[V \otimes \psi]-\psi(\sigma)[V]$, where $V \in \operatorname{Rep}\left({ }^{L} G^{\text {alg }}\right)$ and $\psi: \Gamma_{k} \rightarrow \overline{\mathbb{Q}}_{\ell}^{\times}$is a character of $\Gamma_{k}$ factoring through a finite quotient. On the other hand, the kernel of the map $\mathrm{Ch}: \mathscr{H}_{G} \rightarrow \mathrm{H}_{G}$ is the ideal generated by elements of the form $[\mathcal{F} \otimes \mathscr{L}]-\operatorname{tr}(\sigma, \mathscr{L})[\mathcal{F}]$, where $\mathcal{F} \in \mathscr{P}_{L^{+} G}^{f}\left(\operatorname{Gr}_{G}\right)$, and $\mathscr{L}$ is a rank one local system on $\mathrm{Spec}_{q}$, pure of weight zero. But it is clear that these two ideals match under $\Phi$. The lemma follows.

Recall that by $[3,6.7]$ the classical Satake isomorphism also gives an isomorphism of algebras $\tilde{\phi}: \mathrm{H}_{G} \simeq \mathrm{R}_{L_{G}}$. By tracking back the construction of geometric Satake correspondence and the classical Satake isomorphism, one can show that $\phi=\tilde{\phi}$. Indeed, if $G=T$ is a toruse, this is clear. For general $G$, one observes that the fiber functor decomposes as a direct sum of weight functors [19, §3]: $\mathscr{P}_{L^{+} G}^{f}\left(\mathrm{Gr}_{G}\right) \rightarrow \mathscr{P}_{L^{+} T}^{f}\left(\mathrm{Gr}_{T}\right)$, and under the sheaf-function dictionary this corresponds to the constant term map CT: $\mathrm{H}_{G} \rightarrow \mathrm{H}_{T}$. Therefore, either $\phi$ or $\tilde{\phi}$ is uniquely determined by the following commutative diagram

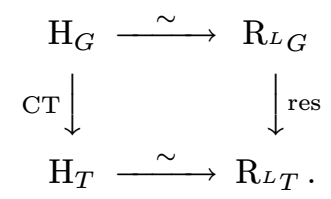

The general case follows.

\section{BIBLIOGRAPHY}

[1] J. Bernstein, V. Lunts, Equivariant sheaves and functors, Lecture Notes in Math. 1578, Springer, Berlin, 1994. 
[2] R. Bezrukavnikov, On tensor categories attached to cells in affine Weyl groups, in Representation theory of algebraic groups and quantum groups, Adv. Stud. Pure Math. 40, Math. Soc. Japan, Tokyo, 2004, 69-90.

[3] A. Borel, Automorphic L-functions, in Automorphic forms, representations and L-functions (Proc. Sympos. Pure Math., Oregon State Univ., Corvallis, Ore., 1977), Part 2, Proc. Sympos. Pure Math., XXXIII, Amer. Math. Soc., Providence, R.I., 1979, 27-61.

[4] R. K. BRYLINSKI, Limits of weight spaces, Lusztig's $q$-analogs, and fiberings of adjoint orbits, J. Amer. Math. Soc. 2 (1989), 517-533.

[5] K. Buzzard, T. Gee, The conjectural connections between automorphic representations and Galois representations, preprint arXiv:1009.0785.

[6] P. CARTiER, Representations of p-adic groups: a survey, in Automorphic forms, representations and L-functions (Proc. Sympos. Pure Math., Oregon State Univ., Corvallis, Ore., 1977), Part 1, Proc. Sympos. Pure Math., XXXIII, Amer. Math. Soc., Providence, R.I., 1979, 111-155.

[7] P. Deligne, J. Milne, Tannakian categories, in Hodge Cycles and Motives, Lecture Notes in Math. 900, 1982, 101-228.

[8] D. Gaitsgory, Construction of central elements in the affine Hecke algebra via nearby cycles, Invent. Math. 144 (2001), 253-280.

[9] D. Gaitsgory, Braiding compatibility, appendix to [2].

[10] V. A. Ginzburg, Perverse sheaves on a loop group, and Langlands duality, preprint arXiv:alg-geom/9511007.

[11] T. Haines, M. Rapoport, On parahoric subgroups, appendix to [22].

[12] T. J. Haines, S. Rostami, The Satake isomorphism for special maximal parahoric Hecke algebras, Represent. Theory 14 (2010), 264-284.

[13] T. Heines, A proof of the Kazhdan-Lusztig purity theorem via the decomposition theorem of BBD, preprint www2 math. umd.edu/ ${ }^{\mathrm{tj} h} / \mathrm{KL}$-purity1.pdf.

[14] J. Heinloth, B.-C. NGô, Z. Yun, Kloosterman sheaves for reductive groups, Ann. of Math. 177 (2013), 241-310.

[15] R. E. Kоттwitz, Points on some Shimura varieties over finite fields, J. Amer. Math. Soc. 5 (1992), 373-444.

[16] R. E. Kottwitz, Isocrystals with additional structure. II, Compositio Math. 109 (1997), 255-339.

[17] N. Krämer, Local models for ramified unitary groups, Abh. Math. Sem. Univ. Hamburg 73 (2003), 67-80.

[18] G. Lusztig, Singularities, character formulas, and a $q$-analog of weight multiplicities, in Analysis and topology on singular spaces, II, III (Luminy, 1981), Astérisque 101, Soc. Math. France, Paris, 1983, 208-229.

[19] I. Mirković, K. Vilonen, Geometric Langlands duality and representations of algebraic groups over commutative rings, Ann. of Math. 166 (2007), 95-143.

[20] D. Nadler, Perverse sheaves on real loop Grassmannians, Invent. Math. 159 (2005), $1-73$.

$4^{\mathrm{e}}$ SÉRIE - TOME $48-2015$ - No 2 
[21] G. Pappas, On the arithmetic moduli schemes of PEL Shimura varieties, J. Algebraic Geom. 9 (2000), 577-605.

[22] G. Pappas, M. Rapoport, Twisted loop groups and their affine flag varieties, $A d v$. Math. 219 (2008), 118-198.

[23] G. PAppas, M. Rapoport, Local models in the ramified case. III. Unitary groups, J. Inst. Math. Jussieu 8 (2009), 507-564.

[24] G. Pappas, X. Zhu, Local models of Shimura varieties and a conjecture of Kottwitz, Invent. Math. 194 (2013), 147-254.

[25] T. Richarz, Local models and Schubert varieties in twisted affine Grassmannians, preprint arXiv:1011.5416.

[26] R. Steinberg, Endomorphisms of linear algebraic groups, Memoirs of the American Mathematical Society 80, Amer. Math. Soc., Providence, R.I., 1968.

[27] J. Tits, Reductive groups over local fields, in Automorphic forms, representations and L-functions (Proc. Sympos. Pure Math., Oregon State Univ., Corvallis, Ore., 1977), Part 1, Proc. Sympos. Pure Math., XXXIII, Amer. Math. Soc., Providence, R.I., 1979, 29-69.

[28] J.-L. Verdier, Spécialisation de faisceaux et monodromie modérée, in Analysis and topology on singular spaces, II, III (Luminy, 1981), Astérisque 101, Soc. Math. France, Paris, 1983, 332-364.

[29] Z. Yun, X. ZHU, Integral homology of loop groups via Langlands dual groups, Represent. Theory 15 (2011), 347-369.

[30] X. ZHU, Affine Demazure modules and $T$-fixed point subschemes in the affine Grassmannian, Adv. Math. 221 (2009), 570-600.

[31] X. ZHU, On the coherence conjecture of Pappas and Rapoport, Ann. of Math. 180 (2014), 1-85.

\author{
Xinwen ZHU \\ Department of Mathematics \\ California Institute of Technology \\ 1200 E. California Blvd. \\ Pasadena, CA 91125, USA \\ E-mail: xzhu@caltech.edu
}

